\title{
Review Article \\ Recent Experimental Efforts on High-Pressure Supercritical Injection for Liquid Rockets and Their Implications
}

\author{
Bruce Chehroudi \\ Advanced Technology Consultants, Laguna Niguel, CA 92677, USA \\ Correspondence should be addressed to Bruce Chehroudi, chehroudib@aol.com
}

Received 6 March 2012; Revised 3 June 2012; Accepted 11 June 2012

Academic Editor: David Greatrix

Copyright () 2012 Bruce Chehroudi. This is an open access article distributed under the Creative Commons Attribution License, which permits unrestricted use, distribution, and reproduction in any medium, provided the original work is properly cited.

\begin{abstract}
Pressure and temperature of the liquid rocket thrust chambers into which propellants are injected have been in an ascending trajectory to gain higher specific impulse. It is quite possible then that the thermodynamic condition into which liquid propellants are injected reaches or surpasses the critical point of one or more of the injected fluids. For example, in cryogenic hydrogen/oxygen liquid rocket engines, such as Space Shuttle Main Engine (SSME) or Vulcain (Ariane 5), the injected liquid oxygen finds itself in a supercritical condition. Very little detailed information was available on the behavior of liquid jets under such a harsh environment nearly two decades ago. The author had the opportunity to be intimately involved in the evolutionary understanding of injection processes at the Air Force Research Laboratory (AFRL), spanning sub- to supercritical conditions during this period. The information included here attempts to present a coherent summary of experimental achievements pertinent to liquid rockets, focusing only on the injection of nonreacting cryogenic liquids into a high-pressure environment surpassing the critical point of at least one of the propellants. Moreover, some implications of the results acquired under such an environment are offered in the context of the liquid rocket combustion instability problem.
\end{abstract}

\section{Introduction}

In designs of chemical rocket engines, liquid fuel and oxidizer are often injected as round jets into a hot and elevatedpressure environment of the thrust chamber. The coaxial and impinging jets injectors are two of the well-established designs in liquid rocket engines (LREs). On the other hand, higher specific impulse is a major motivation for operating rocket thrust chambers at progressively higher pressures. Conditions therefore exist in which the injected liquid finds itself near or even above the thermodynamic critical point. Examples are Space Shuttle Main Engine and Vulcain (Ariane 5) with liquid $\mathrm{H}_{2}$ /liquid $\mathrm{O}_{2}$.

In such cases, major changes occur in some important and key properties of a substance as it approaches the thermodynamic critical point. For example, under thermodynamic equilibrium, the distinction between the liquid and gas phases disappears at and above the critical point and hence it is referred to as a "fluid." Also, large changes in density occur near the critical point. The constant-pressure specific heat becomes very large and surface tension vanishes at and beyond the critical point. As the ambient pressure into which a liquid jet is injected increases, the importance of the solubility of ambient gases into the injected liquid phase increases and one should consider multicomponent phase equilibrium information. For mixtures, determination of the critical conditions, called the "critical mixing temperature or pressure", is a complex process; see Bruno and Ely [1] and Lazar and Faeth [2]. For example, when a pure liquid hydrocarbon fuel drop is introduced into a nitrogen gas, a thin layer on its surface is a mixture of dissolved nitrogen and the fuel which spreads spatially in time; see Umemura [3].

Understanding the behavior of jets under supercritical conditions therefore is critical to design and modeling of the liquid rockets, in particular, cryogenic liquid rockets. For this reason, systematic research programs, both experimental and computations, have been initiated in the past 20 years to understand behaviors of jets under transcritical and supercritical conditions both with and without externally forced (acoustic) excitations. The reason the external excitation is considered stems from the combined experimental/theoretical work suggesting that interaction between 
acoustic resonance modes of the chamber and the jets could play an important role in combustion instability. The basic premise here is that when an important dynamic feature, such as the injected jet's dark-core or breakup zone, of an injector design becomes sufficiently sensitive to thermofluid parameters of its environment, it is highly likely that this could strengthen the feedback link thought to be critical in the amplification process and hence push the system into an unstable operating regime.

The purpose of this paper is to present an overview of important experimental achievements, characterizing and understanding nonreacting steady liquid jets injected into supercritical conditions, and offer some implications of these results and potential linkages to production engines. It is not the intention of this work to provide a comprehensive review of the subject, rather to present important findings reported in recent decades.

The coverage of the injectors' experimental data in this paper is divided into three parts: single liquid jets, coaxial jets, and impinging jets. However, the treatment for the impinging jets is comparatively shorter due to limited data available extending to supercritical conditions. Two sections are considered for each part. One focuses on jets injected into an environment devoid of any externally imposed acoustic excitations and the other considers the impact of such excitations on jet characteristics. Most cases reviewed here pertain to cryogenic liquid jets with the environment, into which the jets are injected, existing at thermodynamic supercritical temperatures. This is similar to those experienced in cryogenic liquid rocket engines.

\section{Single Jet without External Excitation}

In this section, relevant experimental work conducted on a single steady round jet injected into an environment lacking any externally imposed acoustic disturbances is considered. The purpose here is to examine the behavior of such jets under high pressures, specifically supercritical conditions. In the selection of candidate cases, the test matrix is chosen to cover supercritical conditions. However, it is preferred that the matrix spans a broader range encompassing both suband supercritical conditions because it generally provides a more comprehensive picture of differences and similarities between the two conditions.

Historically, research on supercritical injection of relevance to liquid rockets started with a published work of Newman and Brzustowski [4]. They used a steady $\mathrm{CO}_{2}$ jet injected into a chamber of pure $\mathrm{N}_{2}$ and also into mixtures of $\mathrm{CO}_{2}+\mathrm{N}_{2}$ at both sub- and supercritical pressures and temperatures. Obviously, if the chamber is at a supercritical pressure, the injected jet pressure is higher and must also be at a supercritical pressure. They showed that when the chamber pressure approached just above the critical pressure of the $\mathrm{CO}_{2}$, injection of $\mathrm{CO}_{2}$ into mixtures of $\mathrm{CO}_{2}+$ $\mathrm{N}_{2}$ (varying initial $\mathrm{CO}_{2}$ concentration to change mixture density) widened the visual appearance of the jet. This was explained to be due to changes in chamber-to-injectant density ratio. At a higher chamber supercritical pressure, injection of $\mathrm{CO}_{2}$ into a pure nitrogen gas, but varying temperature (from sub- to supercritical), caused shortening of both the jet visible length and width with chamber temperature.

Newman and Brzustowski [4] also investigated and explained effects of increased chamber temperature on jet appearance. They found that such effects were due to progressive reduction in ambient gas density, hence lowering surface tension to zero at critical temperature, and to increase in liquid $\mathrm{CO}_{2}$ evaporation. In other experiments, $\mathrm{CO}_{2}$ was injected into a mixture of $\mathrm{CO}_{2}+\mathrm{N}_{2}$ with fixed but large initial $\mathrm{CO}_{2}$ mass fraction in order to reduce jet evaporation. The chamber temperature was fixed at a supercritical value, but its pressure was varied from sub- to supercritical pressures. They hypothesized and conjectured that at supercritical chamber temperatures and pressures the jet may be considered as a variable-density single-phase turbulent submerged gas jet. Finally, assuming self-preserving flow, negligible gravity, zero latent heat of vaporization, ideal gas behavior, and thermal equilibrium between gas and drops, they develop a model for predicting the profile of the outer extent of a supercritical steady jet and its centerline mean axial velocity. Comparison of this model with experiment was very poor near the injector exit area where most important and complex processes take place. Hence, the proposed hypothesis was not backed by this effort and the matter remained unresolved.

After the aforementioned initial study, two organizations (DLR in Germany and AFRL in USA) dominated the field by pursuing systematic research programs to understand jet breakup and dynamics under high chamber pressures at, and specifically exceeding, the critical condition of the injectant. The majority of the experimental works presented here is thus coming from these two organizations plus other satellite universities they collaborated and/or supported. Each organization has constructed a unique facility from which most of the results for nonreacting jets were acquired and presented here. For more details on their facility designs readers are referred to their publications, many of which are listed in the references.

Researchers at DLR began working with the most simplest and fundamental of all cases, that is, the injection of a single nonreacting round jet into a quiescent environment. At AFRL, although initially droplet studies were planned and conducted, a transition to jets at high Reynolds numbers of practical interest was initiated by the author of this paper and his team members; for example, see Chehroudi et al. [5]. The injection of jets were studied at various chamber pressures ranging from subcritical to supercritical (mostly at supercritical temperatures), which included chamber pressures representative of those experienced in typical cryogenic liquid rocket engine (LRE). For safety reasons, and for the $\mathrm{H}_{2} / \mathrm{LOX}$ liquid rockets, it was preferred to simulate liquid oxygen with liquid nitrogen. Early studies have shown that at a nonreacting condition the injection behaviors of these two fluids were similar. To avoid complications introduced by mixture effects, however, many of these studies involved injection of cryogenic liquid nitrogen $\left(\mathrm{LN}_{2}\right)$ into room temperature gaseous nitrogen $\left(\mathrm{GN}_{2}\right)$. Critical pressure and temperature of nitrogen are $3.39 \mathrm{MPa}$ and $126.2 \mathrm{~K}$, 
respectively. Injections into other ambient gases were also investigated.

The objective of this section of the paper is to present key findings and discuss representative results. In some cases, key conclusions confirmed independently by both organizations (DLR and AFRL) are highlighted when appropriate and if they add to the reader's understanding. Before delving into details of the results, it worth indicating that Mayer et al. [6] were the first to take active steps towards a largescale facility investigating both single and coaxial cryogenic jets under cold and fired conditions. They used $\mathrm{LN}_{2}$ jets at $105 \mathrm{~K}$ injected into a $\mathrm{GN}_{2}$ environment at $300 \mathrm{~K}$, but at varying ambient pressures ranging from sub- to supercritical conditions. They reported drastic changes in the jet structure near and above the critical pressure. The jet behaved similar to the classical atomization of liquid fuels, with ligaments and drops, below the critical pressure. Mayer et al. [6] attributed this behavior to a continual decline of surface tension until it vanished at and beyond the critical point.

2.1. Visualization of the Jet Interface. Figure 1 presents results published in a work led by Chehroudi which shows representative images of cryogenic $\mathrm{LN}_{2}$ jets injected into gaseous nitrogen at $300 \mathrm{~K}$ (supercritical in temperature); see Chehroudi et al. [5]. The initial temperature of the jets was measured in a separate experiment and under identical flow conditions with a very small thermocouple. Depending on the flow condition, the measured initial injection temperature varied from $99 \mathrm{~K}$ to $110 \mathrm{~K}$, that is, injected at a subcritical value. Pressures in Figure 1 are reported as reduced pressures $\left(P_{r}\right)$, defined to be the chamber pressure divided by the critical pressure of the injected nitrogen. In frames 1 to 4 (of Figure 1), where the chamber pressure is subcritical, the jets have a classical liquid spray appearance. Figure 2 shows magnified images of the three injection cases under sub-, near-, and super-critical chamber pressures. This software magnification is performed to more clearly show the shear layer structure near the injector exit area. As shown in Figure 1, and consistent with the classical liquid jet breakup regimes described by Reitz and Bracco [7], surface instabilities grow downstream from the injector, and very fine ligaments and drops are ejected from the jet (see also the left image in Figure 2). This behavior corresponds to the second wind-induced liquid jet break-up regime described by Reitz and Bracco [7].

Major structural and interfacial changes occur at about $P_{r}=1.03$ as shown in frame 5 of Figure 1. Above this chamber pressure, drops are no longer detected, and as characterized by Chehroudi et al. [5], regular "finger-like" entities are observed at the interface. Rather than breaking up into droplets, the interface appears to dissolve at different distances from the dense and dark core. These structures are illustrated at $P_{r}=1.22$ in the middle frame of Figure 2. Such a change in morphology of the mixing layer is evidently due to combined effects of the reduction in the surface tension, as the critical pressure is exceeded, and disappearance of the enthalpy of vaporization because of this transition to supercritical pressures.
As the chamber pressure is further increased, the length and thickness of the dense (and dark) core decrease, and the jet begins to appear similar to a turbulent gaseous jet injected into a gaseous environment. This is illustrated in frames 7 and higher in Figure 1. Any further droplet production, and consequently any additional classical liquid atomization, is completely suppressed. These observations were confirmed by Mayer et al. [6, 8], Chehroudi et al. [5], and Roy and Segal [9]. As mentioned earlier, similar results were also found when injecting liquid oxygen instead of the liquid nitrogen.

It is important to indicate that because of the very large density variations between the jet core and the chamber, Chehroudi et al. [5] investigated whether the evolution of the jet within the region of their measurement was affected by the buoyancy forces. Therefore, they calculated the Froude number values under each test condition. As an example, Chehroudi et al. $[5,10]$ showed that the Froude number ranged from 42,000 to 110,000 . To make sense of these values, they looked at the Chen and Rodi [11] results. Chen and Rodi [11] suggested that the flow is momentum dominated when a defined length scale $x_{b}$ is less than 0.53 , while Papanicolaou and List [12] suggested $x_{b}<1$. The length scale is given by $x_{b}=\operatorname{Fr}^{-1 / 2}\left(\rho / \rho_{\infty}\right)^{-1 / 4}(x / d)$, where $x$ is the axial distance, $d$ is the initial jet diameter, and $\rho$ and $\rho_{\infty}$ are the jet and ambient densities, respectively. The Froude number is defined as $\mathrm{Fr} \equiv \rho U^{2} / g d\left|\rho_{\infty}-\rho\right|$ where $U$ is the velocity difference and $g$ is the gravitational acceleration. Considering a more conservative estimate by Chen and Rodi [11], the jet used by Chehroudi et al. $[5,10]$ is momentum dominated for distances less than 30 to $40 \mathrm{~mm}$ from the injector exit plane. Pictures presented in Figures 1 and 2 cover up to about $5.5 \mathrm{~mm}$ (axial distance/diameter ratio of 21.6) from the injector, and hence buoyancy effects can be ignored in favor of inertial forces.

2.2. Length Scale Investigation. Injection of a single $\mathrm{LN}_{2}$ jet into gaseous $\mathrm{N}_{2}$ (at $298 \mathrm{~K}$ ) has been investigated by Branam and Mayer [13] at ambient pressures of $4 \mathrm{MPa}$ and $6 \mathrm{MPa}$, corresponding to reduced pressures of 1.17 and 1.76 , respectively. The initial injection temperature of the nitrogen was near the critical point. They provided a measure of the length scales by analyzing shadowgraph images and called it as the "visible length scale." More details on their image analysis can be found in Branam and Mayer [13].

Figure 3 shows a typical result of the geometrically averaged length scale (average of radial and axial length scales) measured at the $x / D$ of 10 as a function of the radial position. Results from the $k-\varepsilon$ computational method are also shown. This suggests that the measured visible length scale is comparable in magnitude to the Taylor length scale determined by the computational method.

The ratio of the axial to the radial length scales indicates whether the visible structures are spherical or more ellipsoidal in shape. Both length scales are shown in Figure 4 for an injection temperature of $123 \mathrm{~K}$, that is, injection of $\mathrm{N}_{2}$ at a high density. In the near-injector region, the axial length scales are much larger than the radial ones. Further downstream, however, the visible structures become more 

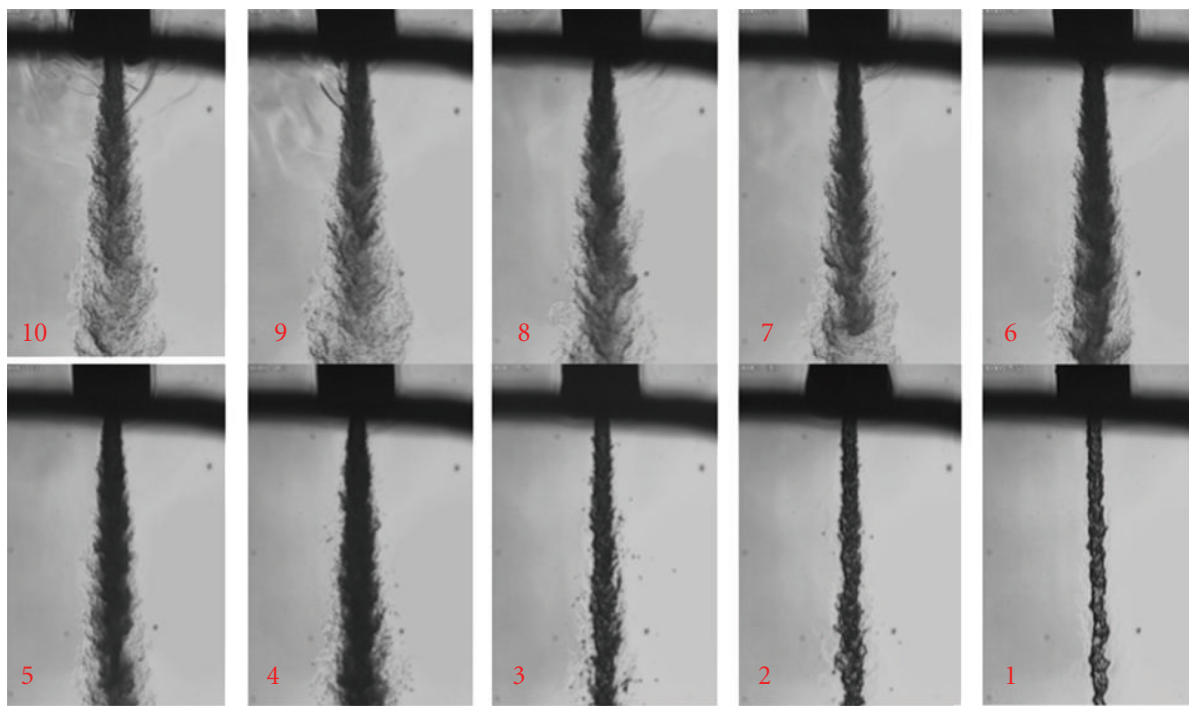

Figure 1: Back-illuminated images of a single nitrogen jet injected into nitrogen at a fixed supercritical temperature of $300 \mathrm{~K}$ but varying sub- to supercritical pressures (For $\mathrm{N}_{2}: P_{\text {critical }}=3.39 \mathrm{MPa} ; T_{c}=126.2 \mathrm{~K}$ ). From lower right to upper left: $P_{\text {ch }} / P_{\text {critical }}($ frame no.) $=0.23$ (1), 0.43 (2), 0.62 (3), 0.83 (4), 1.03 (5), 1.22 (6), 1.62 (7), 2.44 (8), 2.74 (9). Reynolds' number (Re) was from 25,000 to 75,000. Injection' velocity: $10-15 \mathrm{~m} / \mathrm{s}$. Froude' number: 40,000 to 110,000 . Injectant temperature: 99 to $120 \mathrm{~K}$. Chehroudi et al. [5].

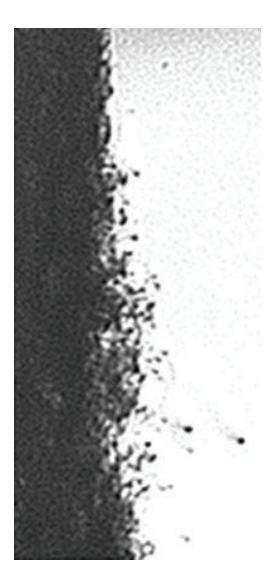

Appearance of conventional breakup of liquid surface indicating ligaments and drops ejecting from the mixing zone

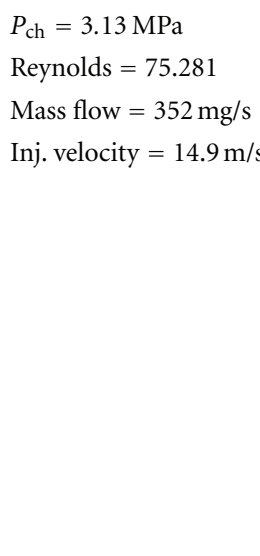

$P_{\text {ch }}=3.13 \mathrm{MPa}$

Reynolds $=75.28$

Mass flow $=352 \mathrm{mg} / \mathrm{s}$

Inj. velocity $=14.9 \mathrm{~m} / \mathrm{s}$

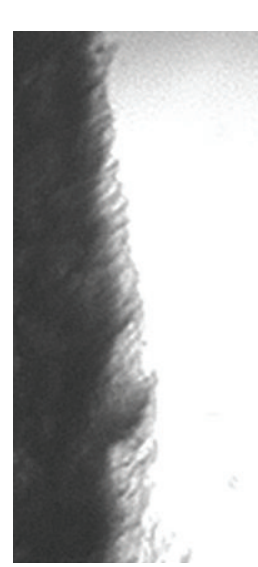

Mixing layer affected

by sub- to

supercritical

transition. No drops

are seen

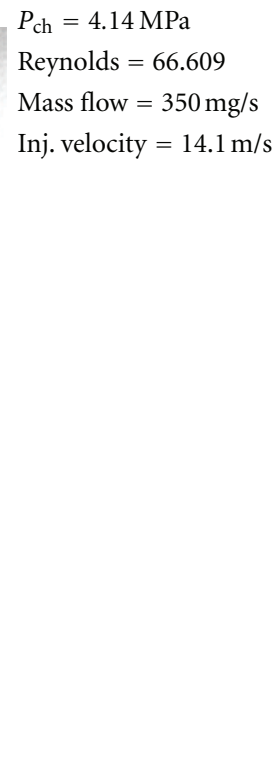

$P_{\mathrm{ch}}=4.14 \mathrm{MPa}$

Reynolds $=66.609$

Mass flow $=350 \mathrm{mg} / \mathrm{s}$

$P_{\mathrm{ch}}=9.19 \mathrm{MPa}$

Reynolds $=42.83$

Mass flow $=350 \mathrm{mg} / \mathrm{s}$

Inj. velocity $=11.7 \mathrm{~m} / \mathrm{s}$

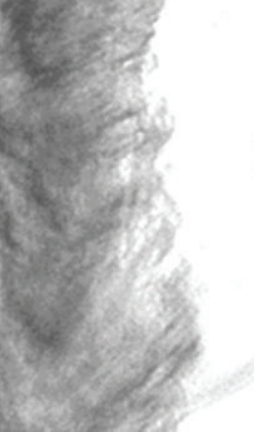

Appearance of

gas/gas mixing

layer

FIgURE 2: Software magnified images of the jets in Figure 1 at their outer boundaries showing transition to the gas-jet-like appearance starting at just below the critical pressure of the injectant. Images are at fixed supercritical chamber temperature of $300 \mathrm{~K}$. Chehroudi et al. $[5]$.

circular in shape. At a higher injection temperature $(132 \mathrm{~K})$, the asymmetry between the radial and axial length scales is not as pronounced as that seen under the lower temperature condition (123 K).

2.3. Jet Spreading Angle or Growth Rate. Measurements and estimations of the growth rate of a jet have been a subject of intense research for years because it provides a primary measure of mixing and development of the jet itself. Chehroudi's group was the first to extract quantitative measurements of this physical parameter using the images taken from a cryogenic $\mathrm{N}_{2}$ jet injected into $\mathrm{GN}_{2}$ under both subcritical and supercritical pressures; see Chehroudi et al. [5]. These measurements led to important conclusions regarding the character of the growth rate and the behavior of the jet near the injector and under such conditions, specifically at supercritical chamber pressures.

The spreading angle or growth rate was measured from a field of view within $5.5 \mathrm{~mm}$ of the injector exit plane (distance-to-diameter ratio of up to 21.6) and was inertially 


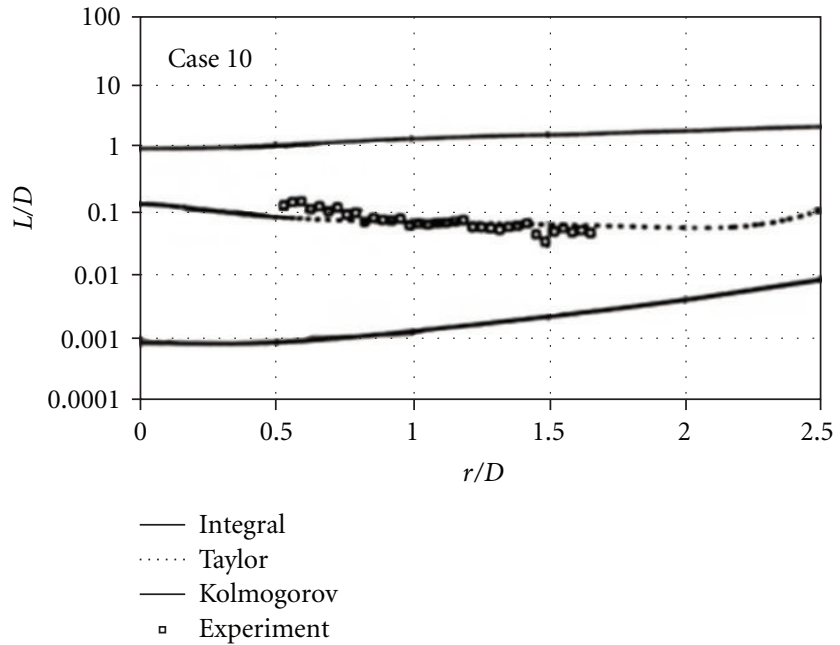

FIGURE 3: Comparison between calculated and experimental length scales for a single jet of $\mathrm{LN}_{2}$ injected into $\mathrm{GN}_{2}$ at $x / d=10$, chamber pressure of $6 \mathrm{MPa}, 1.9 \mathrm{~m} / \mathrm{s}$, injected temperature of $T=132 \mathrm{~K}$. Branam and Mayer [13].

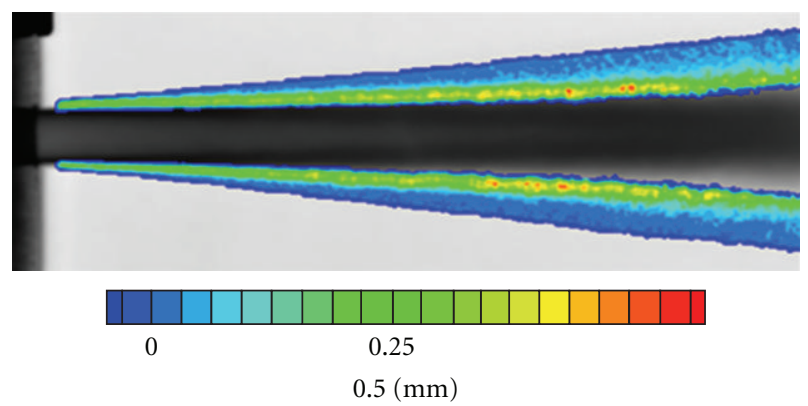

(a) Radial length scale

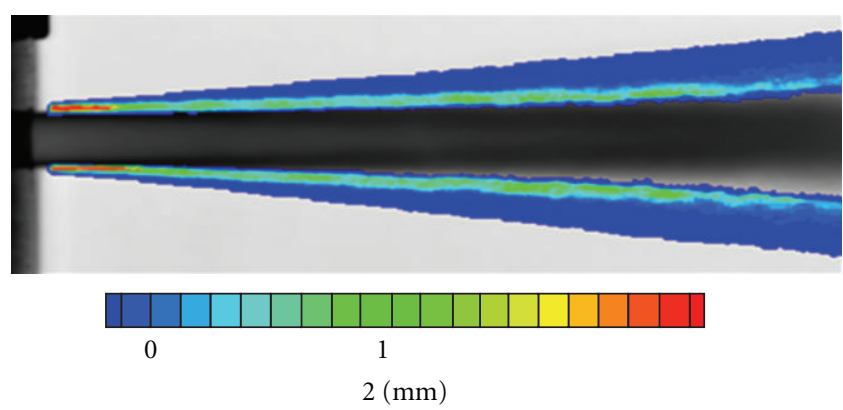

(b) Axial length scale

Figure 4: Experimental length scales for $\mathrm{LN}_{2}$ into $\mathrm{GN}_{2}$, chamber pressure of $4 \mathrm{MPa}, \mathrm{LN}_{2}$ injected temperature of $123 \mathrm{~K}$. Branam and Mayer [13].

dominated as discussed earlier. Chehroudi et al. [5] indicated that their data were also taken from the corresponding and appropriate initial region of the jet to ensure existence of a classical mixing layer. The initial jet spreading angle, or its growth rate, was then measured for all acquired images, and results along with those of others are presented in Figure 5. Of importance in this figure is the justification for the selection of the data sets and the nature of their

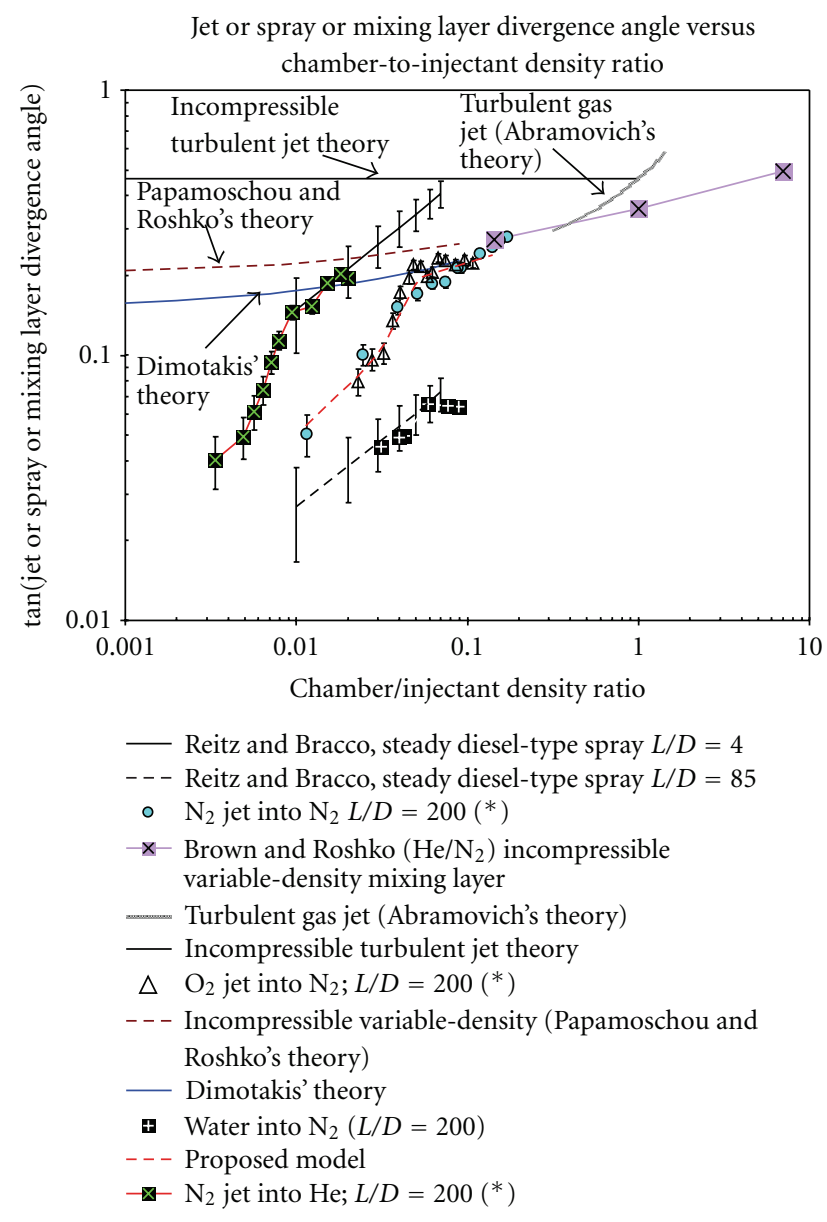

FIGURE 5: Spreading or growth rate of single jets as a tangent of the visual spreading angle versus the chamber-to-injectant density ratio. Data taken by Chehroudi are indicated by an asterisk $\left(^{*}\right)$ in the legend. Chehroudi et al. [5].

measurements by other researchers. They are elaborated at sufficient detail in earlier papers. In order to gain a deeper appreciation of these selections, the reader is referred to Chehroudi et al. [10]. Therefore, they are only mentioned here in brief.

Because the jets investigated by Chehroudi et al. [5] exhibited both liquid-spray-like and gas-jet-like (two-phase and single-phase, resp.) appearances, depending on pressure (see Figure 1), appropriate comparisons with both liquid sprays (injected into a gas) and gas jets (injected into a gas) were justified and hence these results are presented in Figure 5. The simplest is the prediction of the linear growth or constant spreading angle for the turbulent incompressible submerged jet using the mixing length concept. Following Abramovich [14], a semiempirical equation was used which attempts to incorporate the effects of density variations by an introduction of a characteristic velocity (see Chehroudi et al. [10] for more details). 
Brown and Roshko [15] measured spreading angles for a subsonic, two-dimensional, incompressible, turbulent mixing layer in which helium and nitrogen were used. Brown [16] (for a temporally growing mixing layer) and Papamoschou and Roshko [17] proposed a theoretical equation for incompressible variable-density gaseous mixing layers. Finally, Dimotakis [18] used the observation that, in general, the entrainment into the mixing layer from each stream was not the same and, in a system moving with a convection velocity, offered a geometrical argument to derive an equation for two-dimensional incompressible variable-density mixing layers. Chehroudi et al. [5] included predictions from these models as shown in Figure 5. Results by Richards and Pitts [19] for variable-density jets are also included.

Because both liquid-spray-like and gas-jet-like visual behaviors were observed, the growth rate for the liquid sprays produced from single-hole nozzles, typical of the ones used in diesel engines, was also incorporated in this figure. Figure 5 covers a density ratio of four orders of magnitude and is regarded as a unique and new plot in its own right. To some extent, and for comparable cases, disagreements between some results in this figure can be attributed to differences in the definition of the mixing layer thicknesses and the adopted measurement methods. For detailed discussion of this figure, see Chehroudi et al. $[5,10]$.

The important point which was stressed by Chehroudi et al. [10] is that for a range of density ratios in which images exhibit gas-jet-like appearance, the experimental data agrees well with the proposed theoretical equation by Dimotakis [18] and closely follows the trend of the Brown/Papamoschou and Roshko equation as shown in Figure 5. This can be taken as an important quantitative evidence that at supercritical pressures, the injected jets visually behave like a gas. Chehroudi's work appears to be the first time such a rigorous and quantitative evidence had been developed. The fractal dimension results discussed later provide additional evidence in support of this behavior.

Chehroudi et al. [20] also used the Raman scattering studies to measure density distributions. Initially, the growth rate measurements by them, using results acquired during the Raman scattering work, did not provide the same jet thickness values as those determined by the shadowgraphy approach. Apparently, as discussed by Brown and Roshko [15], different thickness definitions exist, and one can explore their relationship. Similar attempts in the context of supercritical jets showed that within the distances investigated, twice the full-width half-maximum (FWHM) of the Raman intensity radial profiles was equivalent to the thickness values measured through shadowgraphy. Realization of this relationship was very critical to consolidate the results from two different methodologies (Raman versus shadowgraphy). Figure 6 shows the growth rate measured using the Raman data in contrast to those determined through shadowgraph images.

These results were subsequently confirmed and extended by Oschwald and Micci [21] through a similar measurement technique. For example, they showed that when twice the FWHM of the Raman radial intensity profiles is used for

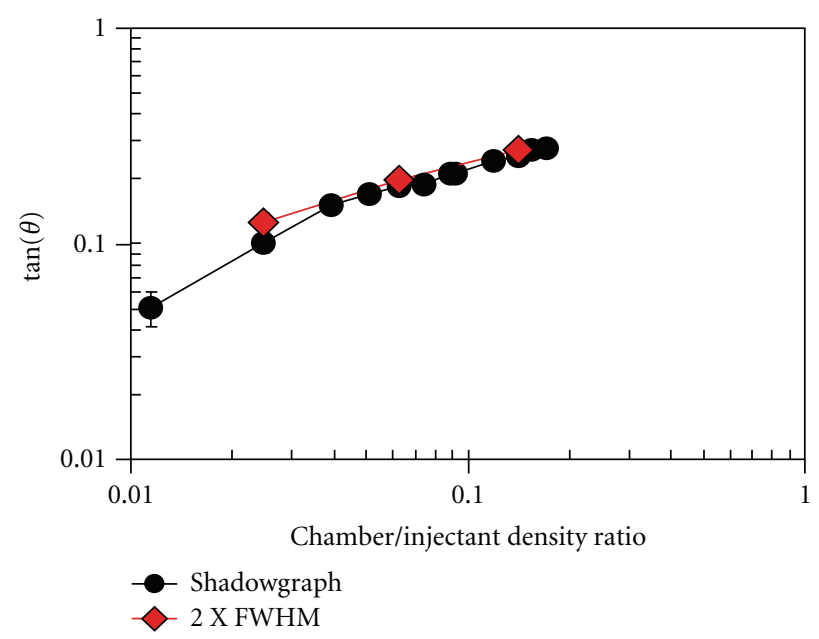

FIgURE 6: Comparison of the tangent of the spreading angle for a single jet of $\mathrm{LN}_{2}$ injected into $\mathrm{GN}_{2}$ measured using shadowgraph and Raman's techniques at twice the FWHM values. Chehroudi et al. [20].

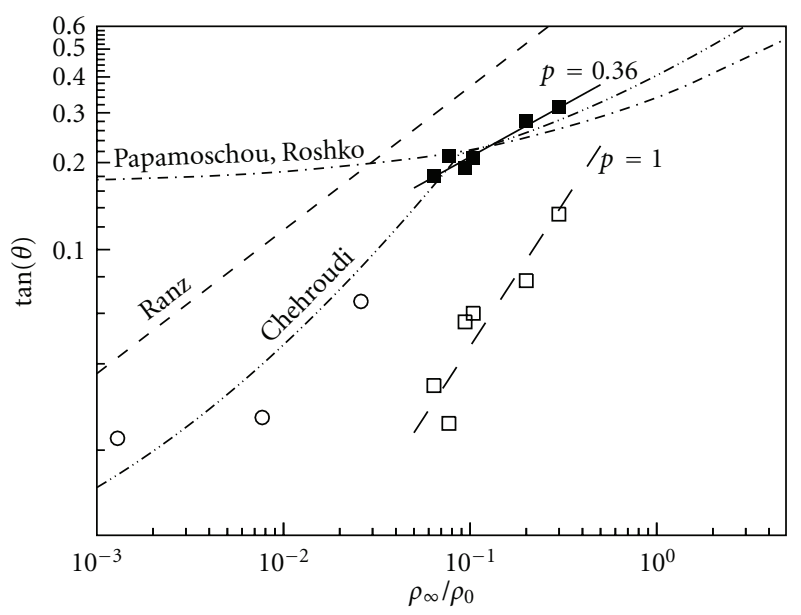

FIgure 7: Comparison of the tangent of the spreading angle by Raman's techniques using twice the FWHM values (DLR data). Solid squares are for data from $x / D$ of 15 to 32 whereas hollow squares are from $x / D$ of 0.5 to 14 . Chehroudi's model is also shown as dash-dot-dot curve. Single jet $\mathrm{LN}_{2}$ into $\mathrm{GN}_{2}$ with injector $L / D=$ 11.6. Data from Oschwald and Micci [21]. Open circles are data points from Reitz and Bracco [7].

$x / D$ values within 15 and 32, a good agreement exists between their results and those by Chehroudi's group (see the case designated as " $p=0.36$ " in Figure 7 , where " $p$ " is the exponent of the curve fitted through the data). However, the twice FWHM criterion did not fully agree with shadowgraphs for $x / D<15$ in the study conducted by Oschwald and Micci [21] (see the case identified as " $p=1.0$ " in Figure 7).

Note that Oschwald and Micci [21] measured a larger range of axial distances and found that the criterion was not universal at extended ranges. There are several reasons why different trends might be observed at different distances. 
It has to do with the fact that shadowgraphy and Raman scattering approaches measure different physical properties. The Raman signal is taken to be proportional to density whereas shadowgraphy is sensitive to the gradients of the density distribution. Thus a unique relationship between the results measured by the two methods may only be valid for a limited region and/or perhaps specific configurations. Recall that the data discussed above were obtained from injectors with different $L / D$ ratios. Another potential cause, and hence discrepancies, is errors in attempting to perform Raman's measurements close to the injector inlet where density variations and thus index of refraction variations can be very large.

2.4. Fractal Dimension of the Interface. Fractals are intimately connected to the concept of self-similarity; see Mandelbrot [22]. The fractal dimension of any curve is between 1 and 2 . The more wrinkled and space-filling a curve is, the larger the values of its fractal dimension. Natural curves, such as the outline of a cauliflower, are self-similar only to that within a narrow range of scales. The objective of the analysis here was to measure the fractal dimension of the interface of jets injected into the chamber in order to see if any pattern was uncovered.

The fractal dimension of jets at various pressures ranging from subcritical to supercritical was calculated and compared to results by other researchers. Reference results were taken from Sreenivasan and Meneveau [23] who measured the fractal dimensions of a variety of turbulent gaseous jets, mixing layers, and boundary layers. These results indicated a fractal dimension between 1.33 and 1.38. In addition, the fractal dimensions of a turbulent water jet (Dimotakis et al. [18]) and of a liquid jet in the second wind-induced atomization regime (Taylor and Hoyt [24]) were computed from high-resolution scanned images.

The fractal dimensions from the above reference cases are shown as horizontal lines in Figure 8. Overlaid on top of these lines are discrete points indicating the fractal dimension of $\mathrm{LN}_{2}$ jets injected into $\mathrm{GN}_{2}$ at various chamber pressures. One sees that at supercritical chamber pressures, the fractal dimension approaches a value similar to gaseous turbulent jets and mixing layers. As the chamber pressure is decreased, the fractal dimension also decreases. Below a reduced pressure of 0.8 , the fractal dimension rapidly declines to a value approximately equal to that of liquid sprays in the second wind-induced liquid jet break-up regime.

A detailed discussion of the above results is also found in Chehroudi et al. $[25,26]$. The key conclusion reached by Chehroudi's group is that the results from fractal analysis complement and extend the imaging data they acquired for the initial jet growth rate. At supercritical pressures, jets have a fractal dimension similar to turbulent gas jets, and at subcritical pressures, cryogenic jets have a fractal dimension similar to liquid sprays. The transition occurs at about the same chamber pressure as that when the transition in visual appearance and growth rate data discussed in Figure 5 takes place. Such distinctly different behaviors for jets under sub- and supercritical conditions were first demonstrated by

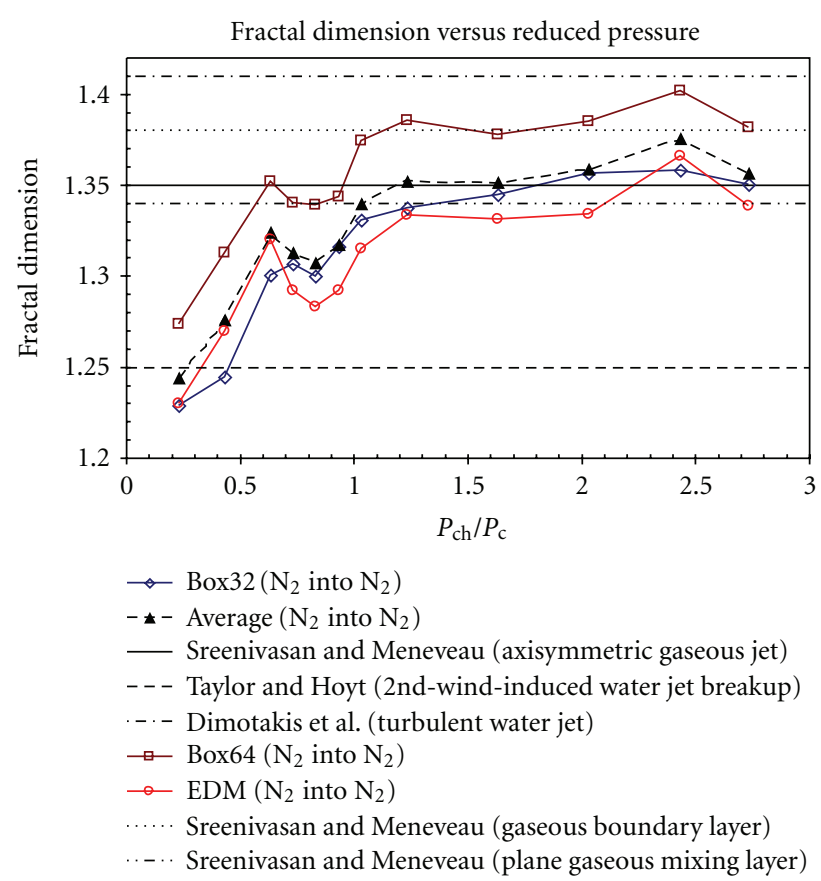

Figure 8: Fractal dimensions of the boundaries of various single jets as a function of reduced chamber pressure (chamber pressure divided by the critical pressure of the jet material). Discrete points are data from Chehroudi et al. [25, 26]. Box 32, Box 64, and EDM are different methods of calculating the fractal dimension, giving an impression of the extent of variability; for details, see Chehroudi et al. $[25,26]$.

Chehroudi's group in a quantitative manner using fractal analysis.

2.5. Measurement of the Dark-Core Length. Before presenting their results under supercritical conditions, Chehroudi et al. $[25,26]$ discussed some measurements from (gaseous and liquid) jets at subcritical conditions to set the stage and provide a contrast to their data. It is therefore useful to summarize what they recalled in their work.

According to Abramovich [14], the length of the "potential core" in isothermal uniform-density axisymmetric and two-dimensional jets is estimated to be about 6 to 10 injector diameters; whereas for nonisothermal cold jets injected into hot environments, it can reach up to about 25 injector diameters depending on jet temperature.

Also, according to Chehroudi et al. [27] the "intact core" length of the liquid sprays similar to the ones used in diesel engines is given by the equation $C d_{j}\left(\rho_{l} / \rho_{g}\right)^{1 / 2}$ where $\rho_{l}$ and $\rho_{g}$ are liquid injectant and chamber gas densities, respectively, $d_{j}$ is an effective jet exit diameter, and $C$ is a constant between 3.3 and 11. This reflects an intact core length between 33 and 110 injector diameters for the chamber-to-injectant density ratio of 0.01 and between 16.5 and 55 diameters for the chamber-to-injectant density ratio of 0.04 . These results are presented in Figures 9(a) and 9(b) for better comparison with what is measured for $\mathrm{LN}_{2}$ injection into both sub- and supercritical $\mathrm{GN}_{2}$ environment. 

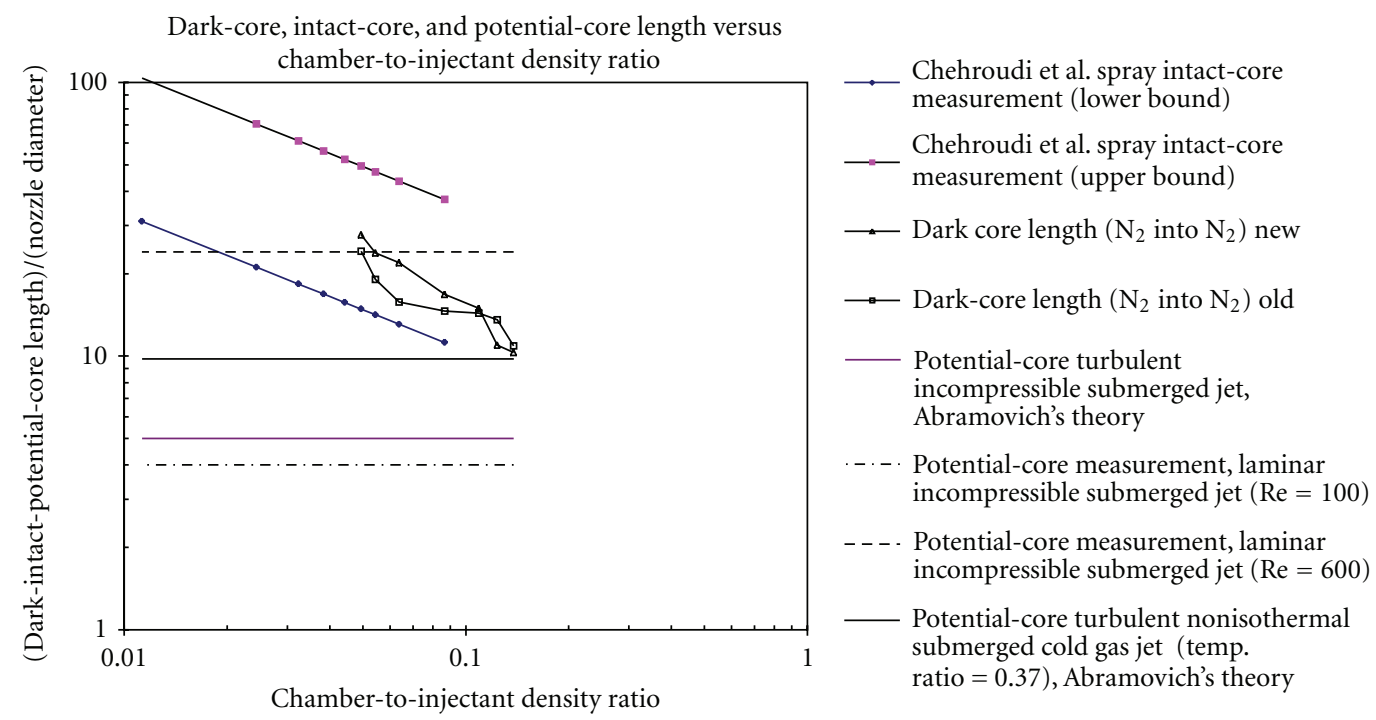

(a)

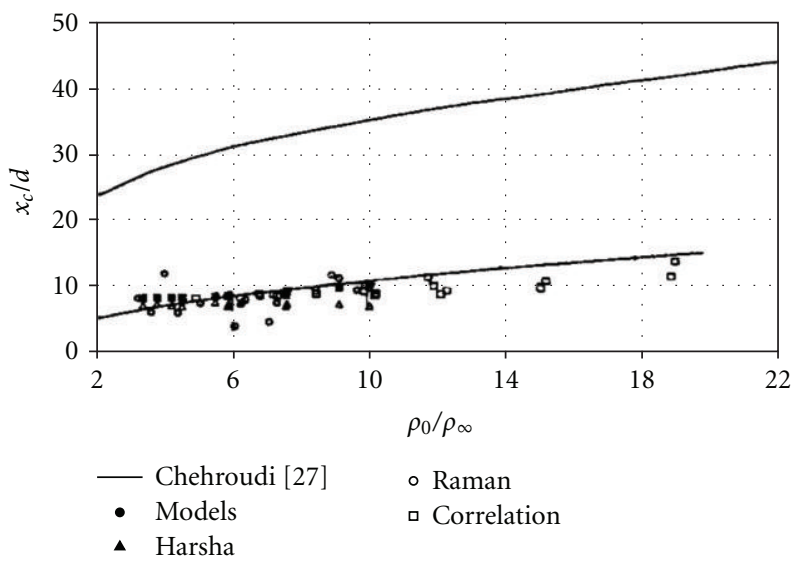

(b)

FIGURE 9: Ratio of either the dark-core, intact-core, or potential-core length, depending on the case, divided by the density ratio for single jets. (a): Chehroudi et al. [10], determined by analysis of shadowgraphs, for an injector $L / D=200$. (b): Branam and Mayer [13], determined via Raman data, injector $L / D=40$. Models: determined by computer simulation. Correlation: a correlation using a set of Raman's data by Branam and Mayer [13].

Considering that the classical two-stream mixing layer should start from the injector exit and extends to approximately the end of the potential core (or intact core) of the jet, Chehroudi et al. $[5,10]$ assumed that the jet "dark-core" region seen in their images played a similar role as the intact core or potential core. Figure 9(a) shows the dark-, intact-, or potential-core lengths normalized by the injector hole diameter plotted versus density ratio. By referring to Figure 9(a), Chehroudi et al. $[5,10]$ indicated that the growth rate data taken by them, which is presented earlier in Figure 1, was indeed from the corresponding and appropriate initial region to ensure existence of a classical mixing layer. They emphasized that it is only then when a valid comparison can be made (as they did) between their results and the two-stream mixing layers available in the literature. Finally, they found that the core length fluctuation levels at supercritical condition were several times lower than those observed at subcritical chamber pressures.

Complementary results by the Branam and Mayer [13] are also shown in Figure 9(b). These results were determined through a log-log plot of the centerline intensity measured by the Raman scattering to distinguish different flow regions. Note that the horizontal axis for Figures 9(a) and 9(b) are inverse of each other. The Branam and Mayer [13] data are at or below the lower bound of the Chehroudi et al. [27] model (i.e., solid curves in Figure 9(b)) which was proposed based on the liquid spray data in various atomization regimes. For chamber-to-injectant density ratios of less than 0.1 in Figure 9(a) (or injectant-to-chamber ratio of greater than 10 in Figure 9(b)), it appears that the Chehroudi et al. $[25,26]$ 
experimental data shown in Figure 9(a) is larger by about a factor of 1.5 to 2 compared to the "correlation" given by Branam and Mayer [13] in Figure 9(b).

Considering that the raw data used by the two groups are from two different injectors and measurement methodologies, the agreement is considered adequate. However, further investigations are warranted.

2.6. Density and Temperature Fields. The main purpose of the Raman scattering measurements was to provide quantitative information and to enable mapping of the jet density field. Temperatures, for example, were calculated assuming application of a suitable equation of state. Radial density profiles were reported by Oschwald and Schik [28] in a normalized fashion. In this section, the centerline density and/or temperature profiles as functions of the axial distance from the injector exit plane and their self-similarity assessment are discussed.

The test conditions were chosen in order to assess the influence of the thermodynamic state of the injected cryogenic $\mathrm{N}_{2}$ on the jet disintegration process. For example, above the critical pressure, the specific heat is finite but exhibits a maximum at a particular temperature. At this same point, the thermal diffusivity exhibits a minimum value. Three test cases were therefore investigated as shown in Figure 10(a). In test case A, the initial injection temperature is both above the critical temperature and above the temperature where the specific heat assumes a maximum value, whereas for test cases $B$ and $C$ the initial injection temperatures are both below the critical value and the temperature where the specific heat is at a maximum value.

Figures 10 (b) and 10 (c) show normalized centerline axial profiles of the density and temperature acquired by Oschwald and Schik [28] at a chamber pressure of $4 \mathrm{MPa}$ (near the critical pressure of nitrogen). Note that the density decay behavior becomes slower as the initial injection temperature is decreased. The temperature profile, however, stays flat for up to a normalized distance $(x / D)$ of about 25 to 30. They indicated that the development of the centerline temperature reflects the thermophysical properties of the nitrogen, being specific to the region where the specific heat reaches a maximum. For initial injection temperatures below the temperature where the specific heat reaches a maximum value, as the jet heats up, the fluid has to pass through a state with a maximum specific heat. The fluid temperature can then reach a value where a large amount of heat can be stored without any noticeable increase in temperature. It appears that the maximum specific heat line in a supercritical fluid results in a behavior similar to a liquid at its boiling point. That is, heat transfer to the nitrogen does not increase its temperature but simply expands the fluid (i.e., increases its specific volume). It is also for this reason that the dashed curves in Figure 10(a) are referred to as "pseudo boiling lines." Note that the density of the fluid varies strongly with temperature in this zone. At $6 \mathrm{MPa}\left(P_{r}=1.76\right.$, data not shown), the maximum of the specific heat is much less pronounced and the effects of the pseudo-boiling line is not as distinct as those seen under chamber pressure of $4 \mathrm{MPa}$. As shown by Oschwald and Schik [28], however, far downstream, it was observed that the temperature of the disintegrating and mixing supercritical fluid jet approached a value representative of a fully mixed jet but at a slower pace than that for the jet density.

The self-similarity of the density field has also been investigated by Chehroudi et al. [20] and the results are presented in Figure 11. According to Wygnanski and Fiedler [29], a fully self-preserved velocity field of a turbulent air jet should be observed at an $x / D$ of greater than 40 when the Reynolds number is near 100,000 . So et al. [30] reported selfpreservation for $x / D$ values larger than about 20 in a binary gas jet at Reynolds' number (Re) of about 4300 . Although it appears that some inconsistencies exist for this criterion, one can see that for the near-critical and supercritical pressures, the density radial profiles approach the similarity model curve shown in Figure 11. The disagreement increases at subcritical pressures where the model is least applicable. It is worth indicating that results from a modeling and computational simulation by Zong et al. [31] also agrees well with Chehroudi's Raman scattering measurements.

Chehroudi et al. [20] using their Raman scattering data determined the FWHM of the radial density profiles at each axial distance from the injector, and the results, along with data by other investigators, are shown in Figure 12. More information on their experimental conditions is given in Table 1. Note that except for Chehroudi et al. $[5,25]$ and Oschwald et al. [32], all others performed injection of gaseous fluids into an ambient gas at subcritical pressures (based on the injectant critical pressure). Also, the FWHM was determined using the mass fraction profiles in both So et al. [30] and Richards and Pitts [19]. However, reported FWHM values by So et al. [30] using both density and mass fraction profiles were comparable. Chehroudi et al. [20] data in Figure 12 shows an increasingly larger spreading rate as chamber pressure is raised. The data at the supercritical condition $\left(P_{r}=2.03\right)$ approaches that of Richards and Pitts [19] acquired at a density ratio of 1.56 even though this ratio is substantially smaller than Chehroudi et al. [20] data. Results at larger distances were not available for their jet to enable a more comprehensive comparison between the cases.

Richards and Pitts [19] concluded that if care is exercised to ensure that the flow is free of buoyancy and coflow effects, the spreading rate in variable-density jets was independent of the initial density ratio, velocity profile, and turbulence level and conformed with the constant-density results of others. In addition, they proposed a slope in the range between 0.212 and 0.220 for the linear jet growth rate equation; see Figure 12. However, a linear least-square fit to Chehroudi et al. [20] data at $P_{r}=2.03$ gives a slope of 0.102 , almost half of that by Richards and Pitts [19]. One possible explanation for this difference is that Chehroudi's data covers a range much closer to the injector exit plane than that by Richards and Pitts [19], leading to a lowered growth rate value. A tendency towards higher growth rates can be seen if only the farthest two data points are considered in Chehroudi's data. However, a solid conclusion cannot be drawn based on these two points. It is also possible that at some high enough injectant-to-chamber density ratio, the spread rate universality indicated by Richards and Pitts breaks down and 


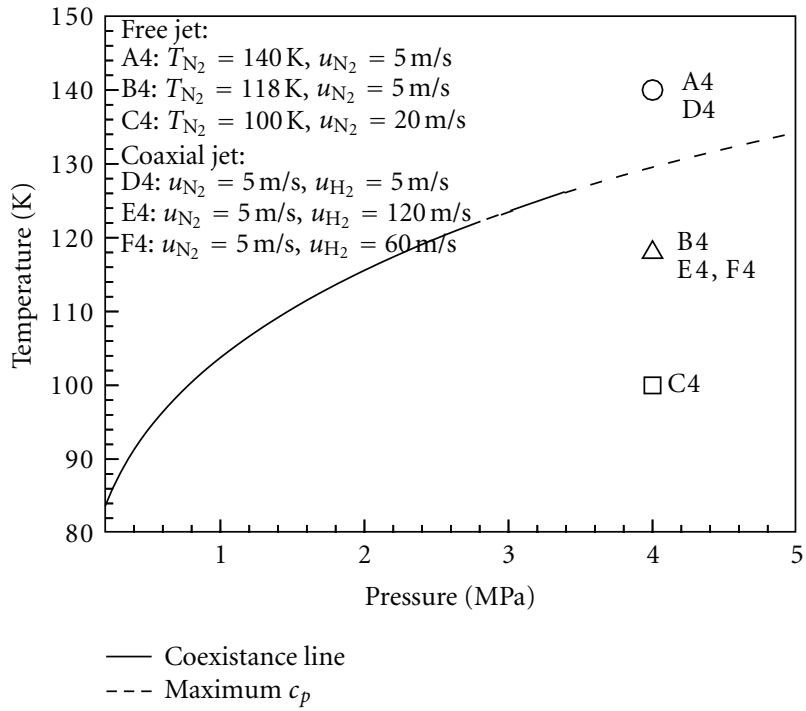

(a)

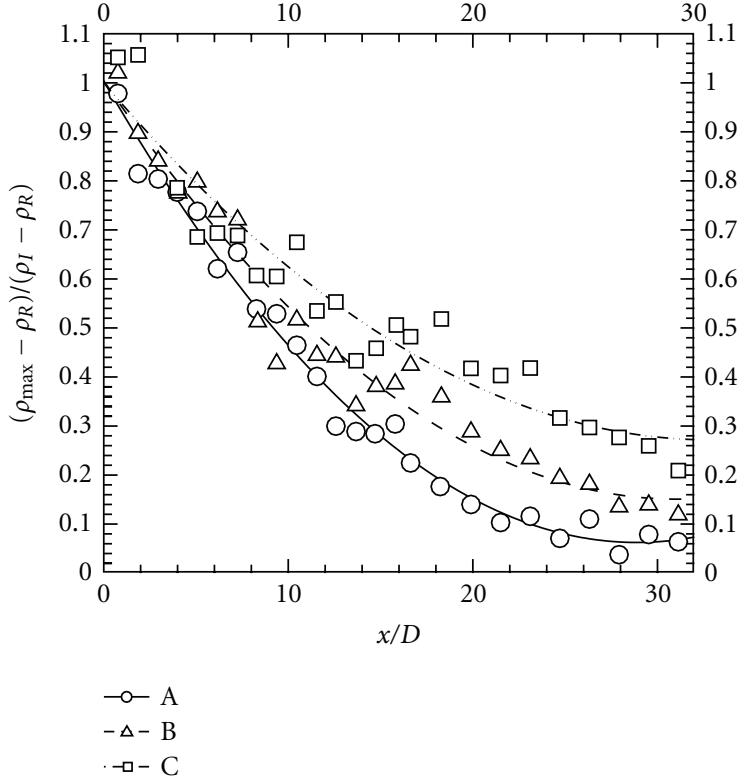

(b)

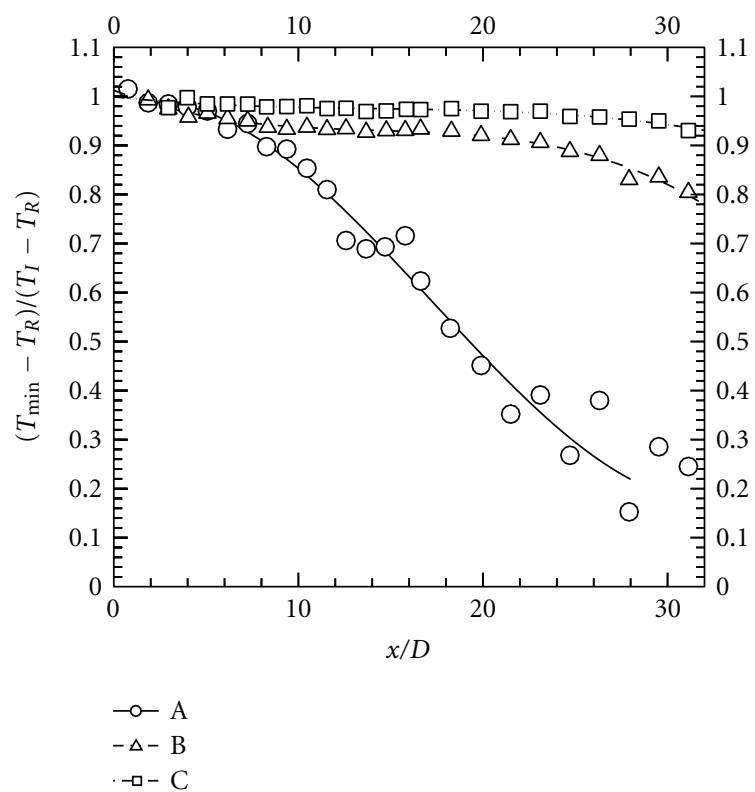

(c)

FIGURE 10: Normalized centerline density (b) and temperature (c) axial profiles of $\mathrm{LN}_{2}$ injected into $\mathrm{GN}_{2}$ at three different injection temperatures and a chamber pressure of $4 \mathrm{MPa}$ (i.e., near critical pressure). The plot (a) shows the thermodynamic conditions under which test cases A, B, and C are conducted. The dashed line is the pseudoboiling line. Note, A4, B4, and C4 symbols represent A, B, and C cases, respectively. Oschwald and Schik [28].

one observes a somewhat retarded growth rate for variabledensity turbulent jets. Some evidence in support of this position was given by Chehroudi et al. [20].

2.7. Phenomenological Model of the Jet Growth Rate. Using the experimental data collected on the growth of a cryogenic jet, a phenomenological model for the growth rate was proposed by Chehroudi et al. $[5,10]$ for the first time in the literature. Complete details on the development of this equation are to be found in these references. However, the physical reasoning motivating the proposed model equation is outlined below.

It was noticed by Chehroudi that previous expressions for the growth rate of liquid sprays and of turbulent jets have 
TABLE 1: Some information extracted from works by other investigators reported here. $T_{\mathrm{inj}}$ and $T_{\mathrm{ch}}$ are injection and chamber temperatures. $P_{\text {ch }}$ is chamber pressure. $L / D$ is the injector hole-to-diameter ratio, $x / D$ is the normalized distance from injector exit within which measurements were made. Chehroudi et al. [20].

\begin{tabular}{|c|c|c|c|c|c|c|c|c|c|c|c|}
\hline & (Fluid inj./cham.) & $\begin{array}{c}T_{\mathrm{inj}} \\
\mathrm{K}\end{array}$ & $\begin{array}{l}P_{\mathrm{ch}} \\
\mathrm{MPa}\end{array}$ & $\begin{array}{c}T_{\mathrm{ch}} \\
\mathrm{K}\end{array}$ & $\begin{array}{c}\text { Reduced } \\
\text { pressure } \\
P_{r}\end{array}$ & $\begin{array}{l}\text { Inj./chamb. } \\
\text { density ratio }\end{array}$ & $\begin{array}{c}\text { Diam } \\
D \\
\mathrm{~mm}\end{array}$ & $\begin{array}{l}\text { eter } \\
L / D\end{array}$ & $x / D$ & $\begin{array}{c}\text { Raynolds' } \\
\text { number } \\
\text { Re }\end{array}$ & $\begin{array}{l}\text { Profile used } \\
\text { to measure } \\
\text { FWHM }\end{array}$ \\
\hline Oschwald et al. & $\mathrm{N}_{2} / \mathrm{N}_{2}$ & 118 & 4 & 298 & 1.17 & 3.34 & 1.9 & 11.5 & 8.42 & $1.2 E+05$ & Density \\
\hline Oschwald et al. & $\mathrm{N}_{2} / \mathrm{N}_{2}$ & 140 & 4 & 298 & 1.17 & 12.5 & 1.9 & 11.5 & 1.05 & $1.3 E+05$ & Density \\
\hline Chehroudi et al. & $\mathrm{N}_{2} / \mathrm{N}_{2}$ & 95 & 6.9 & 295 & 2.03 & 7.1 & 0.505 & 100 & 4.8 to 24.4 & $3.5 E+04$ & Density \\
\hline Chehroudi et al. & $\mathrm{N}_{2} / \mathrm{N}_{2}$ & 110 & 1.5 & 295 & 0.43 & 40.6 & 0.505 & 100 & 4.8 to 24.5 & $1.2 E+04$ & Density \\
\hline So et al. & $(\mathrm{He}+\mathrm{Air}) /$ Air & 275 & 0.1 & 275 & 0.08 & 0.64 & 9.5 & & 5.1 & $5.0 E+03$ & $\begin{array}{l}\text { Concentration } \\
\text { and density }\end{array}$ \\
\hline So et al. & $(\mathrm{He}+\mathrm{Air}) / \mathrm{Air}$ & 275 & 0.1 & 275 & 0.08 & 0.64 & 9.5 & & 6.4 & $5.0 E+03$ & $\begin{array}{l}\text { Concentration } \\
\text { and density }\end{array}$ \\
\hline Richards and Pitts & He into Air & 275 & 0.1 & 275 & 0.44 & 0.138 & 6.35 & $\sim 50$ & $20-80$ & $4.0 E+03$ & Mass fraction \\
\hline Richards and Pitts & $\mathrm{C}_{3} \mathrm{H}_{8}$ into Air & 275 & 0.1 & 275 & 0.02 & 1.56 & 6.35 & $\sim 50$ & $40-120$ & $2.5 E+04$ & Mass fraction \\
\hline
\end{tabular}

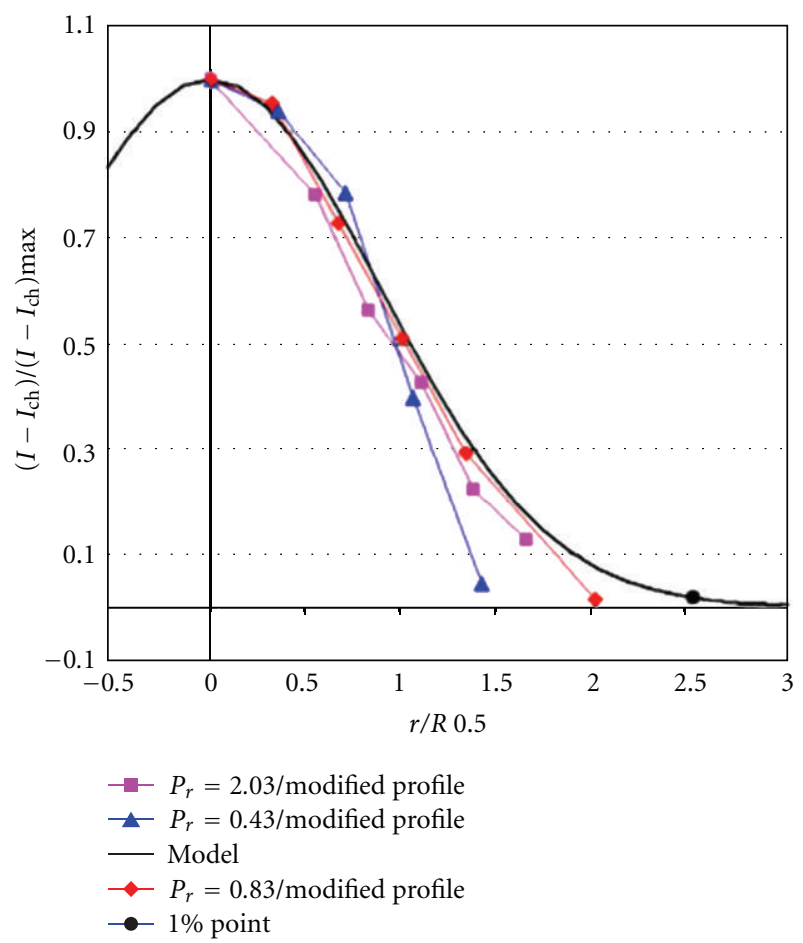

FIGURE 11: Plots of normalized intensity versus normalized radius for a single $\mathrm{LN}_{2}$ jet injected into $\mathrm{GN}_{2}$, at $x / D=12.2$, at subcritical, near-critical, and supercritical pressures (Chehroudi et al. [20]). The solid curve is the self-similar model that represents data from a gaseous jet injected into a gaseous environment. $I$ and $I_{\text {ch }}$ symbols are measured intensities in the jet and in the chamber far away from the jet, respectively. Chehroudi et al. [20].

a remarkably similar form. For example, Reitz and Bracco [7] proposed that the growth rate of isothermal steady liquid sprays could be expressed as

$$
\theta \approx 0.27\left[0+\left(\frac{\rho_{g}}{\rho_{l}}\right)^{0.5}\right] .
$$

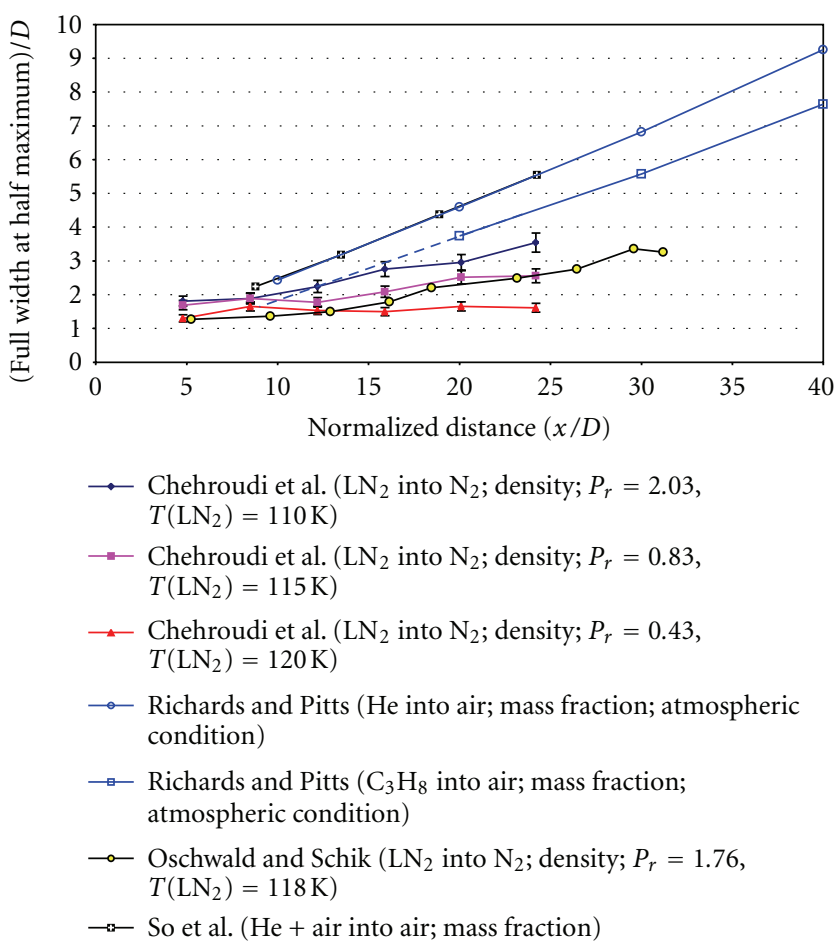

FIGURE 12: Normalized FWHM of the density-surplus radial profiles of single jets plotted as a function of the normalized axial distance from the injector exit plane. From Chehroudi et al. [20].

The first term in the bracket is the number zero. This zero term was purposely kept to enable a comparison with other equations Chehroudi et al. $[5,10]$ discussed. They also referred to an equation proposed by Papamoschou and Roshko [17] for incompressible, but variable-density, turbulent gaseous jets:

$$
\theta \approx 0.212\left[1+\left(\frac{\rho_{g}}{\rho_{l}}\right)^{0.5}\right] .
$$


The similarity in the form of these equations is quite astonishing considering vast differences between the cases. Chehroudi then suggested a linkage between the two cases. This is how Chehroudi et al. $[5,10]$ explained their case.

Imagine a jet that is being injected into a subcritical pressure environment similar to the ones shown in frame 4 of Figure 1 (or the left image in Figure 2). Clearly there are drops, ligaments, and interface "bulges" testifying existence of a surface tension. Chehroudi's group also reported evidence of a thermodynamic phase change under this condition. Hence, one appropriate characteristic time of the problem (at subcritical, $P_{r}<1$ ) is the "bulge" formation/separation time $\left(\tau_{b}\right)$ on the interface of the turbulent liquid jet. This time scale, according to Chehroudi et al. $[5,10]$, characterizes the formation and separation event of bulges from the liquid jet which subsequently produce isolated ligaments and drops. Then they referred to a suggestion made by Tseng et al. [33] that this time scale was equal to $\left(\rho_{l} L^{3} / \sigma\right)^{1 / 2}$ for the primary breakup in turbulent liquid jets, where $\rho_{l}, L$, and $\sigma$ are liquid density, characteristic dimensions of turbulent eddies, and surface tension, respectively. The second relevant characteristic time (for subcritical jets) that Chehroudi considered was the gasification time $\left(\tau_{g}\right)$. Here, an estimate of this time was calculated through the so-called $D$-squared law of spherical drop evaporation which is equal to $D^{2} / K$, where $D$ and $K$ are drop diameter and vaporization constant, respectively. In addition, Chehroudi also proposed a hypothesis as follows: if the aforementioned characteristic times (calculated for appropriate length scales) were nearly equal in magnitude, then the interface bulges are not able to separate as unattached entities from the jet interface to form ligaments and drops. This is because they are gasified as fast as they desire to be detached. This condition was then defined as the onset of the gas-jet-like behavior. Therefore, the transition between liquid-spray-like and gas-jet-like behaviors would be governed by finding the point at which these characteristic times are approximately equal. The scenario just explained is also supported by the "comb-like" structures seen in the middle image of Figure 2.

Using the above physical description, a model equation was then proposed for the $\mathrm{N}_{2} / \mathrm{N}_{2}$ system as

$$
\theta=0.27\left[\frac{\tau_{b}}{\left(\tau_{b}+\tau_{g}\right)}+\left(\frac{\rho_{g}}{\rho_{l}}\right)^{0.5}\right] .
$$

In the limit when $\tau_{g} \gg \tau_{b}$ and $\tau_{g} \rightarrow \infty$, this equation collapses to the isothermal liquid spray case. This equation agrees well with the Chehroudi et al. $[5,10]$ experimental data at subcritical pressures for $\tau_{b} /\left(\tau_{b}+\tau_{g}\right)<0.5$. A constant value of 0.5 was used for this term to predict the spreading rate at higher chamber pressures, including supercritical pressures.

For injection of $\mathrm{N}_{2}$ into $\mathrm{N}_{2}$, the characteristic time ratio, $\tau_{b} /\left(\tau_{b}+\tau_{g}\right)$, was computed from the experimental measurements of bulge and droplet sizes and calculations of the relevant properties. For $\mathrm{N}_{2}$ injection into other gases, however, reliable information about the mixture properties at the interface, particularly the surface tension, prevented

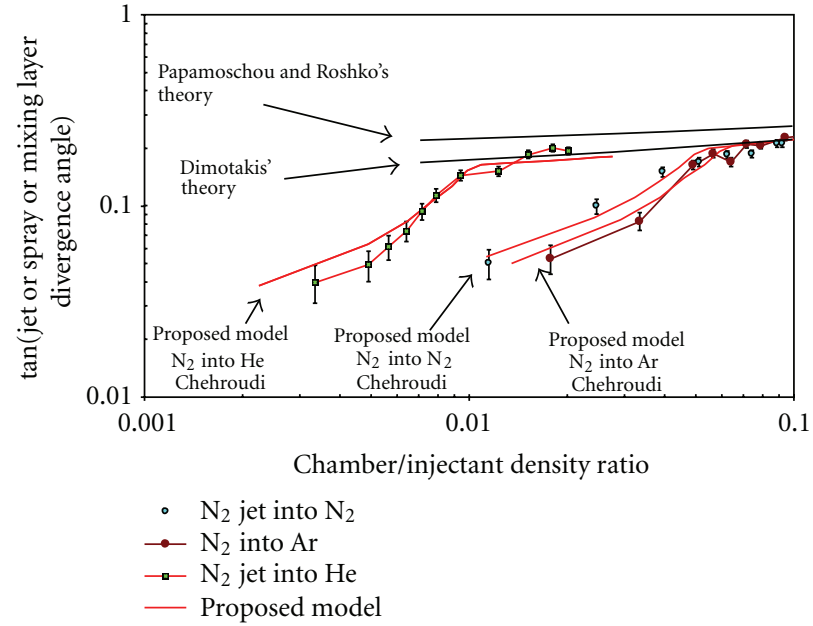

Figure 13: Comparison of the Chehroudi's proposed growth rate model with experimental data for single jets. Chehroudi et al. [5, $10]$.

such a calculation from being performed; see Chehroudi et al. $[5,10]$. To model these cases though, they further hypothesized that the characteristic time ratio term is a dominant function of the density ratio; that is, $\tau_{b} /\left(\tau_{b}+\tau_{g}\right)=$ $F\left(\rho_{g} / \rho_{l}\right)$. Chehroudi then referred to the work by Brown and Roshko [15] in which they indicated that such a hypothesis is reasonable. This is because at low Mach numbers, there is no distinction between mixing layers where the two streams have different molecular weights, temperatures, or compressibility effects. Measurements and calculations of $\tau_{b} /\left(\tau_{b}+\tau_{g}\right)$ provided a shape of the function " $F$ " for the $\mathrm{N}_{2} / \mathrm{N}_{2}$ system which is given as a plot in Chehroudi et al. $[5,10]$. A curve fit of that plot generates the following equation:

$$
\begin{aligned}
F\left(\frac{\rho_{g}}{\rho_{l}}\right) & =5.325\left(\frac{\rho_{g}}{\rho_{l}}\right)+0.0288 \quad \text { when } \frac{\rho_{g}}{\rho_{l}}<0.0885 \\
& =0.5 \quad \text { when } \frac{\rho_{g}}{\rho_{l}} \geq 0.0885 .
\end{aligned}
$$

It was found that the same function, $F$, calculated from measurements of the $\mathrm{N}_{2} / \mathrm{N}_{2}$ system could be made to work for other cases, provided that a case-dependent transformation was made to the density ratio at which the function $F$ is evaluated. The final form of the Chehroudi proposed equation is

$$
\theta=0.27\left[F\left(x\left(\frac{\rho_{g}}{\rho_{l}}\right)\right)+\left(\frac{\rho_{g}}{\rho_{l}}\right)^{0.5}\right],
$$

where $x=1.0$ for $\mathrm{N}_{2}$ into $\mathrm{N}_{2}, x=0.2$ for $\mathrm{N}_{2}$ into He, and $x=1.2$ for $\mathrm{N}_{2}$ into Ar.

The quality of the agreement with experimental data is demonstrated in Figure 13. Hence there are no major changes in the form of the proposed model equation, even for an extreme arrangement such as injection of $\mathrm{N}_{2}$ into He gas. It is also important to indicate that spreading angle 
under supercritical condition using modeling and computer simulation work by Zong and Yang [34] also agrees with Chehroudi's equation.

\section{Single Jet with External Excitation}

3.1. Effects of External Acoustic Field. Substantial evidences accumulated in the past attributed combustion instability to a complex interaction of the external acoustic field with the fuel injection processes, leading to incidences of instability in liquid rocket engines. For example, Oefelein and Yang [35] indicated that the near-injector processes in the thrust chamber were generally more sensitive to velocity fluctuations parallel to the injector face than normal to it. For this and other reasons, controlled experimental, analytical, and computational studies have been conducted in the past focusing on the effects of acoustic waves on both gaseous and liquid jets from a variety of injector designs. However, as indicated earlier, with a few exceptions, the scope of this paper is limited to experimental work which covers injection into supercritical conditions.

Chehroudi and Talley [36] used a piezo-siren design capable of generating sound waves with a sound power level (SPL) of up to $180 \mathrm{~dB}$. This was used at three chamber pressures of $1.46,2.48$, and $4.86 \mathrm{MPa}$ (reduced pressures of $0.43,0.73$, and 1.42 , resp.). The experimental setup consisted of an acoustic driver and a high-pressure chamber, forming a cavity which resonated at several frequencies. The strongest ones were reported at 2700 and $4800 \mathrm{~Hz}$. This basic setup was also used as the backbone for many of their future studies on coaxial injectors albeit with some improvements in data acquisition and facility plumbing. Chehroudi and Talley [36] injected $\mathrm{LN}_{2}$ into $\mathrm{GN}_{2}$ at room temperature under sub- and supercritical chamber pressures. Three different flow rates were considered, and the nature of the aforementioned interaction was documented via a highspeed imaging system using a CCD camera.

Figure 14 shows some sample results from this study where images of the jet are shown at two different perpendicular directions. These pair images at two different angles were taken on different test runs, but the operating conditions were as close as possible to each other. Each composite jet image consists of a mosaic of several images taken from the same test run but at different times and jet axial locations.

Chehroudi and Tally [36] reported that the acoustic field constricted the jet in the wave propagation direction, as seen in Figure 14, and consequently stretched the jet in the direction perpendicular to the propagation. They found that the impact of the acoustic waves on the jet structure was indeed strong at subcritical and near-critical pressures, but weakest at supercritical pressures. This is quite evident in Figure 14. They also observed that the externally imposed acoustic field interacted strongly at low injectant flow rates. The weakest effect was observed at supercritical pressures. This suggests that the mechanisms governing the coupling between acoustic waves and jets may be significantly different for jets injected under supercritical and subcritical conditions. This observation is important and its implications are discussed later in this writing.
To search for a plausible reason for the observed minimal impact at supercritical chamber pressures, Chehroudi and Talley [36] used the information mentioned earlier that at supercritical pressures, the unperturbed-jet spreading rate is the same as that by an incompressible variable-density gaseous jet; see Chehroudi et al. $[5,10]$. For this reason, one expects the existence of vortices similar to what has been described, for example, by Rockwell [37] in which transverse disturbances were generated by an externally oscillating plate at a fixed amplitude but different frequencies (1000 to $10000 \mathrm{~Hz}$ ). A synopsis of Rockwell's conclusions is useful in the context of the discussion here and hence is presented in the next two paragraphs.

A relatively large planar nozzle was designed by Rockwell [37] to inject water and investigate the details of vortex formation and their interactions near the exit area. The effects of the frequency of oscillations on the natural vortex coalescence were classified based on a Strouhal number (St $=f d / U$, where $f, d$, and $U$ are the externally imposed excitation frequency, jet diameter, and mean jet velocity) versus Reynolds number $(\operatorname{Re}=\rho U d / \mu$, where $\rho$ and $\mu$ are the injectant density and viscosity) plot, see Figure 15. For the range of Re investigated (1,860 to 10,800), natural breakdown of the jet (i.e., with no external disturbances) was observed to be within a few nozzle widths. The dimensionless frequency $\left(S t_{\mathrm{N}}\right)$ of natural vortices was found to change with Re number as $\mathrm{St}_{\mathrm{N}}=0.012(\mathrm{Re})^{0.5}$. Four regimes were distinguished based on the observed effects of the external disturbance on the nature of the vortex interaction. The reference regime was that which corresponds to the natural breakdown of the jet. The four regimes were named, in order of decreasing St number, as "upper zone," "preservation," "matched excitation," and "forced fusion" regimes. A plot of these regimes is shown in Figure 15 and compared with the values estimated for Chehroudi's study using $\mathrm{LN}_{2}$ injection into a $\mathrm{GN}_{2}$ chamber.

In the "upper zone" regime of Figure 15 where the excitation frequencies were higher than about 3 to 4 times the natural breakdown frequency $\left(\mathrm{St}>3 \mathrm{St}_{\mathrm{N}}\right.$ ), no effects were observed. In the "preservation" regime, the core flow of the jet tended to be preserved followed by the induction of smaller vortices, the time-averaged velocity profile was narrowed, and the longitudinal turbulence was decreased (relative to undisturbed case). When the excitation frequency was matched with the natural break-down frequency ("matched" regime), the effect was to accelerate the process of vortex formation and growth relative to the undisturbed case. Also, transverse distortion of the jet core was seen due to the vortex growth and coalescence. For the symmetrical jet work of Becker and Massaro [38], symmetrical ring vortices were produced and highest time-averaged widening of the jet has occurred. This was in contrast to the planar jet case in which vortices on the two sides of the jet were 180 degrees out of phase with each other. This regime merges to the "preservation" regime at higher Re number $(\sim 10,000)$. In the "forced fusion" regime (frequencies $\sim 1 / 3$ of natural jet breakdown), the natural break-down vortices were forced to fuse early as a result of the formation of largediameter applied disturbance vortices. Vaslov and Ginevskiy 

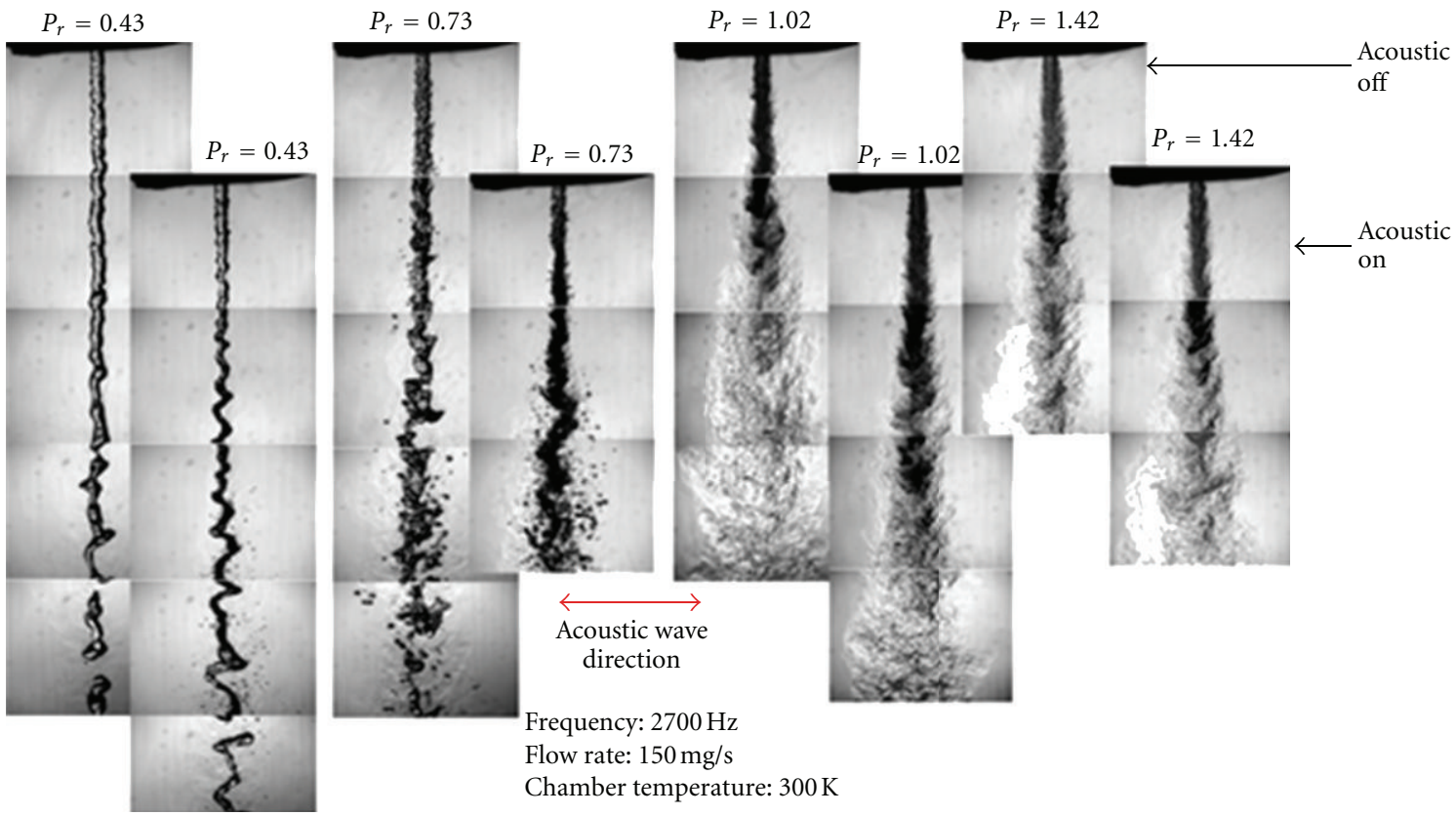

(a)

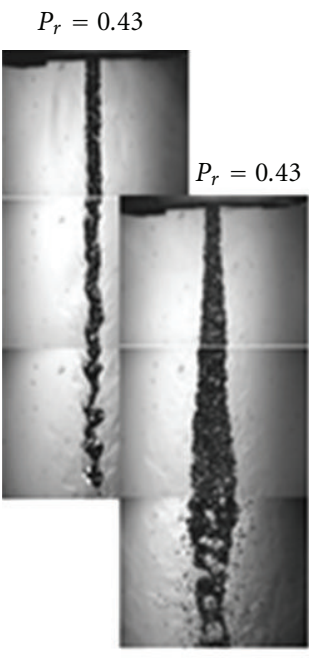

Frequency: $2700 \mathrm{~Hz}$ Flow rate: $150 \mathrm{mg} / \mathrm{s}$ Chamber temperature: $300 \mathrm{~K}$
$P_{r}=0.73$

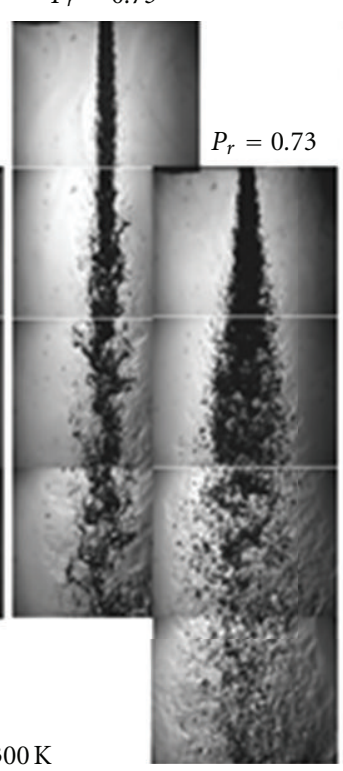

$P_{r}=1.02$

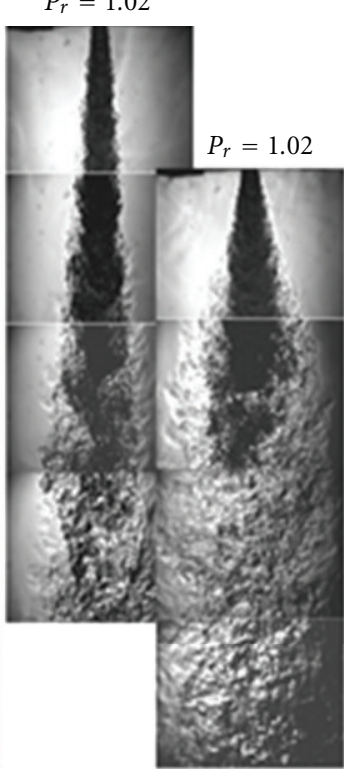

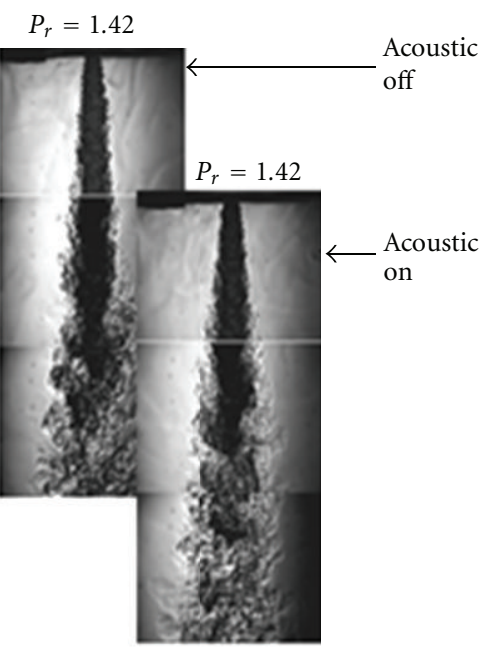

Acoustic wave normal to page

(b)

FIGURE 14: Interaction of acoustic waves with a single round liquid nitrogen jet injected into gaseous nitrogen at a range of chamber pressures from sub- to supercritical conditions. (a) Top images show front view; only one acoustic driver located at the left of the jet. The set of images shifted downward are when the acoustic field is turned on as indicated. (b) Bottom images show side views of the same jet (the same acoustic driver is located behind this page). $P_{r}$ is the reduced chamber pressure (i.e., chamber pressure divided by the critical temperature of the injected nitrogen). Chehroudi and Talley [36].

[39] found that in this regime the time-averaged velocity profile of the jet was broadened. Finally, in the "lower zone" regime (frequencies $<1 / 10$ of natural jet breakdown), the vortex growth was unaffected in their formation region. In the limit, however, as St approaches zero, the jet experienced a quasi-steady deflection process. Rockwell [37] stated that a fallacious averaged jet widening is detected if a time-averaged measurement technique is used downstream the injector, when in reality the jet is being deflected by the applied transverse disturbance.

To see how the results of Chehroudi and Talley [36] relate with Rockwell [37], their supercritical data is superimposed on the plot by Rockwell indicating different regimes discussed in the previous paragraph. Figure 15 shows a 


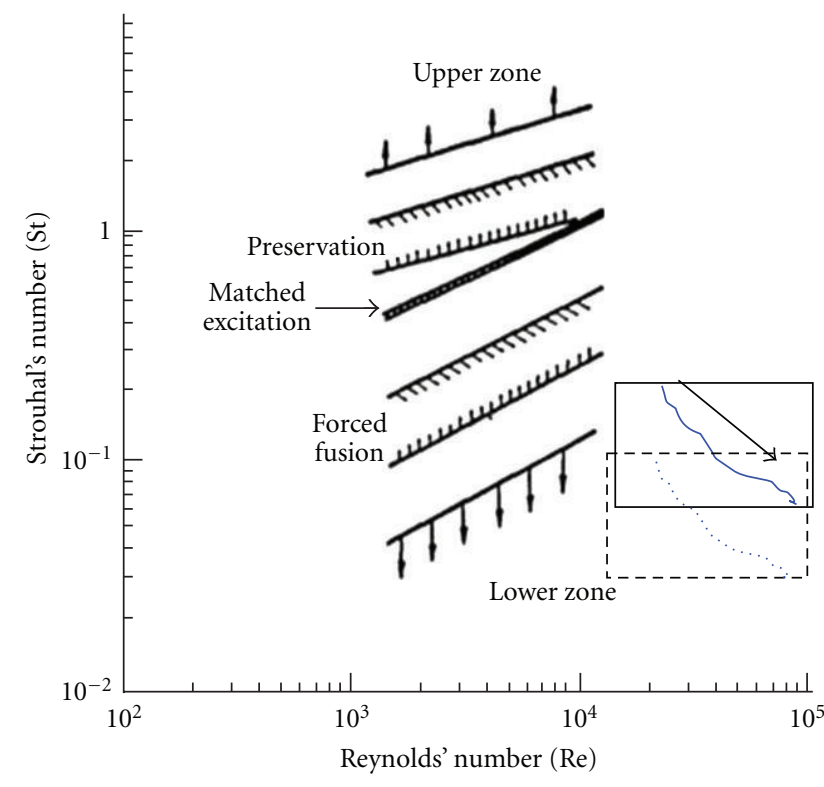

FIGURE 15: Regimes discussed by Rockwell [37] shown on an excitation Strouhal number versus the jet Reynolds number plot. The small solid rectangle to the right indicates the approximate region in which the Chehroudi $\mathrm{LN}_{2}$ jet data lies when excitation is at $4800 \mathrm{~Hz}$. The curve within this rectangle shows the approximate path as flow rate is increased from the lowest to the highest values, all at supercritical chamber pressures. Similar region for the $2700 \mathrm{~Hz}$ excitation is shown as a dashed rectangle. Chehroudi and Talley [36].

plot of the Strouhal number as a function of the Reynolds number. The curve marked as "matched excitation" is the same equation proposed by Becker and Massaro [38] for the natural breakdown of the jet. It can be seen that results from Chehroudi's study are beyond the regions studied by Rockwell [37]. With the caveats of extrapolating Rockwell's results, Figure 15 suggests that the frequencies used by Chehroudi's group are not expected to bring about noticeably large effects of the acoustic waves on supercritical jet because they lie in the region identified by Rockwell [37] as the "lower zone." No effects of the transverse disturbance on the jet were observed in the studies conducted by Rockwell [37] in this regime as described earlier. Note that the lowest flow rate case studied by Chehroudi's group in Figure 15 tends to enter into the "forced fusion" regime where stronger interaction is expected.

\section{Coaxial Jets without External Excitation}

Coaxial jets injectors have been used in liquid rocket engines since the 1940s and are found in the present-day hardware such as the Space Shuttle Main Engine (SSME); see Hulka and Hutt [40]. Rocket engines such as the SSME use liquid oxygen (LOX) and hydrogen $\left(\mathrm{H}_{2}\right)$ as propellants with the LOX flowing through an inner tube (or a center post) of a coaxial injector design and $\mathrm{H}_{2}$ through an outer annular region. The inner jet goes through the break-up process after it exits the injector and then mixes in the shear layer between the inner and outer streams. Subsequently, combustion and heat release occur to generate the desired engine thrust.

Often the combustion processes are controlled by, or at least intimately related to, the jet breakup and mixing of the oxidizer and fuel streams. Certain aspects of these processes can safely and inexpensively be studied using chemically inert fuel and oxidizer simulants. Moreover, attempts are made for as many parameters as possible, such as velocity ratio, mass flow rate ratio, density ratio, Reynolds' number $(\mathrm{Re})$, Weber's number (We), and Ohnesorge's number (Oh), to represent similar values obtained in real engines. This scaling exercise, to the extent possible, allows reasonable inferences and evaluation of different injector designs before ever being tested under hot fire conditions.

Investigations targeted at enhanced and fundamental understanding of the injection processes and combustion stability, although desirable to be performed under operating engine conditions, are practically problematic and costly for many reasons, including the need for an optically accessible full-scale liquid rocket engine, special material choices, and optical diagnostic limitations. For example, a singleelement noncombusting test rig having as much of the key features of a real cryogenic rocket engine as possible, such as cryogenic temperatures, transcritical temperature ranges, supercritical pressures, realistic velocity ratios, and activation of an acoustic field inside its test chamber, is as close as one can get to the actual engine, yet avoiding indicated complications associated with the combustion in a full-scale setup. Therefore, test rigs have been designed in many studies to have such desired characteristics. Combination of the cryogenic liquid $\mathrm{N}_{2}$ and gaseous $\mathrm{N}_{2}$ (to eliminate mixture critical phenomena) and $\mathrm{N}_{2}$ /helium (He) system (to better simulate the $\mathrm{LOX} / \mathrm{H}_{2}$ injection issues) were considered in most of these studies.

4.1. Coaxial $\mathrm{LN}_{2} / \mathrm{He}$ Injection. Telaar et al. [41] experimentally investigated the influence of the ambient pressure on the atomization phenomena from subcritical to supercritical pressures in a coaxial $\mathrm{LN}_{2} / \mathrm{He}$ injection. They used a coaxial injector design with an inner diameter of $d_{\mathrm{LN}_{2}}=1.9 \mathrm{~mm}$ for the cryogenic nitrogen flow and an annular slit width of $0.2 \mathrm{~mm}$ with an outer diameter of $2.8 \mathrm{~mm}$ for helium. Two examples of the flow visualization by shadowgraphy near the injector region are shown in Figure 16. The changes in the break-up mechanism at a reduced surface tension are quite evident. At the low chamber pressure of $1 \mathrm{MPa}$ in Figure 16(A), a liquid spray is formed, whereas at the supercritical pressure of $6 \mathrm{MPa}$ in Figure 16(B), a gas-like turbulent mixing of the dense and light fluids is seen. At $6 \mathrm{MPa}$, the initial temperature of the center $\mathrm{LN}_{2}$ jet was $97 \mathrm{~K}$, whereas the critical mixing temperature of $\mathrm{He} / \mathrm{N}_{2}$ mixtures at this pressure is $125.7 \mathrm{~K}$. Thus, in the mixing layer between the $\mathrm{LN}_{2}$ and $\mathrm{He}$ gas, transcritical zones may exist. The visual boundary of the $\mathrm{LN}_{2}$ jet as shown in this figure is assumed to be the layer at which the temperature reaches the critical mixing temperature. At this interface then, the influence of the surface tension forces as compared to the shear forces appears to be negligible. 


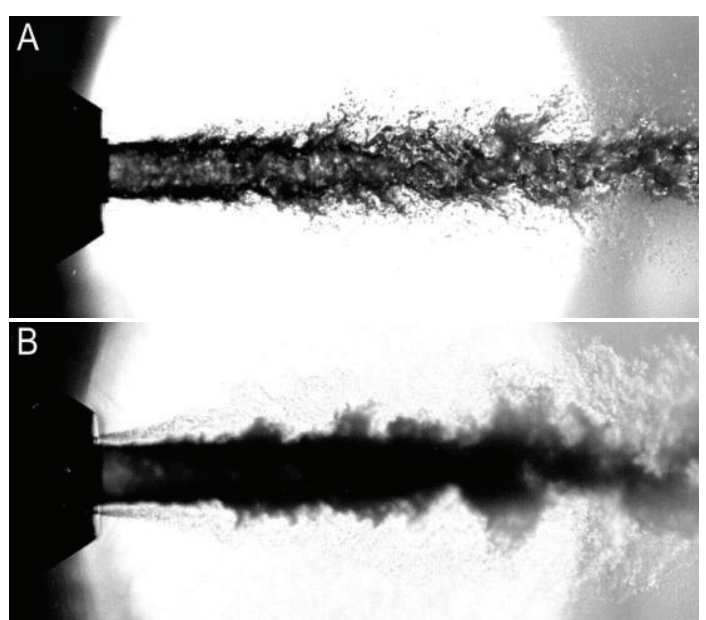

Figure 16: Binary coaxial liquid $\mathrm{N}_{2} / \mathrm{GHe}$ system at (A) chamber pressure of 1.0 MPa, (B) chamber pressure of 6.0 MPa. Inner-tube hole diameter, $d_{\mathrm{LN}_{2}}=1.9 \mathrm{~mm}$; liquid nitrogen injection velocity, $v_{\mathrm{LN}_{2}}=5 \mathrm{~m} / \mathrm{s}$; helium gas injection velocity, $v_{\mathrm{He}}=100 \mathrm{~m} / \mathrm{s}$; liquid nitrogen injection temperature, $T_{\mathrm{LN}_{2}}=97 \mathrm{~K}$; gaseous helium injection temperature, $T_{\mathrm{He}}=280 \mathrm{~K}$ (from Telaar et al. [41]).

4.2. Visualization of a Liquid Nitrogen/Gaseous Nitrogen Coaxial Injector. Davis and Chehroudi [42] used a coaxial injector designed to inject a liquid nitrogen, with a coflow of gaseous nitrogen in its annular region, as a part of a program to better understand the nature of the interaction between acoustic waves and liquid fuel jets in cryogenic rocket engines. In their test setup, injection was into a chamber filled with gaseous nitrogen at a supercritical temperature and different pressures spanning from sub- to super critical conditions. However, prior to presenting Davis and Chehroudi's results on acoustic forcing investigations, they provided a wealth of new information on dynamics of the jets (with no acoustic excitations), specifically focusing on the dark-core length of the inner jet in their coaxial injector. This is discussed next.

Davis and Chehroudi [42] argued that in their coaxial injector design, or in any generic one, the outer-jet flow rate played two key roles. First, it assisted the inner-jet breakup at the subcritical condition and enhanced mixing for the supercritical case. Second, because of the temperature differences between the inner jet and the outer jet, there was a heat exchange between the two, both inside and outside the injector. Evidences for the heat transfer behavior were given by examination of the results from jet temperature measurements. Figure 17 shows normalized temperature measurements within the inner jet and at the injector exit plane plotted versus normalized outer-jet mass flow rate at four different chamber pressures covering sub-, near-, and super-critical chamber pressures. Figure 18 shows images indicating typical jet characteristics taken at these same three-chamber reduced pressures. Evidences on the impact of the heat transfer are shown in Figure 17. For instance, at the lowest (subcritical) chamber pressure of $1.4 \mathrm{MPa}$, Davis and Chehroudi [42] found that the inner-jet exitplane temperature was fairly insensitive to changes in the

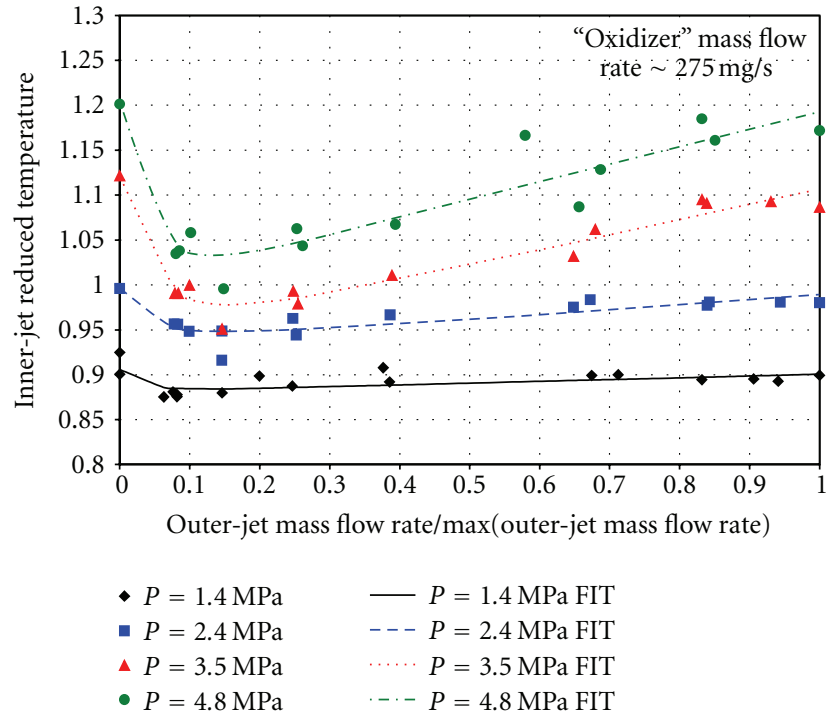

FIGURE 17: The inner-jet reduced temperature for a coaxial injector plotted versus the annular outer-jet mass flow rate, normalized by its maximum mass flow rate, at four different chamber pressures. Inner-jet mass flow rate is fixed at about $275 \mathrm{mg} / \mathrm{s}$. The lines, called "FIT" on the inset, are curve fits to the raw data. The maximum outer-jet mass flow rates for 1.4, 2.4, 3.5, and 4.8 MPa are 2,995, 2,985, 2,974, and 2,918 mg/s, respectively, Davis and Chehroudi [42].

annular outer-jet flow rate; see Figure 17. However, at all other elevated pressures, particularly at the supercritical one, an increase in inner-jet exit temperature was measured.

Under zero or no outer-jet flow rate condition in Figure 17, the annular passage of the injector was filled with the warm chamber nitrogen, hence strongly affected the heat transfer not only inside but also outside the injector. Clear evidence for the high heat transfer rate inside the injector was shown from the exit temperature measurements under this no-flow condition; see the $y$-axis in Figure 17. Here, the observed elevated temperature was reported to be the effects of the warm chamber gases present inside the annular space of the injector. Hence, referring to Figure 18, even though no atomizing (outer-jet) mass flow was at work in frames 11 and 21, one sees a shorter dark-core axial length than those seen in other images presented in frames 13 and 23. A small flow of the colder-than-chamber gaseous nitrogen in the annular passage, though may not assist the jet breakup, lowers the inner-jet exit temperature. Davis and Chehroudi [42] reported that the two processes (i.e., gas-assist liquid atomization and heat transmission) worked in such a way that the net effect was an apparent longer dark core in most cases. For example, compare the images in column one and two (from the left) in the last two rows as shown in Figure 18.

Clearly, the jet behaved differently at different outer-jet mass flow rates. Considering that the outer-jet mass flow rate acted as an atomizing gas, thus accelerating the breakup mechanism, then as the mass flow rate of the outer jet increased slightly $(\sim 487 \mathrm{mg} / \mathrm{s}$, frame 3 , Figure 18$)$, the inner jet began to break up into relatively large droplets and 


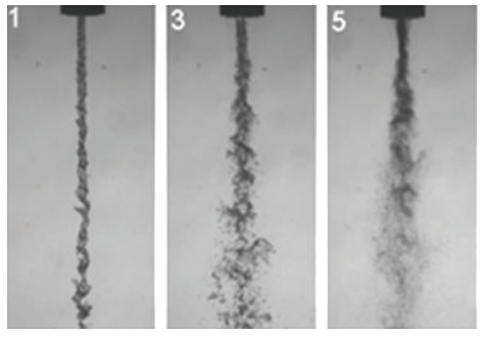

(a)

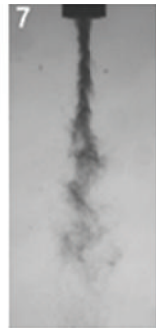

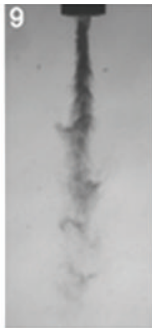
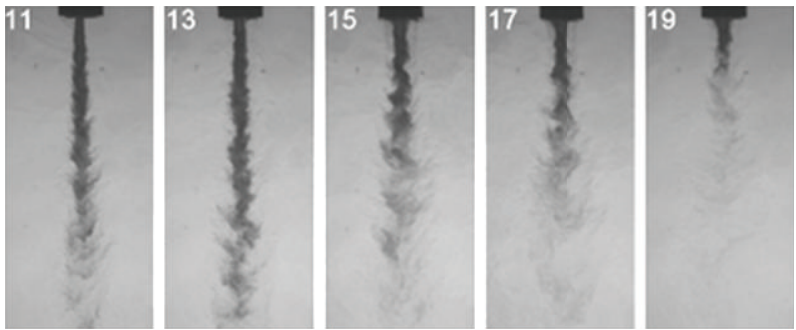

(b)
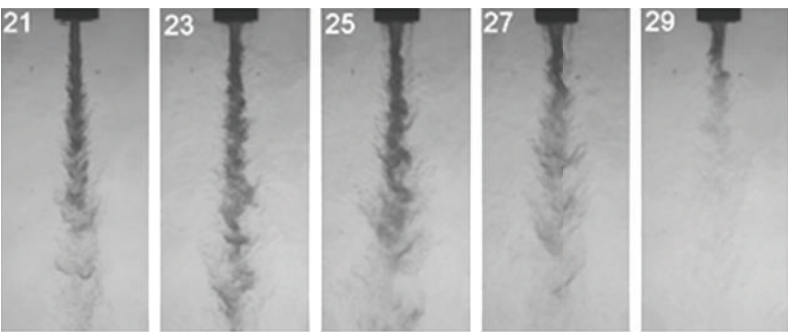

(c)

FIgURE 18: Images of a coaxial jet at approximately the same inner-jet mass flow rates $(\sim 275 \mathrm{mg} / \mathrm{s})$. Columns are at about the same outerannular-jet mass flow rates. For each row, the annular mass flow rate starts at a zero value to $2800 \mathrm{mg} / \mathrm{s}$ and increases from the left column to the right. The chamber pressure levels for images $1-9$ are subcritical $(\sim 1.41 \mathrm{MPa})$, for 11-19 are near-critical $(\sim 3.46 \mathrm{MPa})$, and for $21-29$ are supercritical $(\sim 4.77 \mathrm{MPa})$. Inner-and outer-tube flow average temperatures at injector exit are $170 \mathrm{~K}$ and $112 \mathrm{~K}$, respectively. Davis and Chehroudi [42].

ligaments resulting from its interaction with the atomizing outer-jet flow. When the outer-jet flow rate was increased even further, finer droplets and ligaments were reported forming from the inner jet fluid, and more of its mass from the intact core was converted into droplets and ligaments. This consequently produced a shorter and less dark core for the inner jet. Moreover, as the outer flow rate increased, the heat transfer from the outer jet to the inner one intensified outside the injector, thus lowering the average density of the inner core in the injector near-field area. Under the subcritical condition, Davis and Chehroudi [42] reported that this combined outer-jet-assisted atomization of the inner jet and heat transfer processes created a dark core region more susceptible to external excitations.

4.3. Density Measurements in a Coaxial $\mathrm{LN}_{2} / G \mathrm{H}_{2} \mathrm{Jet}$. Although use of the Raman scattering is straightforward for a liquid $\mathrm{N}_{2}$ injection into a gaseous $\mathrm{N}_{2}$, in a mixture of $\mathrm{N}_{2}$ and $\mathrm{H}_{2}$ the Raman signals of the $\mathrm{N}_{2}$ and $\mathrm{H}_{2}$ are generated at two different wavelengths. Thus, in principle, utilizing an appropriate filtering technique, the signals from both species can be analyzed independently and the partial densities of each species can be determined.

This diagnostic method has been used to investigate the atomization and mixing of coaxial $\mathrm{LN}_{2} / \mathrm{H}_{2}$ injection at pressures which were supercritical with respect to the critical pressure of pure nitrogen in the inner jet; see Oschwald et al. [32]. During all the tests, the fluids were injected into a flow channel filled with $\mathrm{N}_{2}$ at near atmospheric temperature and a pressure of $4 \mathrm{MPa}\left(P_{\mathrm{r}}=P / P_{\text {crit }}=1.17\right)$. Results from a coaxial $\mathrm{LN}_{2} / \mathrm{H}_{2}$ injection are shown in Figure 19(b) and are contrasted with a single-jet case presented in Figure 19(a).
The decrease of the measured densities at large positive $r / D$ values was reported to be due to refraction of the laser beam experienced as a result of density gradients existing at the $\mathrm{H}_{2} / \mathrm{LN}_{2}$ interface. Similar radial profiles have also been obtained for a range of axial distances from the injector. Based on these data, two-dimensional species distributions were reconstructed for both $\mathrm{H}_{2}$ and $\mathrm{N}_{2}$. For the D4 test case described in Figure 19, such a distribution pertaining to the $\mathrm{H}_{2}$ is shown in Figure 20.

The maximum of the radial nitrogen density distribution at each axial distance was plotted as a function of the normalized distance $(x / D)$ from the injector; see Oschwald et al. [32]. This was then used to ascertain the evolution of the injection process for the $\mathrm{LN}_{2}$ jet downstream of the injector, as well as to assess the mixing effectiveness between the $\mathrm{H}_{2}$ and $\mathrm{N}_{2}$ species. Compared to the test case without the $\mathrm{H}_{2}$ coflow, the existence of the coflow decreased the measured nitrogen density much faster from its initial injection value near the injector exit area to a plateau which was a value for the far-field background level. The $x / D$ value at which this plateau occurred was then interpreted as representing a complete mixing of the jet with its background gas.

It was shown in Oschwald et al. [32] that when the inner-jet temperature at the injector exit was above the pseudoboiling temperature, it had a shorter decay distance $(x / D)$ than a jet with an injection temperature initially below it. In both cases, the injected nitrogen was at a supercritical pressure. They also reported that the nitrogen inner jet above the pseudoboiling temperature exhibited a gas-like density; whereas below this temperature it possessed a liquidlike density value. Interestingly, their data suggested that 


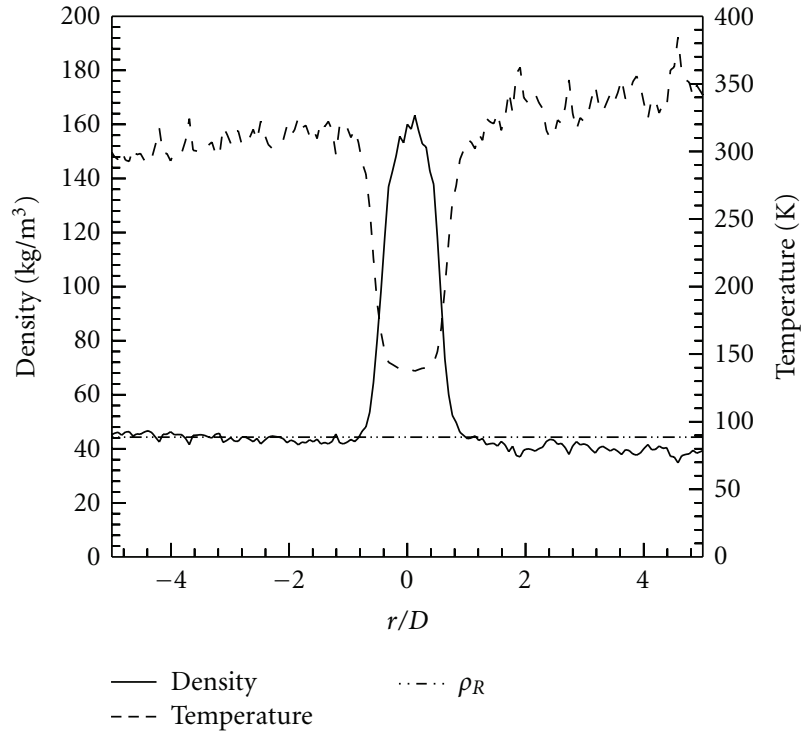

(a)

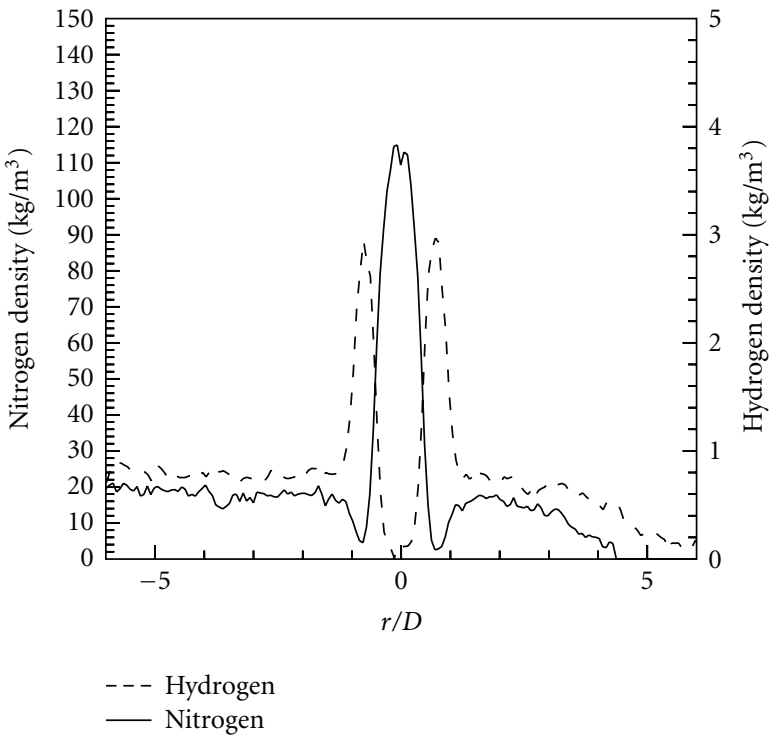

(b)

\begin{tabular}{lccccc}
\hline & Test case & $\begin{array}{c}V_{\mathrm{N}_{2}} \\
{[\mathrm{~m} / \mathrm{s}]}\end{array}$ & $\begin{array}{c}T_{\mathrm{N}_{2}} \\
{[\mathrm{~K}]}\end{array}$ & $\begin{array}{c}V_{\mathrm{H}_{2}} \\
{[\mathrm{~m} / \mathrm{s}]}\end{array}$ & $\begin{array}{c}T_{\mathrm{H}_{2}} \\
{[\mathrm{~K}]}\end{array}$ \\
\hline \multirow{2}{*}{ single $\mathrm{N}_{2}$ jet } & $\mathrm{A} 4$ & 5 & 140 & - & - \\
& $\mathrm{B} 4$ & 5 & 118 & - & - \\
\multirow{3}{*}{ coaxial $\mathrm{N}_{2} / \mathrm{H}_{2}$ jet } & $\mathrm{D} 4$ & 5 & 140 & 60 & 270 \\
& $\mathrm{E} 4$ & 5 & 118 & 120 & 270 \\
& $\mathrm{~F} 4$ & 5 & 118 & 60 & 270 \\
\hline
\end{tabular}

FIGURE 19: (a) Radial $\mathrm{N}_{2}$ density profile for test case A4 (single jet), $2 \mathrm{~mm}(x / D=1.05)$ downstream of the coaxial injector exit. (b) Radial $\mathrm{N}_{2}$ and $\mathrm{H}_{2}$ density profiles for coaxial $\mathrm{LN}_{2} / \mathrm{H}_{2}$ injection for test case $\mathrm{D} 4,2 \mathrm{~mm}(x / D=1.05)$ downstream the injector exit. Laser beam direction is from left to right. Oschwald et al. [32].

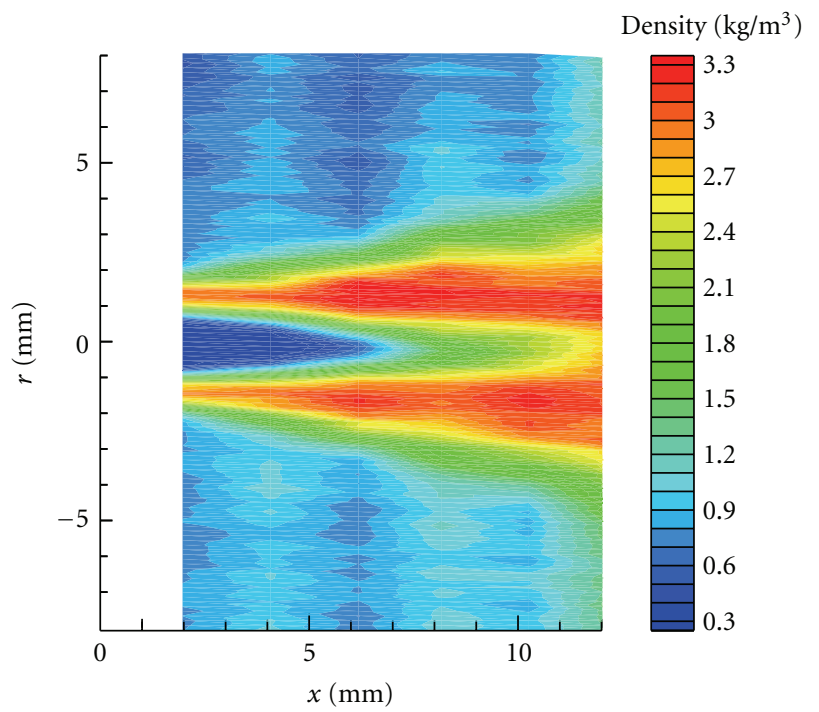

FIgURe 20: Hydrogen density for a coaxial $\mathrm{LN}_{2} / \mathrm{H}_{2}$ injection for the test case D4 in Figure $19\left(T_{\mathrm{N}_{2}}=140 \mathrm{~K}, T_{\mathrm{H}_{2}}=270 \mathrm{~K}\right)$. Oschwald et al. [32]. the effect of higher $\mathrm{H}_{2}$ velocity was found not to be as pronounced as this effect of the initial $\mathrm{N}_{2}$ density.

4.4. Spreading Angle and Core Length Measurements. Two important, and less difficult to measure, geometrical characteristics of such coaxial injectors are the spreading angle of the (combined inner and) outer jet and the length of a dark inner-core of the injected jet. They are readily determined through the high-speed shadowgraphy approach. Both the length of the dark core and the spreading angle provide good measures for mixing characteristic of the jets in coaxial injectors.

Mixing of the propellant streams, if not controlling, is at least intimately related to the combustion process. To a firstorder approximation, one measure of the mixing process is the length of the inner jet's liquid and/or dark core, which has been extensively studied by many researchers in the past. For details see Davis and Chehroudi [43]. Experimental core-length correlations, semiempirical theories, and other data for shear coaxial injector studies involving core length were competently summarized by Davis and Chehroudi [42]. Many of the correlations and semiempirical theories 
reviewed by them indicated dependencies of the core length with parameters such as outer-jet-to-inner-jet velocity ratio $\left(V_{r}\right)$, outer-jet-to-inner-jet momentum flux ratio $(M)$, density ratio $\left(\rho_{\mathrm{o}} / \rho_{i}\right)$, Reynolds' number (Re), and Weber's number (We). One difficulty Davis and Chehroudi reported with applying these relationships at supercritical pressures is that the predicted core length was very small or zero in magnitude. This is because one of the parameters in the equation was (We) and surface tension diminished greatly or vanishes all together. To overcome the difficulty in core length prediction associated with large (We) number, one group of researchers, proposed that $M$ can be used to describe the scaling of the core length for shear-coaxial injectors and need not include (We) number if it was sufficiently high; see Davis and Chehroudi $[42,43]$ for details.

But before moving forward, it should be mentioned that confusion does exist in the literature especially when vague definitions are used to characterize the core length. The terms potential-core, potential-cone, intact length, intactliquid length, and break-up length have all been used along with various measurement techniques. To be clear and to remove any possible ambiguities from the data, the darkcore length was defined as the connected dark fluid region between the injector exit area and the first break in the core as defined by an adaptive thresholding procedure; see Davis [44] or Davis and Chehroudi [42] for more details.

A plot of the dark-core length values for a coaxial injector measured by Davis and Chehroudi $[43,44]$ versus momentum flux ratio is shown in Figure 21. They used liquid nitrogen (in inner jet) with gaseous nitrogen (in outer annular jet) injected into a chamber filled with gaseous nitrogen at various pressures spanning sub- to supercritical values. The chamber temperature was always at a supercritical value. A clear distinction between the subcritical dark-core length (diamond symbols) and this length at the near-critical and supercritical chamber pressures is seen in Figure 21. Subcritical data suggests a much longer length than that at supercritical pressures for a given momentum flux ratio. Davis and Chehroudi indicated that both the near-critical and supercritical pressure conditions produced an appearance of a single-phase coaxial jet even though liquid nitrogen was injected through the inner tube of the coaxial jet. Recall that earlier in the single-jet part of this paper, Chehroudi and his coworkers $[5,10,26]$ also found that two-phase-appearing jets were solely observed at subcritical chamber pressures. However, at supercritical chamber pressures, the spreading rate and fractal dimension values were the same as those seen for gaseous jets injected into a gaseous environment (i.e., single phase); that is, these supercritical single jets can be considered as variable-density, single-phase, gaseous jets. This conclusion presented earlier for the single-jet injection is something Davis and Chehroudi $[43,44]$ also showed to be mostly valid when it comes to the dark-core length observations in coaxial jet injectors. Evidences in support of such a behavior for coaxial injectors are presented in the next few paragraphs.

The dashed line in Figure 21 is a least-square curve fit to the subcritical data, and the dotted line is a similar

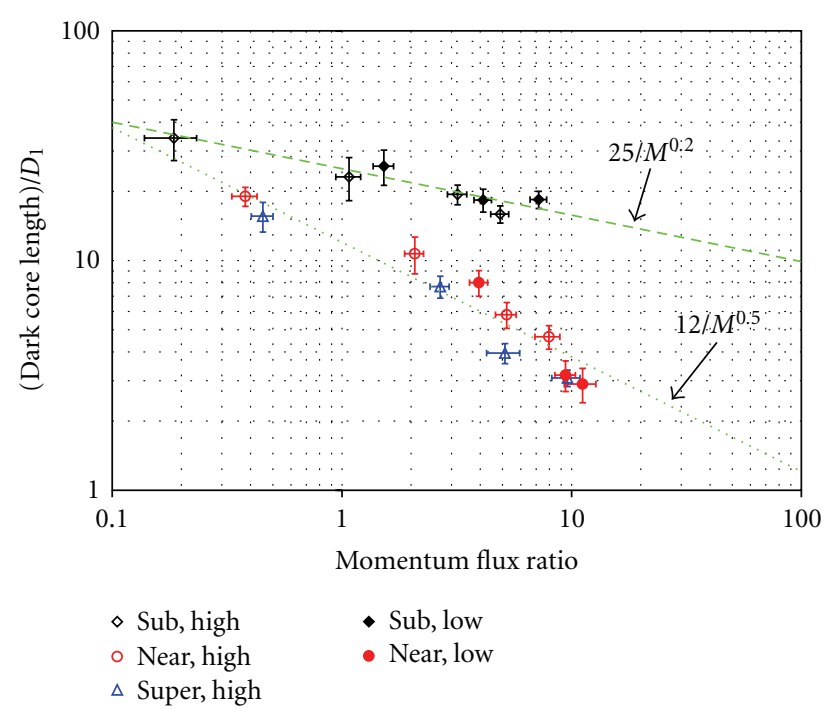

FIGURE 21: Plots of the dark-core length versus momentum flux ratio for a coaxial injector. Liquid nitrogen (inner jet) and gaseous nitrogen (outer annular jet) injected into chamber filled with gaseous nitrogen. The diamond, circle, and up-triangle symbols represent sub-, near-, and supercritical chamber pressure, respectively. The hollow symbols are at a "high" outer-jet temperature $(\sim 190 \mathrm{~K})$ and solid symbols are at a "low" outer-jet temperature $(\sim 140 \mathrm{~K})$. The dashed line is $25 / M^{0.2}$ and the dotted line is $12 / M^{0.5}$. Davis and Chehroudi $[43,44]$.

fit to the near-critical and supercritical data. As indicated by the equations in this figure from Davis and Chehroudi $[42,43]$, the single-phase-appearing (i.e., at near-critical and supercritical pressures) data has a $M^{-0.5}$ dependence on momentum flux ratio. However, they indicated that the twophase-appearing subcritical data had a weaker dependence, $M^{-0.2}$, than the single-phase dark-core length at nearand super-critical chamber pressures. Other quantitative differences between the subcritical and supercritical cases have been reported before. They also reported that the $M^{-0.5}$ form of the dependency on $M$ parameter under near- and supercritical pressures is not only valid for gas-gas shearcoaxial jets, but is also for any single-phase (gas-gas or liquidliquid) shear-coaxial jet.

Figure 22 was constructed by Davis and Chehroudi $[42,43]$ aiming at a comprehensive comparison of the dark-core length they measured for the $\mathrm{LN}_{2} / \mathrm{GN}_{2}$ with all other comparable single-phase and two-phase data available in the literature for the potential-core length, intact-core length, and break-up length. This unique figure represents all relevant data in the literature concerning core length spanning 5 orders of magnitude in momentum flux ratio. They also noted that as $M$ approaches zero, one reaches a limit which defines a single round jet configuration because the outer-jet velocity becomes zero. From the information shown in Figure 22, it seems that for $M<1$, the data points converge and approach the core length range expected for single round jets reported by Chehroudi et al. [27] and Oschwald et al. [45]. The single-phase data by others (i.e., injectants and chamber content all are in one phase, either all gaseous or all liquids) along with the Davis and Chehroudi's 


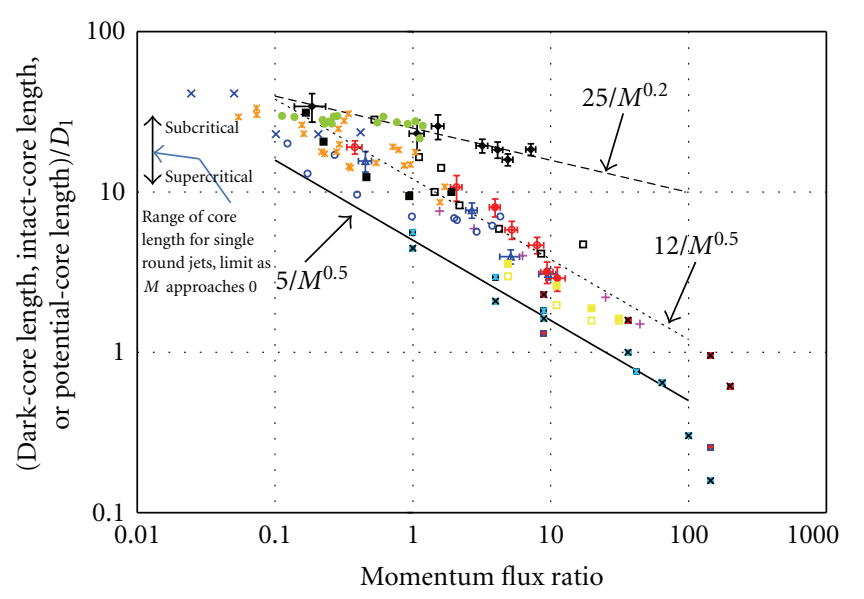

- Subcritical $P$; high outer $T(*)$

$\triangle$ Supercritical $P$; high outer $T\left({ }^{*}\right)$

- Near-critical $P$; low outer $T(*)$

- Near-critical $P$; high outer $T$ (*)

- Subcritical $P$; low outer $T$ (*)

- Eroglu et al. Re $=4370$

* Favre-Marinet $\mathrm{DR}=0.138$ air

- Favre-Marinet DR $=0.138 \mathrm{He}$

- Favre-Marinet DR $=0.655$ air

* Favre-Marinet $\mathrm{DR}=0.028 \mathrm{He}$

Rehab et al. $D 3 / D 1=1.37$

+ Au and Ko

- Woodward KI (aq.)-He

* Woodward KI (aq)- $\mathrm{N}_{2}$

- Eroglu et al. $\mathrm{Re}=1456$

$\times$ Eroglu et al. Re $=9328$

- Rehab et al. $D 3 / D 1=2.29$

- Englebert et al.

FIgURE 22: This figure shows comparison of the present coaxialjet dark-core length measurements with all other relevant core length data available in the literature versus momentum flux ratio. Amongst the data reported by others, Eroglu et al. [47], Englebert et al. [48], and Woodward [50] are two-phase flows and the rest are single phase. The range of core length for cryogenic single jet $\left(\mathrm{LN}_{2} / \mathrm{GN}_{2}\right)$ is also shown at the left margin. Davis and Chehroudi $[42,43]$.

$\mathrm{LN}_{2} / \mathrm{GN}_{2}$ supercritical results are all seen in Figure 22 to follow a dependence close to $L / D_{1}=A M^{-0.5}$, where the constant $A$ is between 5 and 12. This information combined with the clear separation of all the two-phase data, including the subcritical core length data seen in Figure 22, essentially suggests a two-phase behavior at subcritical and a singlephase conformance at supercritical chamber pressures.

Even though best efforts were made to use the most relevant data by other investigators, some shortcomings were reported. For example, at $M>100$ in Figure 22, the experiments of Favre-Marinet and Camano Shettini [46] exhibited a recirculation bubble at the end of the core, and thus the core length decreases as depicted in this figure. The injectors used to produce the two-phase coaxial jets of Eroglu et al. [47] and Englebert et al. [48] had apparently much larger outer-jet gap widths than what is typical of rocket injectors. Additionally, the apparatus of Eroglu et al. [47], reported in Faragó and Chigier [49], did not produce fully turbulent inner jet until $\operatorname{Re}>10^{4}$. Davis and Chehroudi $[42,43]$ explained that the lack of a fully turbulent inner jet and the significant differences between their injector and shear-coaxial ones used in rockets could be the reason why the core length measured by Eroglu et al. [47] is shorter than those observed in their work. Englebert et al. [48] reported that the core length scaled with $M^{-0.3}$. The two-phase core length by Woodward [50] for the water potassium iodide solution with helium, however, obeyed very nearly the trends for the subcritical data points (i.e., $25 M^{-0.2}$ ). Considering that the momentum flux ratios near or higher than 10 are of importance for liquid rocket engines (LRE), Davis and Chehroudi $[42,43]$ indicated that the data for the subcritical (two-phase) case was the only reported information in the neighborhood of the $M=10$.

Outer-to-inner jet velocity ratio, $V_{r}$, has been a design parameter for shear-coaxial injectors, particularly, as a criterion to ensure stable operation of liquid rocket engines (LRE). For $\mathrm{LOX} / \mathrm{H}_{2}$ engines, the design rule of thumb has been to keep the velocity ratio greater than about 10 in order to maintain stable engine operation, as is discussed by Hulka and Hutt [40]. Although this stability criterion has been suggested by the experimental data, no physical explanation has been provided. Related to this is a method to rate a LRE for combustion instability, known as the temperature ramping. The temperature ramping of a fired $\mathrm{H}_{2} / \mathrm{O}_{2} \mathrm{LRE}$ is accomplished by lowering the temperature of the $\mathrm{H}_{2}$ (in the outer jet of the coaxial injector) while maintaining its mass flow rate at a constant value. The lower the $\mathrm{H}_{2}$ temperature when the onset of the combustion instability is detected, the broader the stability margin of that particular LRE. The temperature ramping is related to and affects the velocity ratio. This is because one of the outcomes of lowering $\mathrm{H}_{2}$ temperature at constant mass flow rate is the increased density and consequently, lowered injection velocity values. Davis and Chehroudi $[42,43]$ therefore investigated the impact of the velocity ratio on their nonreacting $\mathrm{LN}_{2} / \mathrm{GN}_{2}$ coaxial jets, hoping that results may shed some light on the effects of velocity ratio observed in fired production engines. This is explained next.

To determine the effects of the outer-jet temperature (which is $\mathrm{GN}_{2}$ in Davis and Chehroudi's work) on the coaxial jet, Davis and Chehroudi [42, 43] studied two nominal temperatures of $\sim 190 \mathrm{~K}$ and $\sim 140 \mathrm{~K}$, called "high" and "low," respectively. Recognizing that the dark-core length is a dynamic quantity, its basic characterization required measurements of both the average and root mean square (RMS) of length fluctuations. The averaged dark-core lengths were then measured and are shown in Figures 23(a) and 24 (a) as a function of velocity ratio for sub-, near-, and super-critical chamber pressures. The RMS of the variations of the dark-core length is also shown in Figures 23(b) and 24(b). Note that Figures 23 and 24 present results for conditions when the external acoustic field is both turned on and off for "high" and "low" outer-jet temperatures. However, it is important to emphasize that in this section only the results when the acoustic field is turned off are discussed, deferring the impact of the externally imposed acoustic field to the next part of this paper. 


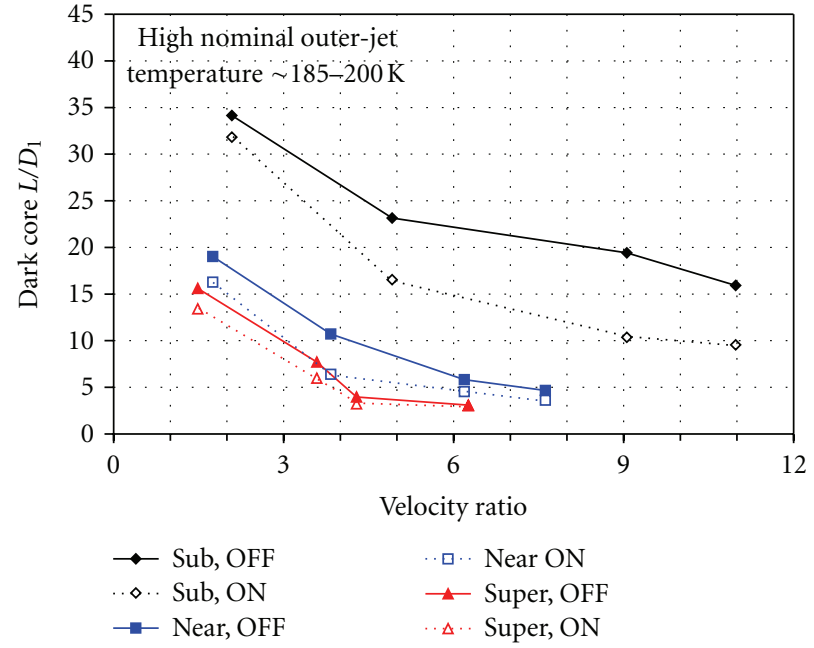

(a)

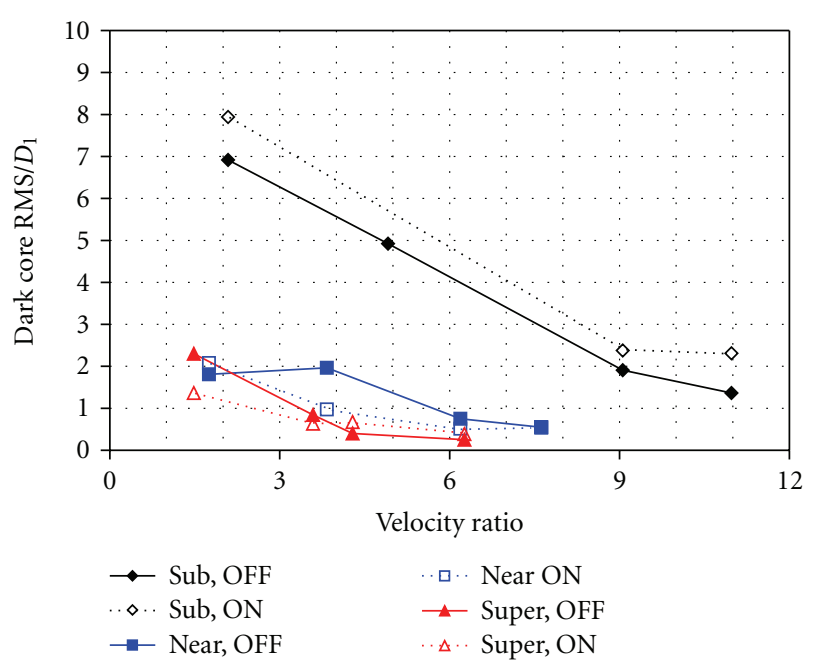

(b)

FIGURE 23: Plot of the averaged dark-core length (a) and the root mean square (RMS) of the length variations (b) normalized by the inner diameter of a $\mathrm{LN}_{2} / \mathrm{GN}_{2}$ coaxial jets injector. The solid symbols and lines represent the data when the acoustic driver is off, and the hollow symbols and dotted lines show the data when the acoustic driver was operated at $\sim 3 \mathrm{kHz}$. The diamond, square, and up-triangle symbols are sub-, near-, and supercritical chamber pressures, respectively. All cases are for the high nominal outer-jet temperature of $\sim 190 \mathrm{~K}$. In the inset, the words sub, near, and super refer to subcritical, near critical, and supercritical chamber pressures, respectively, and the words OFF and $\mathrm{ON}$ refer to the acoustic driver being off and on at $\sim 3 \mathrm{kHz}$, respectively. Davis and Chehroudi $[42,43]$.

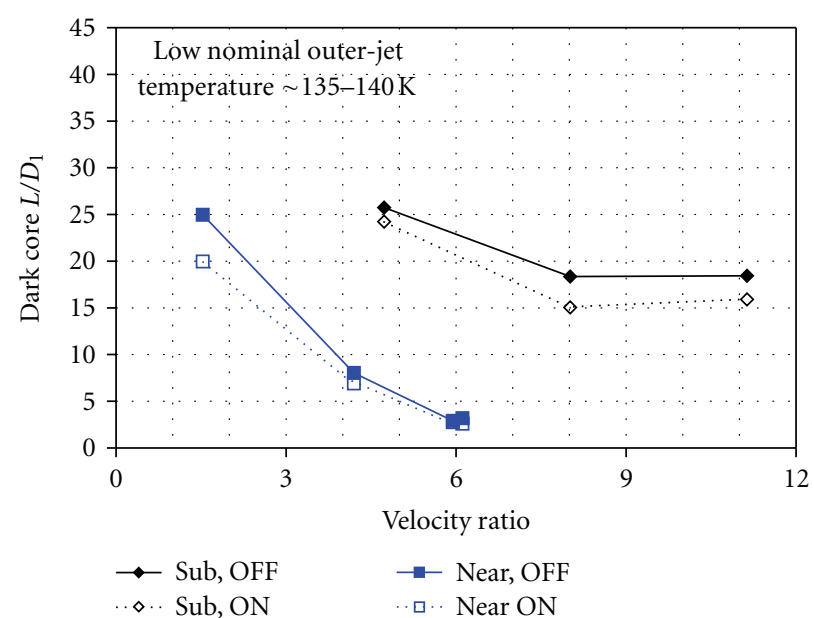

(a)

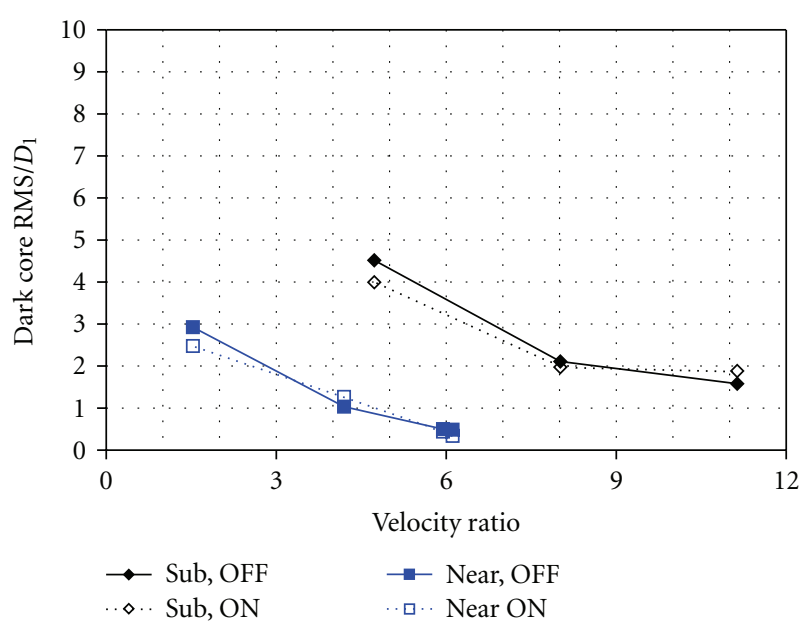

(b)

FIgURE 24: Plot of the averaged dark-core length (a) and the root mean square (RMS) of the length variations (b) normalized by the inner diameter of a $\mathrm{LN}_{2} / \mathrm{GN}_{2}$ coaxial jets injector. The solid symbols and lines represent data when the acoustic driver is off, and the hollow symbols and dotted lines show results when the acoustic driver is operated at $\sim 3 \mathrm{kHz}$. The diamond and square symbols are for sub- and near-critical chamber pressures, respectively. All cases are for the low nominal outer-jet temperature of $\sim 140 \mathrm{~K}$. In the inset, the words sub and near refer to subcritical and near-critical chamber pressures, respectively, and the words OFF and ON refer to the acoustic driver being off and on at $\sim 3 \mathrm{kHz}$, respectively. Davis and Chehroudi $[42,43]$.

Evident in Figures 23(a) and 24(a) is the fact that the length of the dark core decreases as the chamber pressure is increased from sub- to super-critical conditions. The dark core provides an indication of high-density regions of the flow. At a given fixed chamber pressure, as $V_{r}$ is increased, the length of the dark core decreases and appears approaching a constant value. The RMS values of the dark-core length fluctuations, shown in Figures 23(b) and 24(b), exhibit somewhat similar trends to those seen for the averaged values, that is, substantially lowered at near- and supercritical chamber pressures.

It is known that for a liquid-fueled rocket, atomization and break-up processes, interactions between the propellant jets, droplet formation, and vaporization are all affected by the pressure and, particularly, velocity fluctuations. Also, for any chemically reacting system, it is expected that, to certain 
but different degrees, the rate at which energy is released be sensitive to the rate of changes in temperature, density, pressure, and, of course, particularly mixture ratio. It is then intuitive to relate, in some form, the RMS values of the dark-core length fluctuations to mixture ratio variations. On the other hand, a low RMS value can be interpreted as the coaxial jet's "inherent steadiness" (or insensitivity to external stimuli) and vice versa. Examination of Figures 23(b) and 24(b) clearly shows that this property is drastically reduced as the velocity ratio is increased. Although these results are for one injector (i.e., no interinjector interaction), Davis and Chehroudi [42, 43] proposed a linkage between these nonreacting results and production-engine combustion instabilities observed at low velocity ratios. They stated that "it is then quite possible that the observed improvement in combustion stability at higher values of velocity ratio is a result of the inability of the jet to generate large mass flow rate fluctuations under these conditions, thus weakening a key feedback mechanism for the self-excitation process."

As indicated earlier, in temperature ramping exercises for stability rating of $\mathrm{LOX} / \mathrm{H}_{2}$ engines, the mass flow rate of the outer-jet hydrogen is maintained at a constant value. Davis and Chehroudi $[42,43]$ also attempted to provide a linkage between the temperature ramping and their experimental data. They said that as the temperature of the $\mathrm{H}_{2}$ is decreased during a ramping episode, the $\mathrm{H}_{2}$ becomes more dense, which decreases the injector velocity ratio at a constant mass flow rate. The RMS plots shown in Figures 23(b) and 24(b) suggest that such a decline in this ratio amplifies the jet's inherent unsteadiness (i.e., higher RMS values), providing a possible explanation for the engine's eventual arrival into an unstable zone as a temperature ramping test proceeds.

On the spreading angle or growth rate of the coaxial jets, Figure 25 is a compilation of experimental measurements reported earlier by Chehroudi et al. $[5,10]$ on single jets along with those newly acquired from a coaxial injector and other data. The tangent of the total jet divergence angle is plotted against the chamber-to-injectant (for single jets) or chamber-to-inner-jet (for coaxial jets) density ratio. The data taken by Chehroudi for single jets in the same facility is marked by $(*)$ in the legend. Note that there are also some theoretical expressions presented in this figure for comparison purposes. The coaxial injector data follows the direction of the arrow as chamber pressure is changed from sub- to near- and to super-critical condition. Interestingly, the data for coaxial injection at subcritical condition conforms with the classical incompressible variabledensity shear layer (Dimotakis' theory) which is also what is observed for the single jet, but at supercritical chamber pressures. This suggests that under such a condition the outer jet in Chehroudi's coaxial injector grows similar to a variabledensity gaseous jet. Coaxial data, although stays nearly at a constant value, departs from the rest in this figure as chamber pressure is raised towards the supercritical condition.

\section{Coaxial Jets with External Excitation}

Cold flow studies investigating the interaction of acoustic waves with single-jet injectors have recently been extended

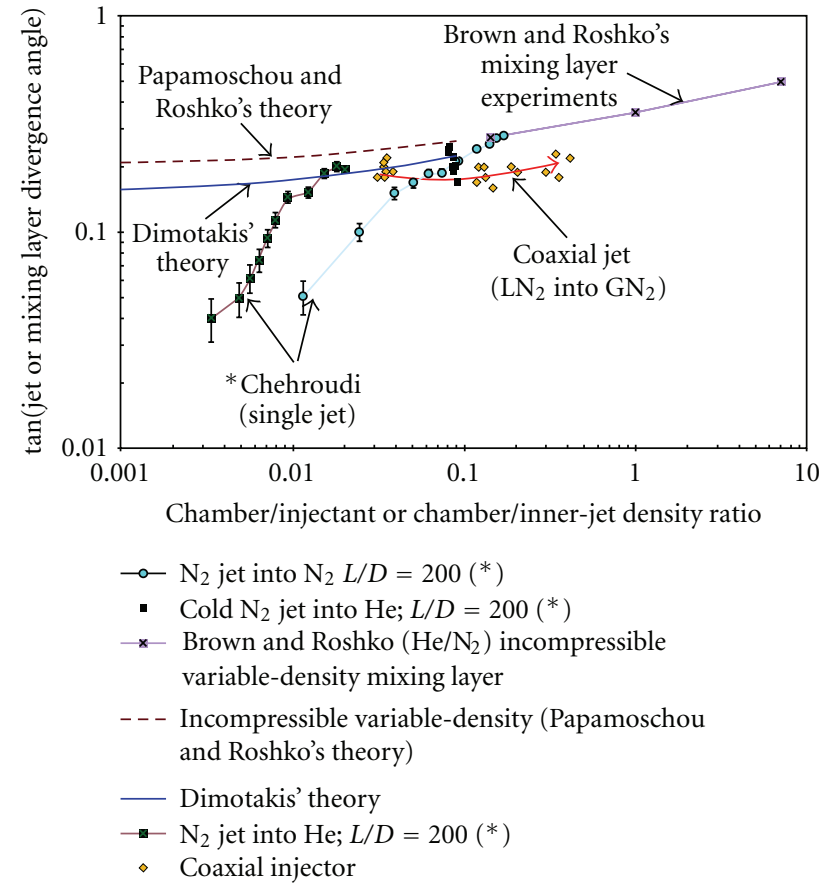

FIGURE 25: Spreading rate of the shear layer versus the chamber/injectant or chamber/inner-jet density ratio for single and coaxial jets compared with different predictions for planar shear layers. For the coaxial data, the chamber pressure increases from sub- to near- and to supercritical conditions in the direction of the arrow.

to include coaxial injectors by Chehroudi et al. [51], Davis and Chehroudi [42] and Leyva et al. [52]. Their objective was to characterize the coaxial jets response and understand how nonreacting injector results are related to historical liquid rocket engine combustion instability data. As it will be shown in the next few paragraphs, the aforementioned observation that the single-jet interaction with the externally imposed acoustic waves was stronger at subcritical pressures than at supercritical pressures appears to remain generally true for the coaxial-jet injector as well, although some influences of the mass flow ratio and initial temperature were observed. Efforts by Chehroudi's group continue in order to better understand how various parameters control the coupling mechanisms.

Figure 26, taken by Davis and Chehroudi [42], shows a sample of ten consecutive images at sub- (rows one and two from top), near- (rows three and four), and supercritical (rows five and six) chamber pressures from a $\mathrm{LN}_{2} / \mathrm{GN}_{2}$ coaxial injector. Rows one, three, and five are when the acoustic driver was off and the remaining ones are when it was activated at $\sim 3 \mathrm{kHz}$. The evolution of the jet in time is from left to right in this figure and the time interval between frames is about $55.6 \mu \mathrm{s}$. Prominent in all images of the jet is the existence of a dark central region. After close examination of many images at different magnifications, Davis and Chehroudi [42] reported that the dark core under the unexcited subcritical pressures (Figure 25, row one) can be approximated as a cylinder-like structure with unstable 


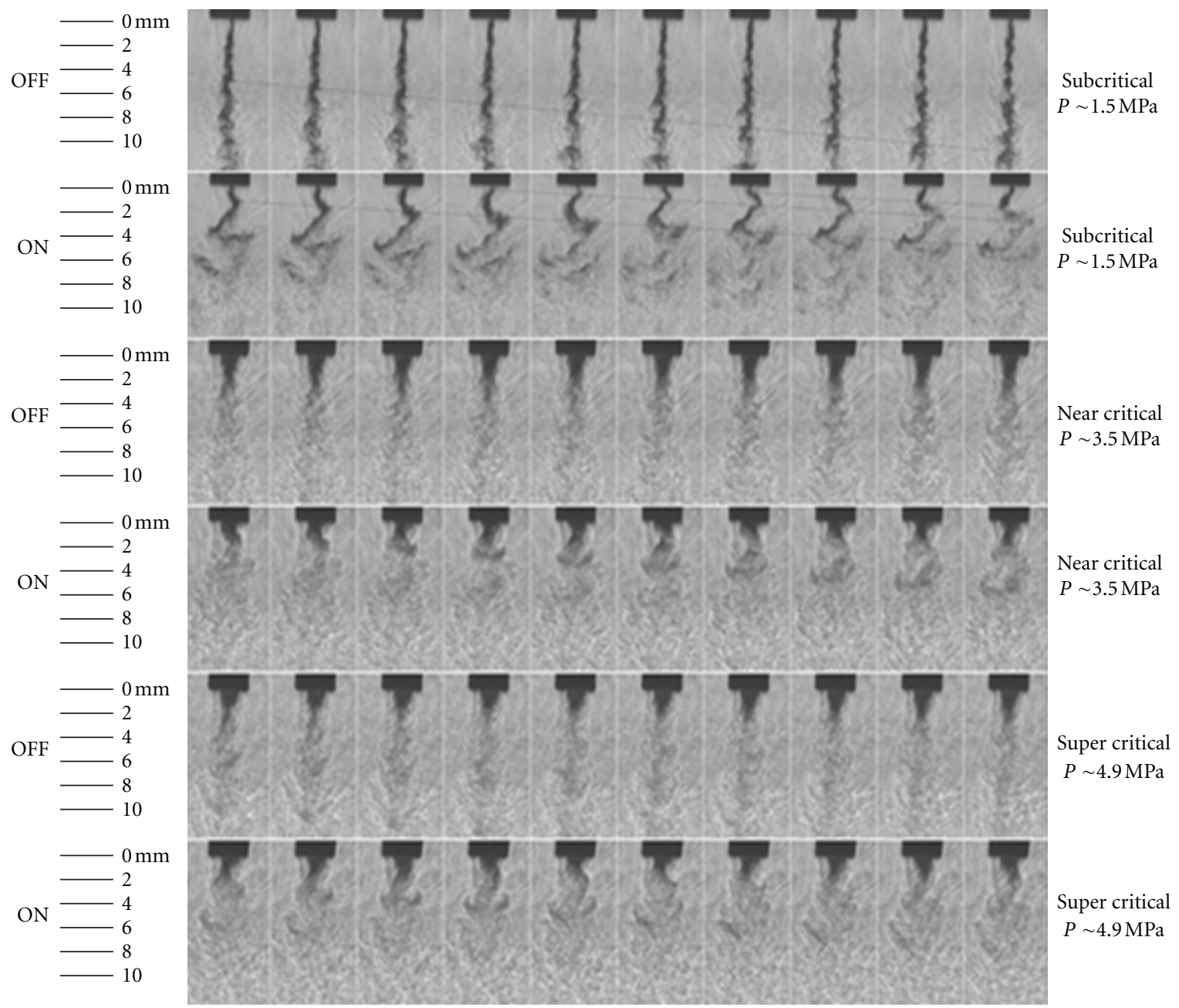

Figure 26: Consecutive frames from high-speed shadowgraph movies or a coaxial injector $\left(\mathrm{LN}_{2} / \mathrm{GN}_{2}\right.$ into $\mathrm{N}_{2}$-filled chamber) with the acoustic driver turned off (in rows 1, 3, and 5) and on (in rows 2, 4, and 6) at $\sim 3 \mathrm{kHz}$. Time increases from left to right with an interval of 55.6 ms between frames. The first two rows are at a subcritical chamber pressure $(\sim 1.5 \mathrm{MPa})$, the third and fourth rows are at a near-critical chamber pressure $(\sim 3.5 \mathrm{MPa})$, and the fifth and sixth rows are at a supercritical chamber pressure $(\sim 4.9 \mathrm{MPa})$. The acoustic driver is turned off for the first, third, and fifth rows and on for the second, fourth, and sixth at $\sim 3 \mathrm{kHz}$. The light gray lines in the first and second rows connect fluid structure as they evolve in time. Davis and Chehroudi [42].

surface waves of low amplitudes. However, when they increased the chamber pressure to near- and supercritical pressures (rows three and five), the dark-core length became shorter (as indicated earlier) and the structure changed to a more conical shape near the injector exit area. The conical structure of the dark core has been reported before for singlephase coaxial jets by Lasheras and Hopfinger [53] but not at supercritical conditions.

Excitation of the jet with an acoustic driver yielded significantly different behavior of the dark core compared to that of the unexcited one. The strongest effect was observed under subcritical pressures and made clear in Figure 26. Note that the direction of the acoustically induced velocity oscillations is horizontal (towards left and right) in images presented in Figure 26. It is seen that the core of the jet forms large-scale sinusoidal structures as a result of this imposed velocity field oscillations. From the movies they acquired, Davis and Chehroudi $[42,43]$ explained an observed dominant pattern which is also shown in Figure 26. They said that as a piece of fluid left the injector tip, the momentum from the acoustically induced motion caused a transverse displacement pushing the core of the jet into the higher-speed annular jet area. Then the dense fluid from the core experienced acceleration in the axial direction, which was caused by the high-speed annular jet motion. Upon reversal of the acoustic field, the dense fluid, which was initially from the core but now in the high-speed annular flow, appeared to maintain its transverse component of the momentum imparted upon leaving the injector and hence the dense fluid particle did not reverse its direction. The dense fluid parcel then slowed (both in the axial and transverse directions) as it arrived at the shear layer between the outer jet and the chamber fluid farther downstream, where a "cusp-shaped" structure was formed from the dense 


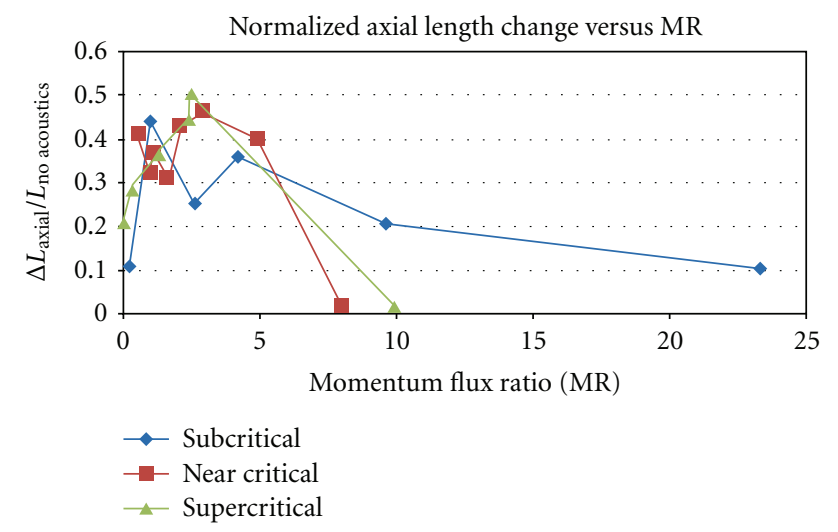

FIGURE 27: Maximum length change between dark-core length without acoustics and dark-core length with acoustics $\left(\Delta L_{\text {axial }}\right)$ divided by the dark-core length without acoustics $\left(L_{\text {no acoustics }}\right)$ for each momentum flux ratio case. Results for a coaxial injector. Rodriguez et al. [54].

fluid originating in the core of the jet. Subsequent mixing and heat transfer from the outer-jet to the inner-jet core fluid ultimately cause the fluid parcel to be indistinguishable from the outer-jet fluid.

Figures 23(a) and 24(a) also show the impact of the outer-jet-to-inner-jet velocity ratio $\left(V_{r}\right)$ on the mean darkcore length at three different chamber pressures but under externally imposed acoustic excitation. On an average, when the dark core feels the imposed external acoustic field, its length is shorter than or equal to that when the acoustic driver is turned off. At the near-critical and supercritical chamber pressures, as the $V_{r}$ increases, the difference between the lengths of the dark core, measured with and without the acoustic field, diminishes. One distinct feature of plots in Figures 23(b) and 24(b) is that, at any given velocity ratio, the RMS of the core fluctuations under subcritical condition is much higher than near- and, particularly, super-critical conditions. And equally important, is that this statement is true with or without the externally imposed acoustic excitation field. Using Davis and Chehroudi's $[42,43]$ interpretation of the RMS, it appears that the jets under supercritical conditions are more inherently insensitive to their environment even when an acoustic field is imposed. Another important feature here is the fact that the magnitude of this RMS of fluctuations drastically declines for all cases, more so for the subcritical case, when velocity ratio is increased. Implications of these unique and important observations for the combustion instability in liquid rocket engines are provided later in this writing.

Rodriguez et al. [54] initiated a set of experiments to analyze the impact of the transverse acoustic wave phase variations on the magnitude of the inner-jet dark-core length. The chosen coaxial jet was exposed to different acoustic conditions by varying the phase between two acoustic sources which faced each other while the jet was located at the midpoint between the two sources, all being inside a high-pressure chamber. The coaxial jet was exposed to a subcritical and a near-critical pressure environment. The measurements were performed on backlit images of the coaxial jet obtained with a high-speed camera. The outerjet-to-inner-jet momentum flux ratio was varied from 1 to 20 for subcritical conditions and from 0.6 to 5 for nearcritical conditions. The resonant frequency of the system was approximately $3 \mathrm{kHz}$ and the maximum pressure variation with respect to total pressure was 3\%. Figure 27 shows summary of their results. This figure presents the maximum dark-core length reduction between the case without the external acoustics and that with the acoustics for all phase angles, each normalized with the core length without the acoustics and then plotted as a function of $M$ (momentum flux ratio). The analysis of this data showed a reduction of this length with acoustic excitation within the momentum flux ratio $(M)$ range between 1 and 5 for all chamber pressures, except at the subcritical which extended up to $M$ value of 10 . The effect of the acoustic waves at lower or higher $M$ values did not have significant influences on the behavior of the dark-core length.

Rodriguez [55] also examined effects of the injector geometry, specifically the impact of the inner-tube wall thickness on the jet behavior and its interaction with the acoustic field. A new injector design was considered with a thinner inner-tube wall thickness value which consequently produced an inner jet with larger diameter than the original one used in earlier studies. The highest reduction in the dark-core length for the tests with the new coaxial injector geometry took place at moderate $M$ values which supports similar evidence obtained with the original injector possessing a larger inner-tube wall thickness. The results for the new injector also showed that for a given outer-to-inner jet momentum flux ratio, the normalized values of the dark core length between subcritical and nearcritical cases agree reasonably well.

\section{Impinging Jets}

To the best of this author's knowledge, there is only one impinging jet data set reported under both sub- and supercritical conditions; see Chehroudi [56]. However, the study conducted by Anderson et al. [57] is an example at 


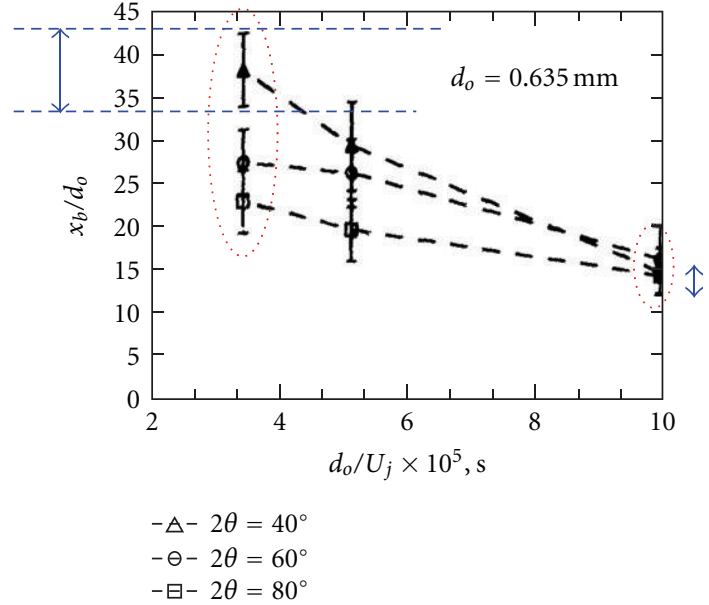

FIGURE 28: This figure shows sheet break-up length for an impinging injector as a function of instability parameter at three different impingement included angles. Much higher sensitivity of the sheet break-up length is seen with included angle $(2 \theta)$ at low $d n / V$ $\left(=d_{o} / U_{j}\right.$, in the original article) values. Anderson et al. [57].

high-pressure subcritical chamber conditions which is of relevance to the discussion in this section.

Figures 28 and 29 taken from Anderson et al.'s [57] studies show measured data for the sheet break-up length in a doublet impinging jet injector design plotted against injector-hole-diameter $(d n)$-to-injection-velocity $(V)$ ratio, that is, $d n / V$. The $d n / V$ ratio is also called the stability parameter. This is because of the dependency of the rocket engine stability region (using this type of injector design) with the $d n / V$ ratio. Large differences between the sheet break-up lengths for different pressures and different impinging-jet included angles $(2 \theta)$ at low values of the stability parameter are seen in Figure 29. For example, Figure 29 strongly suggests higher sensitivity of the injector when $d n / V$ is reduced through an increase in $V$ (injection velocity) values. This is simply deduced by the enlarged size of the scatter bounds at any given pressure and sensitivity to pressure changes at low $d n / V$ values. Although strictly speaking one should have its frequency response (amplitude and phase) measured, Chehroudi [56] interpreted these results as an indication of injector hypersensitivity under such conditions. At a given pressure or included angle, the data scatter band shown in Figures 28 and 29 is also largest at low $d n / V$ values, again and consistently suggesting a more erratic/chaotic dynamic behavior.

The changes in the dark-core (break-up) length were also reported in Figure 30 by Chehroudi [56], showing a progressive increase in chamber pressure up to a supercritical condition for liquid nitrogen injection into gaseous nitrogen environment with no externally imposed acoustic field. The long preimpingement length seen along the jet is expected due to $L / d n$ of about 100 which was intentionally designed to obtain a fully developed condition at the hole exit plane and also to accentuate the effects of chamber pressure on the nature of the impingement. Obviously, shorter dark core

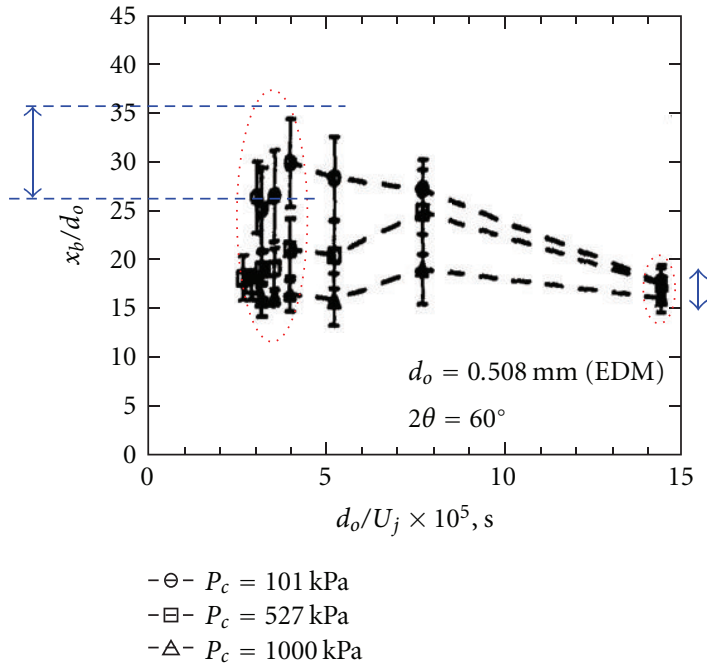

FIGURE 29: This figure shows sheet break-up length for an impinging injector as a function of instability parameter at three different chamber pressures. Much higher sensitivity of the sheet break-up length is seen with chamber pressure at low $d n / V\left(=d_{o} / U_{j}\right.$, in the original article) values. Anderson et al. [57].

is achieved for lower (injector hole) $L / d n$ values used in LRE. It is observed that not only the dark-core length of each individual jet is reduced as supercritical pressures are approached (as before and as expected), but the jet also thickens during this process. Clearly each jet is going through a very similar process discussed earlier in the context of single jet discussion. However, there are important implications as a result of such anatomical changes in an impinging injector which will briefly be discussed in the final part of this paper.

Aside from these results, the author is not aware of any published impinging jet data of relevance under supercritical chamber conditions. The situation is even worse as no systematic investigation or results are available on the impact of external excitation (imposed acoustic field) on impingingjet injectors.

\section{Implications for Combustion Instability}

From the review of recent studies presented here, it is clear that our understanding of the jet behavior under transcritical and supercritical conditions has substantially improved in the past 20 years primarily due to pioneering experimental studies reviewed here. Computer simulations have also contributed heavily in the understanding and design process but are not within the scope of this short review. While experimental results have been very valuable for simulations and provided physics-based improvements in computational efforts, the implications of these results towards formulating "a big-picture" and efforts in constructing such an image through synergies amongst these pieces of information were needed and are just beginning. One relevant sketch of this big picture this author knows is that attempted by Chehroudi [56]. He, in his work, has made an effort to collect pieces of experimental information and offered a compelling 


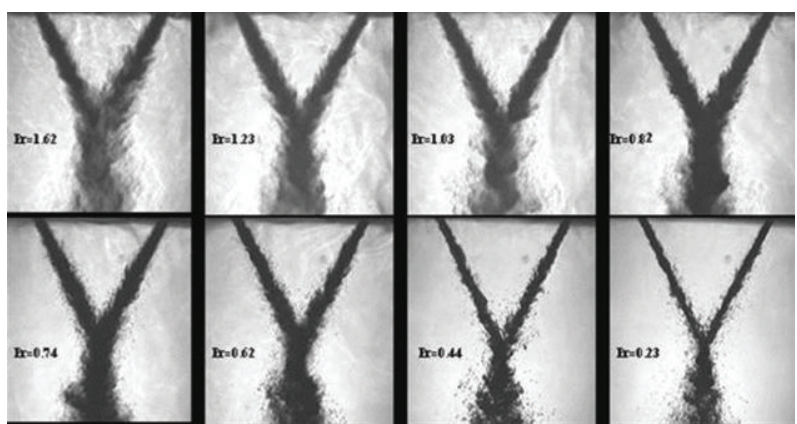

(a)

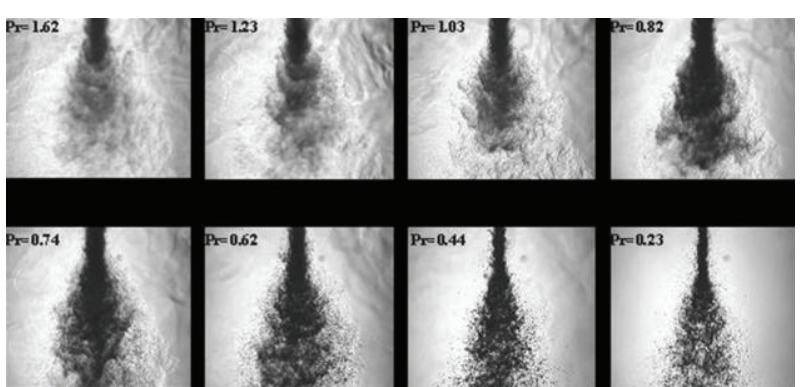

(b)

FIGURE 30: Instant images of sub-, near-, and supercritical impinging jets for $\mathrm{LN}_{2}$ into $\mathrm{GN}_{2}$ (room temperature) injection by Chehroudi. The last two rows show the same injector in the first two rows but viewed at a 90-degree angle. $P_{\mathrm{ch}}=0.8,1.5,2.1,2.5,2.8,3.5,4.2,5.5 \mathrm{MPa}$; from lower right to upper left, $P_{\mathrm{ch}}=100,200,300,350,400,500,600,800$ psig. For nitrogen: $P_{\text {critical }}=3.39 \mathrm{MPa} ; T_{\text {critical }}=126.2 \mathrm{~K}$. Re $=25,000$ to 70,000; holes' length-to-diameter ( $L / d n)$ of $\sim 100$; no cavitation; injection velocity: 10-15 m/s. Chehroudi [56].

argument to link nonreacting cold-flow studies (in test rigs) to fired subscale engine information and to what occurs in production engine in the context of combustion instability. Of course, as the history of scientific discovery testifies, such efforts are mostly evolutionary in nature and, while they approach full understanding in time, are incomplete in every step taken. Nevertheless, they are important in order to continuously improve the "sketch" and hopefully soon converge to a colorful big picture. Therefore, in this section, the work by Chehroudi [56] published in such a context is summarized to present a sample of an important attempt to tackle the problem through placement of pieces of jigsaw puzzle. But before doing so, a concise description of the combustion instability in liquid rocket engine is offered to provide a context and sufficient background for Chehroudi's work.

Acoustic combustion instability has been one of the most complex phenomena in liquid rocket engines and therefore difficult to fully understand, control, and predict particularly in the design of high-power rockets. The difficulty arises from the emergence of oscillatory combustion with rapidly increasing and large pressure amplitudes. This leads to local burnout of the combustion chamber walls and injector plates which is caused through extreme heat-transfer rates by high-frequency pressure and gas velocity fluctuations; see Harrje and Reardon [58] and Yang and Anderson [59]. It is thought that resonance acoustic modes of the thrust chamber, amongst them the transverse modes being the most troublesome, are excited through the energy provided by the combustion. The amplification process is thought to include a feedback of information from the acoustic field to the injector or near-injector phenomena which in turn tends to reinforce the combustion-to-acoustic-field energy transfer processes.

This energy transfer reasoning alone is the widely cited general principle by Lord Rayleigh [60]. In essence, he made a phasing argument and stated that the interaction between the combustion heat release and the acoustic field is the strongest if heat is added in a region of space and at the time when the acoustic amplitude is the highest.
Although this view has been useful to understand a part of the big picture, evidences gathered by past investigations attributed combustion instability to a complex interaction of the external acoustic field with the fuel injection or near-injector processes as a feedback mechanism, thereby leading to incidences of instability in rocket engines. See, for example, Heidemann and Groeneweg [61], Anderson et al. [57], and Hulka and Hutt [40]. For this and other reasons, controlled studies have been conducted probing into the effects of acoustic waves on gaseous and liquid jets from a variety of injector hole designs. A series of investigations concentrated on disturbances induced from within the injection system. They considered the effects of acoustic fields on many phenomena such as flow structure, vortex pairing, and shear layer growth rate in the initial region of the jet (e.g., see a short review article by Kiwata et al. [62]). More relevant to the work reported here are a few reports and articles on gaseous and (in particular) liquid jets under the influence of external (transverse and longitudinal) acoustic fields. The experimental work under high-pressure supercritical condition has already been reviewed here. More information on this and others can be found in Chehroudi and Tally [39] and Davis and Chehroudi [42, 43].

In Davis and Chehroudi's $[63,64]$ experimental work on a cryogenic nonfired coaxial injector at sub- and supercritical pressures, they have offered a plausible explanation of why in temperature ramping stability rating exercises an engine becomes unstable. In such tests, which are usually conducted in $\mathrm{LOX} / \mathrm{H}_{2}$ cryogenic liquid rocket engines (LRE), they proposed that a progressive reduction of the propellant $\left(\mathrm{H}_{2}\right)$ temperature decreases the outer-to-inner jet velocity ratio for shear coaxial injectors and therefore pushes the engine into an unstable operating zone. As indicated earlier, this velocity ratio was found to be a key parameter defining the stability of the engine; see Hulka and Hutt [40]. But what this velocity ratio does to the jet itself and how the connection to combustion instability comes about are explained in the next paragraph. Moreover, in Chehroudi's work, in which an externally imposed acoustic field was used to simulate certain key aspects of their interaction in real engines, it was shown 
that at subcritical conditions the root mean square (RMS) fluctuation values of the dark-core length were much higher than those at near-critical and supercritical conditions by a factor of 4 to 6 at all velocity ratios; see Figures 23(b) and 24 (b). Also, as the outer-to-inner jet velocity ratio declined, the RMS value increased from 1-2 to values of about 7-8 inner-jet hole diameters at subcritical pressures.

Chehroudi [56] interpreted the RMS of the dense dark core as a reflection of mass fluctuations to a first-order approximation; combining it with measurements of a core dominant oscillation frequency consistent with the imposed acoustic field's resonant mode frequency, he then suggested that a connection to rocket combustion instability may be obtained from these data through examination of the RMS of the dark-core length fluctuations. Decreases in the darkcore length fluctuation levels (quantified through the RMS) were then interpreted as the reduced intrinsic sensitivity of the jet. Chehroudi [56] then stated the possibility that decreases in the dark-core length fluctuation levels could weaken a key feedback mechanism for the self-excitation process that is believed to drive the combustion instability in cryogenic LRE. This was offered as a possible explanation for the combustion stability improvements experienced in production engines under higher outer-to-inner jet velocity ratios (see also Figures 23 and 24). The effect of temperature ramping was linked to its impact on the outer-to-inner velocity ratio and hence was also explained. More details can be found in Davis and Chehroudi [63, 64], Davis [44], and Leyva et al. [65]. In other words, the dynamic behavior of the dark-core, specifically its axial length, was considered by Chehroudi [56] to be the culprit for coaxial jet injectors.

Chehroudi [56] has then compared some of his results (from nonreacting test rigs) with those taken in singleelement fired rocket engines and demonstrated consistency of results pertaining to the dark-core dynamics. For example, he noted that measured mean intact or dark-core length for SSME-like momentum flux ratios by Woodward et al. [66] in a $\mathrm{LOX} / \mathrm{GH}_{2}$-fired single-element rocket engine agreed with those of Davis and Chehroudi's $[63,64]$ nonreacting case. Additionally, Chehroudi [56] referred to the existence of the dark-core length fluctuations reported by Woodward et al. [66]. He also pointed at a recent work by Yang et al. [67] in which they performed tests in a fired single-element rocket equipped with a coaxial $\mathrm{LOX} / \mathrm{CH}_{4}$ injector. Their measurements of the dark core length indicated an increasing trend in the level of fluctuations when the outer-to-inner velocity ratio was decreased and the core oscillation spectra showed more high-frequency contents in jet oscillation at lower velocity ratios. Finally, Chehroudi [56], by referring to the work of Smith et al. [68] where subcritical-tosupercritical sweeps were carefully conducted in a fired rocket engine, indicated that very low RMS values of the darkcore length at near- and super-critical conditions and high RMS values at subcritical pressures, both measured by Davis and Chehroudi $[63,64]$ in their nonreacting experimental setup, were consistent with the fired-engine experimental observations by Smith et al. [68]. Hence, Smith et al. [68] 's reported unstable combustion behavior at subcritical pressures with high core unsteadiness correlates with Davis and Chehroudi's high RMS values at subcritical conditions, which was then interpreted as conditions leading to highly "sensitive" dark-core dynamic response to its surrounding. In short, Chehroudi indicated that these results in such fired engines were consistent with the Davis and Chehroudi $[63,64]$ conclusions cited above.

Leveraging the observation that some of the key findings in cold nonreacting jets were consistent with subscale fired rocket engine, all using coaxial injectors, Chehroudi [56] then attempted to employ these, particularly the single-jet's, information to offer a compelling argument in addressing the combustion instability in engines using impinging-jet injector designs. Whereas RMS of the core fluctuations was a key factor for coaxial injectors, Chehroudi [56] proposed that both the mean and RMS of the dark core fluctuations in each jet of an impinging-jet injector were key parameters.

On the engine side, Chehroudi [56] used the information that when the so-called stability parameter $(d n / V)$ was reduced in production engines possessing impinging-jet injectors, combustion instability emerged at some point within this change. This stable-to-unstable transition boundary has been discussed in details by Anderson et al. [57] and is referred to as the Hewitt correlation. To offer a possible explanation, Chehroudi [56] had to propose a hypothesis (explained in the next paragraph) which although intuitively acceptable, requires further verification. However, he, by using data from impinging-jet injector designs in production engines and the dark-core lengths measured from single jets reported here (Figure 9), was able to provide some initial support for the hypothesis. Next, the hypothesis itself and some of his supporting information are explained.

For example, Chehroudi [56] showed that a reduction in the $d n / V$ (stability parameter) through changes in either $d n$ or $V$ leads to shortening of the mean dark-core (or break-up) length for each jet in an impinging jet injector. He then stated that it was quite possible that as $d n / V$ was reduced in an engine, the mean dark core length reached a critical value $\left(L_{C, P \text { th }}\right)$ where one intuitively expects inherently high sensitivity (high RMS) for an impinging-jet injector "system" to its environmental acoustic field. Here, Chehroudi [56] hypothesized that the Hewitt stable-to-unstable transition point (or line), which is usually observed as $d n / V$ is reduced, was at or near a condition where the distance from the holes exit plane of the impinging injector to the impinging point (i.e., preimpingement length) reached a critical value $\left(L_{C, P \text { th }}\right)$, creating a situation somewhat similar to what is shown in Figure 31. The arrangement shown in this figure was considered to be most sensitive to the ambient acoustic field. Chehroudi [56] also demonstrated that a situation shown in Figure 31 could easily take place in the thrust chamber of production engines during operation considering typical geometrical dimensions of impinging jet injectors for these engines. He also provided experimental evidence by Anderson et al. [57] (Figures 28 and 29) from tests of nonreacting impinging jets conducted at elevated chamber pressures suggesting high level of sensitivity for the arrangement shown in Figure 31.

Considering what was discussed for the coaxial jet injector, one implication of the hypothesis Chehroudi proposed is that an impinging jet injector engine should be more 


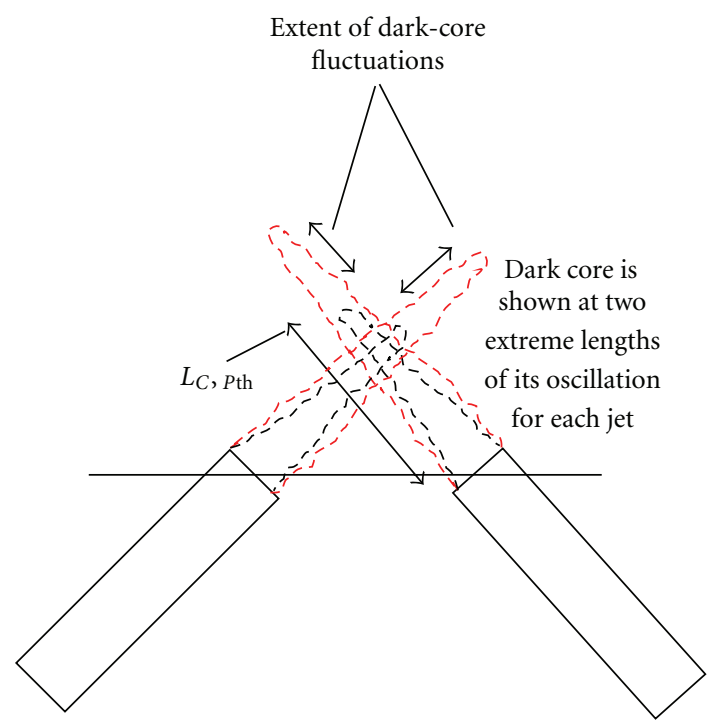

Figure 31: This figure shows the extent of the dark-core (or breakup) length fluctuations for individual jets of an impinging injector design for a situation when the average length $\left(L_{C, P \text { th }}\right)$ is of the same order as (or less than) the distance from each hole to the impinging point. Chehroudi [56].

stable at sufficiently high pressures, such as supercritical conditions. This is because not only the RMS of the core length fluctuations declines substantially (see Figures 23(b) and 24(b)), but also the length of the core may become adequately shorter than the pre-impingement length depending on the geometrical dimensions of the impinger. The changes in the dark-core (break-up) length can also be inferred by examination of Figure 30, showing a progressive increase in chamber pressure up to a supercritical condition for liquid nitrogen injection into gaseous nitrogen environment with no externally imposed acoustic field. The long preimpingement length seen along the jet is expected due to $L / d n$ of about 100 which was intentionally designed to obtain a fully developed condition at the hole exit plane and also to accentuate the effects of chamber pressure on the nature of the impingement. Obviously, shorter dark core is achieved for lower (injector hole) $L / d n$ values used in LRE. Not only the dark core length of each individual jet is reduced as supercritical pressures are approached (as before and expected), but the jet also becomes thicker. The impinger is expected to pass through a situation described in Figure 31 as chamber pressure is increased. Hypersensitivity is anticipated at that condition according to Chehroudi's hypothesis. Progressive increase of chamber pressure beyond this point sufficiently thickens each jet and shortens the darkcore length to a situation where the two dark-core lengths are shorter than the preimpingement distance and a gaslike jet is impinging another gas-like jet with enlarged crosssection areas. Based on Chehroudi's hypothesis and given that RMS of the dark core is much lower at supercritical than subcritical conditions, a more robust (targeting and mixing) and less sensitive impinging jet system would be expected at supercritical chamber pressures. However, it is likely that the dynamic behavior of the potential core plays a somewhat similar but weaker role under this latter gas-like condition.

Chehroudi's [56] attempt should be considered as the first step, but an important one, towards construction of a "big picture" through linkage of the increasing amount of experimental information made available. Essentially he was able to connect pieces of information from nonreacting test rigs, fired subscale engines, and production engines to provide an initial sketch. The sketch proposed by Chehroudi [56] has the advantage, simplicity, and the beauty as well, of identifying the possible weak link in the combustion stability assessment regardless of the design of the injector at least for the two popular cryogenic impinging and coaxial cases described. Much, of course, remains to be investigated, amongst them is the close examination of the historical data on the dynamic characteristics of the dark-core (or break-up) length and width for the circular jets forming the impinging injector for the propellant of interest and under the realistic thrust chamber conditions (which are quite rare or nonexistent) to further substantiate that a critical value, $L_{C, P \text { th }}$, is reached when the onset of instability is detected in an engine. Also, the dynamic characterization of each jet forming the impinging injector and when the two jets meet, in the presence of an externally imposed acoustic field, is highly desirable to assess sensitivity of the dark-core or break-up length of the jet to relevant design and operating variables.

\section{Conclusions}

It is evident from this relatively short and targeted review that much has been learned on jet breakup and relevant processes involved in cryogenic liquid propellant injection into highpressure supercritical condition over the past twenty years. In a quest to understand coaxial jet injectors used in $\mathrm{H}_{2} / \mathrm{O}_{2}$ cryogenic liquid rocket engines, systematic research efforts have been initiated which consider a logical progression from single jet injected into a quiescent environment to such jets under externally imposed acoustic field and to coaxial injectors with and without acoustic forcing, all under nonreacting conditions.

It is now clear that the impact of progressively lowered surface tension, as environmental pressure approaches the injectant's critical pressure from subcritical and exceeds to supercritical condition, suppresses classical liquid breakup and drop/ligament formation processes and a gas-like jet behavior emerges at or above the critical pressure of the injectant. It is also shown, quantitatively, and for the first time by Chehroudi's group that single liquid jets injected into a supercritical pressure and temperature behave similar to variable-density incompressible jets in visual growth rate. The gas-like nature of these single jets under such conditions has also been demonstrated through fractal analysis of the injected jet boundary. Based on physical arguments and visualizations, an effective model, and the only one known to this author, for the growth rate of such jets was proposed by Chehroudi, a model which mimics the experimental data quite well from sub- to supercritical chamber pressures and for different fluids. 
Visual length scales were measured which suggest conformance with the Taylor microscale. The Raman scattering measurements were conducted both for density profiles and growth rates. Based on this information, relationships between the same jet parameters when measured by shadowgraphy and by the Raman scattering were established. Similarity behavior in the radial density profile was demonstrated for the near- and super-critical single jets when the gas-like appearance emerged. Jet growth rates under supercritical pressures, as identified by density profiles, showed tendency to converge with those extrapolated for gaseous jets near the injector exit plane. Temperature estimates from the Raman data suggested existence of a pseudo-boiling behavior when the injection temperature is below the pseudo-boiling line at which the fluid-specific heat becomes very large (mathematical infinity). When the injected liquid possessing temperatures below the pseudo-boiling line warms up under supercritical chamber pressures, it eventually reaches this line, at which time most of the absorbed heat is taken up by the large specific heat value of the fluid, thus exhibiting a pseudo-boiling behavior.

Dark-core length dynamics of cryogenic single jets injected into both sub- and super-critical conditions were measured and compared with those of liquid fuel sprays and single-phase jets. At subcritical conditions, larger fluctuations of this length were reported than those under supercritical chamber pressures. Investigations on the response of the single jets to externally imposed acoustic waves indicated high sensitivity of the jet at subcritical pressures which then substantially diminished at supercritical pressures. Such a lack of response or jet's inherent insensitivity at supercritical pressures was explained through comparison with results extracted from similar studies conducted on single-phase (liquid and gaseous) jets.

Coaxial-jet studies revealed the existence of a dark core originating from the central hole exit area, similar to the single-jet case, but for the coaxial case it was surrounded by a cushion of the outer jet promoting liquid atomization at subcritical and mixing at supercritical pressures. The dark core interface assumed the appearance of a gaseous jet again under supercritical conditions similar to what was observed in a single-jet case. However, the possibility of delay in such a transition exists depending on the solubility of the inner jet and the critical mixing condition.

The mean dark-core length measurements in coaxial injectors with no externally imposed acoustic field exhibited a dual-character behavior depending on whether it experienced subcritical or supercritical chamber pressures. Results for the $\mathrm{LN}_{2}$ injection to $\mathrm{GN}_{2}$ were compared with a vast number of pertinent cases from single-phase gaseous and liquid flows to two-phase flows and found that the darkcore length scales with the outer-to-inner jet momentum flux ratio to the power of -0.2 , for the subcritical, and to -0.5 for supercritical pressures. Interestingly, as outer-toinner jet momentum flux ratio approaches zero (i.e., turning into a single jet), the dark-core length approaches values corresponding to the single-jet measurements. The growth rate for the coaxial jets, as measured by the visual spreading angle of the outer $\left(\mathrm{GN}_{2}\right)$ jet, agreed with that of supercritical pressures measured for the single jet but then declined below this value as one approached the supercritical pressures.

The impact of the external acoustic forcing on the coaxial jet was seen to be the highest at the subcritical pressures, similar to the single jet and substantially declined when reaching critical and supercritical chamber pressures. Effects of increases in outer-to-inner jet velocity ratio, an important design parameter for stable engine operation and recommended to be greater than 10 , were seen to dramatically reduce the mean dark-core length at subcritical pressures while having a minimal impact on this length under supercritical pressures. The dynamic behavior of the core was examined by measurements of the RMS of the core length fluctuations and interpreted as a factor influencing or contributing towards the propellant mass flow oscillations in a rocket thrust chamber. This too behaved very similar to the dark-core length mean value and was distinctively much higher at subcritical pressures than any others. This RMS behavior was then used to offer possible explanations for the empirical observation that stable engine operation required higher outer-to-inner jet velocity ratios (greater than about 10) and also for temperature ramping stability rating tests. Finally, for the injector used, the overall impact of the acoustic field was seen to be important only within a certain region of momentum flux ratios from about 1 to 5 .

Implications of a subset of these results were discussed in the context of the rocket combustion instability. Essentially, evidences were shown which suggest that the RMS of the dark-core length and its mean value are important for dynamic behavior of an impinging jet injector. It appears that for the coaxial jets, only RMS values are of relevance in this context.

A hypothesis was proposed that associates the stableto-unstable transition border in the Hewitt stability plot (for liquid rockets using impinging jets designs) to an event which is defined as an arrangement seen at a moment when the mean core length of one or more of the single jets in an impinging-jet injector design reaches the preimpingement distance. Some evidences were presented in support of this hypothesis. Essentially, a new alternate and compelling explanation for the Hewitt transition border was offered.

\section{References}

[1] T. J. Bruno and J. F. Ely, Supercritical Fluid Technology: Review in Modern Theory and Applications, CRC Press, 1991.

[2] R. S. Lazar and G. M. Faeth, "Bipropellant droplet combustion in the vicinity of the critical point," in Proceedings of the 30th Symposium (International) on Combustion, p. 801, The Combustion Institute, 1971.

[3] A. Umemura, "Supercritical droplet gasification combustion," in Proceedings of the IUTAM Symposium on Theories Combustion on Droplets and Sprays, Taiwan, December 1994.

[4] J. A. Newman and Brzustowski, "Behavior of a liquid jet near the thermodynamic critical region," AIAA Journal, vol. 9, no. 8, pp. 1595-1602, 1971.

[5] B. Chehroudi, D. Talley, and E. Coy, "Initial growth rate and visual characteristics of a round Jet into a sub- to supercritical 
environment of relevance to rocket, gas turbine, and diesel engines," in Proceedings of the 37th AIAA Aerospace Science Meeting and Exhibit, Reno, NV, USA, January 1999, AIAA 990206.

[6] W. Mayer, A. Schik, C. Schweitzer, and M. Schaffler, "Injection and mixing processes in high pressure LOX/GH2 rocket combustors," in Proceedings of the 32nd AIAA/ASME/SAE/ASEE Joint Propulsion Conference \& Exhibit, Lake Buena Vista, Fla, USA, 1996, AIAA Paper no. 96-2620.

[7] R. D. Reitz and F. B. Bracco, "On the dependence of spray angle and other spray parameters on nozzle design and operating condition," in Proceedings of the SAE International Congress and Exposition, Detroit, MI, USA, February-March 1979, SAE Paper no. 790494.

[8] W. Mayer, A. Ivancic, A. Schik, and U. Homung, "Propellant atomization in LOX/GH2 rocket combustors," in Proceedings of the 34th AIAA/ASME/SAE/ASEE Joint Propulsion Conference \& Exhibit, Cleveland, Ohio, USA, July 1998, Paper no. 983685.

[9] A. Roy and C. Segal, "Experimental study of subcritical to supercritical jet mixing," in Proceedings of the 47th AIAA ASM Meeting, Paper AIAA-2009-809, Orlando, Fla, USA, January 2008.

[10] B. Chehroudi, D. Talley, and E. Coy, "Visual characteristics and initial growth rates of round cryogenic jets at subcritical and supercritical pressures," Physics of Fluids, vol. 14, no. 2, pp. 850-861, 2002.

[11] C. J. Chen and W. Rodi, Vertical Turbulent Buoyant Jets: A Review of Experimental Data, Pergamon Press, 1980.

[12] P. N. Papanicolaou and E. J. List, "Investigations of round vertical turbulent buoyant jets," Journal of Fluid Mechanics, vol. 195, pp. 341-391, 1988.

[13] R. Branam and W. Mayer, "Characterization of cryogenic injection at supercritical pressure," Journal of Propulsion and Power, vol. 19, no. 3, pp. 342-355, 2003.

[14] G. N. Abramovich, The Theory of Turbulent Jets, MIT Press, Cambridge, UK, 1963.

[15] G. L. Brown and A. Roshko, "On density effects and large structure in turbulent mixing layers," Journal of Fluid Mechanics, vol. 64, no. 4, pp. 775-816, 1974.

[16] G. Brown, "The entrainment and large structure in turbulent mixing layers," in Proceedings of the 5th Australian Conference on Hydraulics and Fluid Mechanics, pp. 352-359, 1974.

[17] D. Papamoschou and A. Roshko, "The compressible turbulent shear layer: an experimental study," Journal of Fluid Mechanics, vol. 197, pp. 453-477, 1988.

[18] P. E. Dimotakis, "Two-dimensional shear-layer entrainment," AIAA Journal, vol. 24, no. 11, pp. 1791-1796, 1986.

[19] C. D. Richards and W. M. Pitts, "Global density effects on the self-preservation behaviour of turbulent free jets," Journal of Fluid Mechanics, vol. 254, pp. 417-435, 1993.

[20] B. Chehroudi, R. Cohn, D. Talley, and A. Badakhshan, "Raman scattering measurements in the initial region of sub- and supercritical jets," in Proceedings of the 36th Joint Propulsion Conference, Huntsville, AL, USA, 2000, AIAA 2000-3392.

[21] M. Oschwald and M. M. Micci, "Spreading angle and centerline variation of density of supercritical nitrogen jets," Atomization and Sprays, vol. 12, no. 1-3, pp. 91-106, 2002.

[22] B. B. Mandelbrot, The Fractal Geometry of Nature, W. H. Freeman and Company, San Francisco, Calif, USA, 1983.
[23] K. R. Sreenivasan and C. Meneveau, "The fractal facets of turbulence," Journal of Fluid Mechanics, vol. 173, pp. 357-386, 1986.

[24] J. J. Taylor and J. W. Hoyt, "Water jet photographytechniques and methods," Experiments in Fluids, vol. 1, no. 3, pp. 113-120, 1983.

[25] B. Chehroudi, D. Talley, and E. Coy, "Fractal geometry and growth rate of cryogenic jets near critical point," in Proceedings of the 35th AIAA/ASME/SAE/ASEE Joint Propulsion Conference, Los Angeles, Calif, USA, June 1999, AIAA Paper 99-2489.

[26] B. Chehroudi and D. Talley, "The fractal geometry of round turbulent cryogenic nitrogen jets at subcritical and supercritical pressures," Atomization and Sprays, vol. 14, no. 1, pp. 8191, 2004.

[27] B. Chehroudi, S.-H. Chen, F. V. Bracco, and Y. Onuma, "On the intact core of full-cone sprays," SAE Transaction 850126, 1985.

[28] M. Oschwald and A. Schik, "Supercritical nitrogen free jet investigated by spontaneous Raman scattering," Experiments in Fluids, vol. 27, no. 6, pp. 497-506, 1999.

[29] I. Wygnanski and H. E. Fiedler, "The two-dimensional Mixing region," Journal of Fluid Mechanics, vol. 41, no. 2, pp. 327-361, 1970.

[30] R. M. C. So, J. Y. Zhu, M. V. Ötügen, and B. C. Hwang, "Some measurements in a binary gas jet," Experiments in Fluids, vol. 9, no. 5, pp. 273-284, 1990.

[31] N. Zong, H. Meng, S.-Y. Hsieh, and V. Yang, "A numerical study of cryogenic fluid injection and mixing under supercritical conditions," Physics of Fluids, vol. 16, no. 12, pp. 42484261, 2004.

[32] M. Oschwald, A. Schik, M. Klar, and W. Mayer, "Investigation of coaxial $\mathrm{LN}_{2} / \mathrm{GH}_{2}$-injection at supercritical pressure by spontaneous raman scattering," in Proceedings of the 35th AIAA/ASME/SAE/ASEE Joint Propulsion Conference and Exhibit, Los Angeles, Calif, USA, June 1999.

[33] L.-K. Tseng, G. A. Ruff, P.-K. Wu, and G. M. Faeth, "Continuous- and dispersed-phase structure of pressureatomized sprays," in Proceedings of the Progress in Astronautics and Aeronautics: Recent Advances in Spray Combustion, 1995.

[34] N. Zong and V. Yang, "Cryogenic fluid jets and mixing layers in transcritical and supercritical environments," Combustion Science and Technology, vol. 178, no. 1-3, pp. 193-227, 2006.

[35] J. C. Oefelein and V. Yang, "Comprehensive review of liquidpropellant combustion instabilities in F-1 engines," Journal of Propulsion and Power, vol. 9, no. 5, pp. 657-677, 1993.

[36] B. Chehroudi and D. Talley, "Interaction of acoustic waves with a cryogenic nitrogen jet at sub- and supercritical pressures," in Proceedings of the 40th Aerospace Sciences Meeting and Exhibit, Reno, NV, USA, 2002, AIAA 2002-0342.

[37] D. O. Rockwell, "External excitation of planar jets," Journal of Applied Mechanics, vol. 39, no. 4, pp. 883-891, 1972.

[38] H. A. Becker and T. A. Massaro, "Vortex evolution in a round jet," Journal of Fluid Mechanics, vol. 31, pp. 435-448, 1968.

[39] Y. V. Vaslov and A. S. Ginevskiy, "Acoustic effects on aerodynamic characteristics of a turbulent jet," Tech. Rep. FTD-MT24-232-68, Foreign Technology Division, Air Force Systems Command, 1968.

[40] J. Hulka and J. J. Hutt, "Instability phenomena in liquid oxygen/hydrogen propellant rocket engines," in Liquid Rocket Engine Combustion Instability, V. Yang and W. E. Anderson, 
Eds., AIAA Progress in Astronautics and Aeronautics, pp. 3971, 1995.

[41] J. Telaar, G. Schneider, and W. Mayer, Experimental Investigation of Breakup of Turbulent Liquid Jets, ILASS-Europe 2000, Darmstadt, Germany, 2000.

[42] D. Davis and B. Chehroudi, "The effects of pressure and acoustic field on a cryogenic coaxial jet," in Proceedings of the 42nd AIAA Aerospace Sciences Meeting and Exhibit, pp. 1074110759, January 2004.

[43] D. Davis and B. Chehroudi, "Behaviour of a rocket-Like coaxial injector in an acoustic field, ILAS america," in Proceedings of the 19th Annual Conference on Liquid Atomization and Spray Systems, Toronto, Canada, May 2006.

[44] D. W. Davis, On the behavior of a shear-coaxial Jet, spanning sub- to super-critical pressures, with and without an externally imposed transverse acoustic field [Ph.D. thesis], Department of Mechanical and Nuclear Engineering, The Pennsylvania State University, 2006.

[45] M. Oschwald, J. J. Smith, R. Branam et al., "Injection of fluids into supercritical environments," Combustion Science and Technology, vol. 178, no. 1-3, pp. 49-100, 2006.

[46] M. Favre-Marinet and E. B. Camano Schettini, "Density field of coaxial jets with large velocity ratio and large density differences," International Journal of Heat and Mass Transfer, vol. 44, no. 10, pp. 1913-1924, 2001.

[47] H. Eroglu, N. Chigier, and Z. Farago, "Coaxial atomizer liquid intact lengths," Physics of Fluids A, vol. 3, no. 2, pp. 303-308, 1991.

[48] C. Englebert, Y. Hardalupas, and J. H. Whitlaw, "Article usage statistics center," Proceedings of the Royal Society A, vol. 451, pp. 189-229, 1995.

[49] Z. Faragó and N. Chigier, "Morphological classification of disintegration of round liquid jets in a coaxial air stream," Atomization and Sprays, vol. 2, pp. 137-153, 1992.

[50] R. D. Woodward, Primary atomization of liquid jets issuing from rocket engine coaxial Injectors [Ph.D. thesis], Pennsylvania State University, Department of Mechanical Engineering, University Park, Pa, USA, 1993.

[51] B. Chehroudi, D. Davis, and D. Talley, "Initial results from a cryogenic coaxial injector in an acoustic field," in Proceedings of the 41st Aerospace Sciences Meeting and Exhibit, Reno, NV, USA, 2003, AIAA 2003-1339.

[52] I. A. Leyva, J. I. Rodriguez, B. Chehroudi, and D. Talley, "Preliminary results on coaxial jet spread angles and the effects of variable phase transverse acoustic fields," in Proceedings of the 46th AIAA Aerospace Sciences Meeting and Exhibit, Reno, Nv, USA, January 2008.

[53] J. C. Lasheras and E. J. Hopfinger, "Liquid jet instability and atomization in a coaxial gas stream," Annual Review of Fluid Mechanics, vol. 32, pp. 275-308, 2000.

[54] J. I. Rodriguez, I. A. Leyva, B. Chehroudi, and D. Talley, in Proceedings of the 21st Annual Conference on Liquid Atomization and Spray Systems (ILASS '08), Orlando, Fla, USA, May 2008.

[55] J. I. Rodriguez, Acoustic excitation of liquid fuel droplets and coaxial jets [Ph.D. thesis], University of California at Los Angeles, 2009.

[56] B. Chehroudi, "Physical hypothesis for the combustion instability in cryogenic liquid rocket engines," Journal of Propulsion and Power, vol. 26, no. 6, pp. 1153-1160, 2010.
[57] W. E. Anderson, H. M. Ryan, R. J. Santoro, and R. A. Hewitt, "Combustion instability mechanisms in liquid rocket engines using impinging jet injectors," in Proceedings of the 31st AIAA/ASME/SAE/ASEE Joint Propulsion Conference and Exhibit, San Diego, Calif, USA, July 1995, Paper AIAA-952357.

[58] T. D. Harrje and H. F. Reardon, "Propellant rocket combustion instability," NASA Report, 1972, NASA SP-194.

[59] "Liquid rocket engine combustion instability", in Proceedings of the AIAA Progress in Astronautics and Aeronautics, V. Yang and W. E. Anderson, Eds., vol. 169, p. 577, 1995.

[60] L. Rayleigh, "The explanation of certain acoustical phenomena," in Proceedings of the Royal Institution, vol. 8, pp. 536-542, London, UK, 1878.

[61] M. F. Heidemann and J. F. Groeneweg, "Analysis of the dynamic response of liquid Jet atomization to acoustic oscillations," NASA Technical Note, 1969, NASA TN D-5339.

[62] T. Kiwata, A. Okajima, and H. Ueno, "Effects of excitation on plane and coaxial jets," in Proceedings of the 3rd Joint ASME/JSME Fluid Engineering Conference, pp. 18-22, San Francisco, Calif, USA, 1999.

[63] D. W. Davis and B. Chehroudi, "Measurements in an acoustically-driven coaxial jet under supercritical conditions," Journal of Propulsion and Power, vol. 23, no. 2, pp. 364-374, 2007.

[64] D. W. Davis and B. Chehroudi, "Shear-coaxial jets from a rocket-like injector in a transverse acoustic field at high pressures," in Proceedings of the 44th AIAA Aerospace Sciences Meeting, pp. 9173-9190, Reno, Nv, USA, January 2006, Paper No. AIAA-2006-0758.

[65] I. A. Leyva, B. Chehroudi, and D. Talley, "Dark core analysis of coaxial injectors at sub-, near-, and supercritical pressures in a transverse acoustic field," in Proceedings of the 43rd AIAA/ASME/SAE/ASEE Joint Propulsion Conference and Exhibit, pp. 4342-4359, Cincinnati, Ohio, USA, July 2007.

[66] R. D. Woodward, S. Pal, S. Farhangi, G. E. Jensen, and R. J. Santoro, "LOX/GH2 shear coaxial injector atomization studies: effect of recess and non-concentricity," in Proceedings of the 45th AIAA Aerospace Sciences Meeting and Exhibit, pp. 6925-6946, Reno, Nv, USA, January 2007.

[67] B. Yang, C. Francesco, L. Wang, and M. Oschwald, "Experimental investigation of reactive liquid Oxygen/CH4 coaxial sprays," Journal of Propulsion and Power, vol. 23, no. 4, pp. 763-771, 2007.

[68] J. J. Smith, M. Bechle, D. Suslov, M. Oschwald, O. J. Haidn, and G. M. Schneider, "High pressure LOx/H2 combustion and flame dynamics preliminary results," in Proceedings of the 40th AIAA/ASME/SAE/ASEE Joint Propulsion Conference \& Exhibit, Fort Lauderdale, Fla, USA, July 2004, AIAA-2004-3376. 

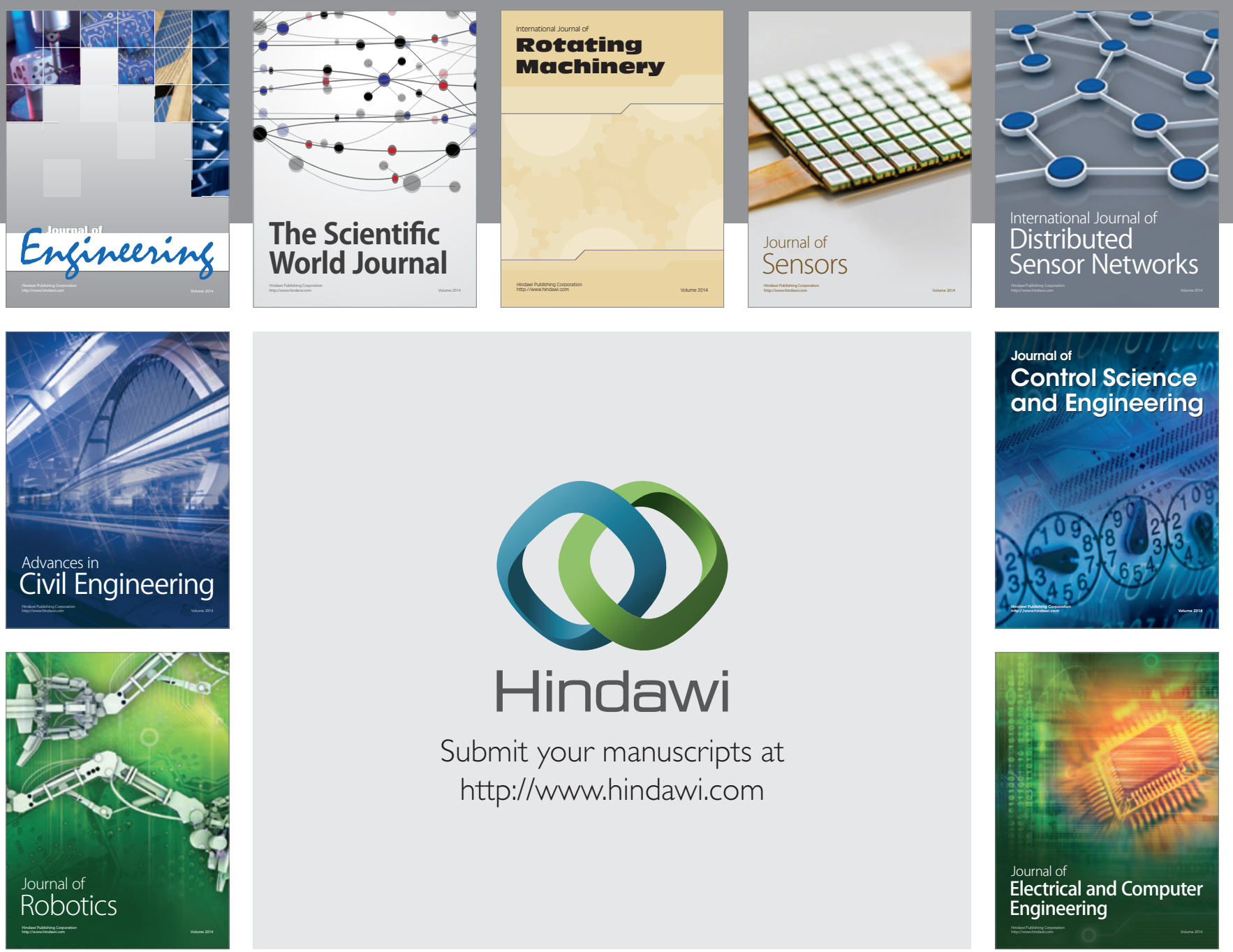

Submit your manuscripts at

http://www.hindawi.com
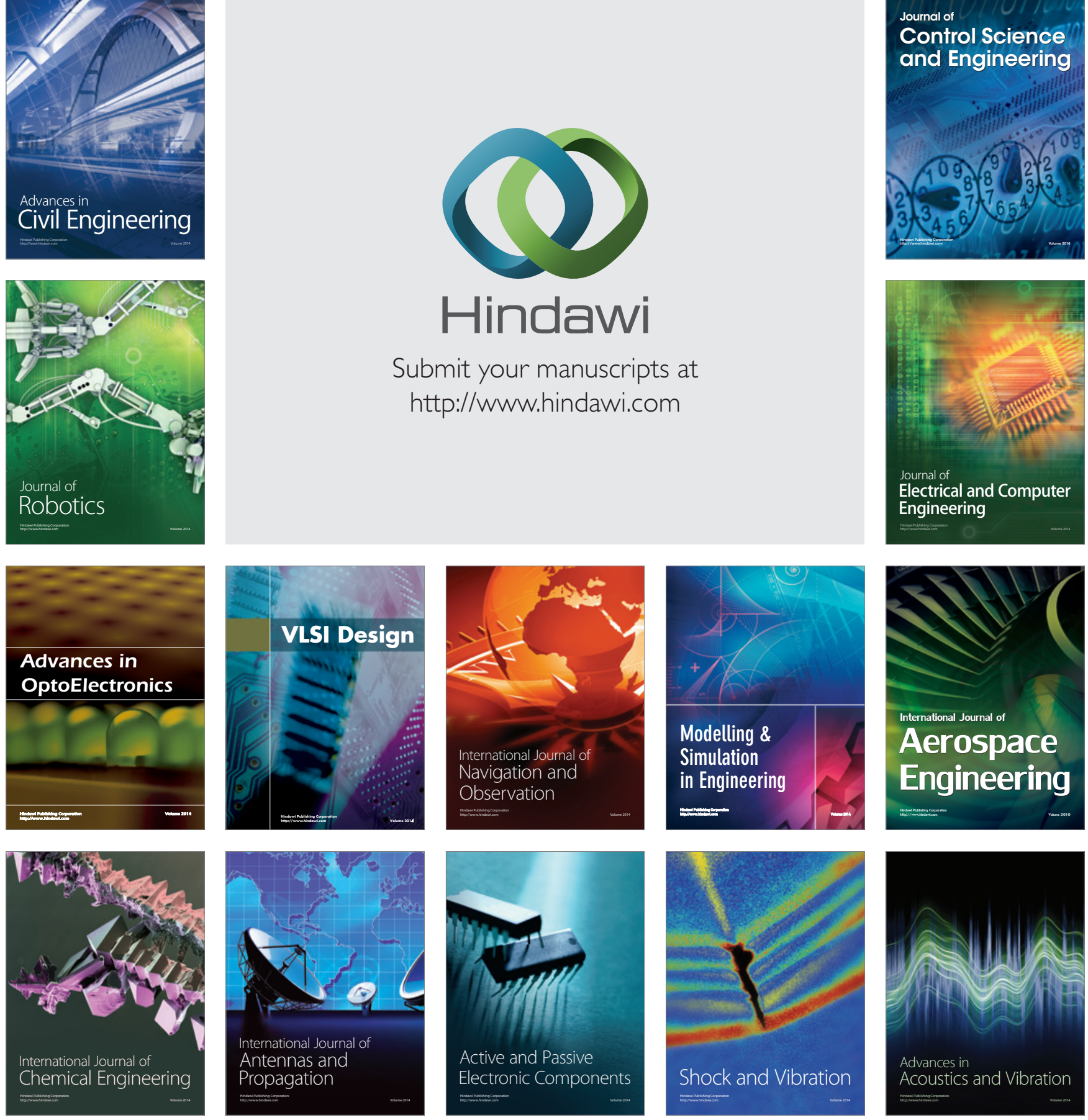